\title{
HYDROLOGIC DATA: NORTH CANADIAN RIVER FROM LAKE OVERHOLSER TO LAKE EUFAULA, CENTRAL OKLAHOMA
}

By John S. Havens

U.S. GEOLOGICAL SURVEY

Open-File Report 84-808

Prepared in cooperation with OKLAHOMA WATER RESOURCES BOARD

Oklahoma City, Oklahoma

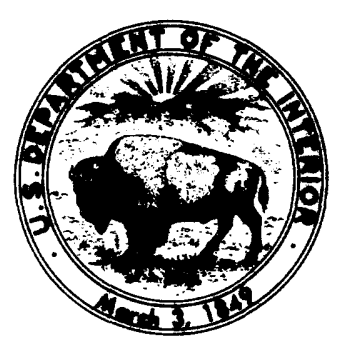

1984 


\section{UNITED STATES DEPARTMENT OF THE INTERIOR WILLIAM P. CLARK, Secretary}

\section{GEOLOGICAL SURVEY}

Dallas L. Peck, Director

For additional information write to:

District Chief

U.S. Geological Survey

Water Resources Division

Room 621, Oid Post Office Building

215 Dean A. McGee Avenue

Oklahoma City, Oklahoma 73102

Telephone: (405) 231-4256
Copies of this report can be purchased from:

Open-File Services Section

Western Distribution Branch

U.S. Geological Survey

Box 25425, Federal Center

Lakewood, Colorado 80225

Telephone: (303) 234-5888 


\section{CONTENTS}

Page

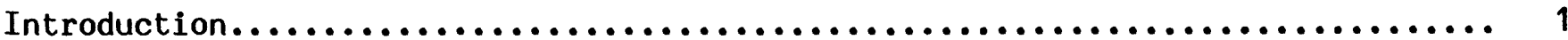

Explanation of the site-numbering system............................... 4

\section{ILLUSTRATIONS}

Figure 1.--Map showing locations of test holes and surface-water measuring and sampling sites.

Figure 2 - 15.--Hydrographs of wells, 1982-84:

2. Well 11N-05W-01 DCC 1, well 11N-04W-05 CDD 1, and well 11N-03W-05 BBC 135

3. Well 11N-03W-03 ADD 1, well 12N-02W-15 AAA 1, and well 13N-01W-18 CDD 136

4. Well 12N-01E-21 CBB 1, well 11N-02E-04 BAA 1, and well 10N-03E-06 DAA 137

5. Well 10N-03E-23 DCC 1, well 11N-04E-24 ABB 1, and well 11N-05E-21 DCD 138

6. Well 11N-05E-19 BAA 1, well 11N-07E-28 CCC 1, and well 11N-06E-27 CBC 139

7. Well 11N-06E-19 AAA 1, well 11N-07E-28 ABA 1, and well 11N-07E-33 CCD 140

8. Well 11N-08E-07 DDA 1, well 12N-08E-35 CBC 1, and well 11N-08E-12 BBB 141

9. Well 11N-08E-03 ADD 1, well 10N-09E-06 BAA 1, and well 10N-09E-15 BRB 142

10. Well 10N-09E-05 AAA 1, well 10N-09E-24 BBB 1, and well 10N-09E-13 DDD 143

11. Well 10N-10E-10 CCC 1, well 10N-11E-18 DCC 1, and well 09N-11E-08 DDD 144

12. Well 09N-10E-11 CAA 1, well 09N-11E-18 BBB 1, and well 10N-11E-14 DAD 145

13. Well 10N-12E-24 CCC 1, well 11N-13E-36 BDD 1, and well 11N-14E-26 ABB 146

14. Well 08N-10E-04 DAA 1, well 08N-09E-09 DDD 1, and well 08N-09E-18 CDA 147

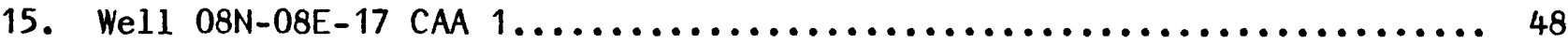


Figure 16.--Hydrographs of well 12N-01E-21 CBB 1 and North Canadian River near Harrah, 1983-84.

Figure 17.--Hydrographs of well 10N-12E-24 CCC 1 and North Canadian River near Wetumka, 1983-84

\section{TABLES}

Table 1.--Records of wells and test holes........................... 5

Table 2.--Field water-quality data for wells and test holes............... 15

Table 3.--Lithologic logs of test holes............................ 22

Table 4.--Low-flow measurements of the North Candian River and tributaries, $1983-84 \ldots \ldots \ldots \ldots \ldots \ldots \ldots \ldots \ldots \ldots \ldots \ldots \ldots \ldots \ldots$

Table 5.--Chemical analyses of water samples collected in conjunction with low-flow measurements of the North Canadian River and tributaries, $1983-84 \ldots \ldots \ldots \ldots \ldots \ldots \ldots \ldots \ldots \ldots \ldots \ldots \ldots \ldots \ldots \ldots \ldots \ldots \ldots$ 
HYDROLOGIC DATA: NORTH CANADIAN RIVER FROM LAKE OVERHOLSER

TO LAKE EUFAULA, CENTRAL OKLAHOMA.

by John S. Havens

\section{Introduction}

The data contained in this report were gathered during the period 1982 to 1984 for use in constructing a digital model of the North Canadian River from Lake Overholser, in the western part of Oklahoma City, to Lake Eufaula, in eastern 0klahoma. Locations of test holes and sampling sites are shown in figure 1.

Information on well depths and water levels in table 1 was gathered in the summer of 1982. Some information in the table was reported by well owners.

Field water-quality data for water temperatures, specific conductance, and $\mathrm{pH}$ were measured at the time the wells were inventoried in 1982 and appear in table 2.

Forty-nine test holes were augered to provide more comprehensive lithologic and water-level data along the North Canadian River. Lithologic logs of these test holes appear in table 3. Thirty-eight of the test holes were completed as observation wells by placing perforated plastic casing in the holes. Water levels were measured in these observation wells from the time of completion in mid-1982 through mid-1984. Hydrographs of the observation wells are shown in figures 2 through 15 . The data are presented graphically for clarity. 


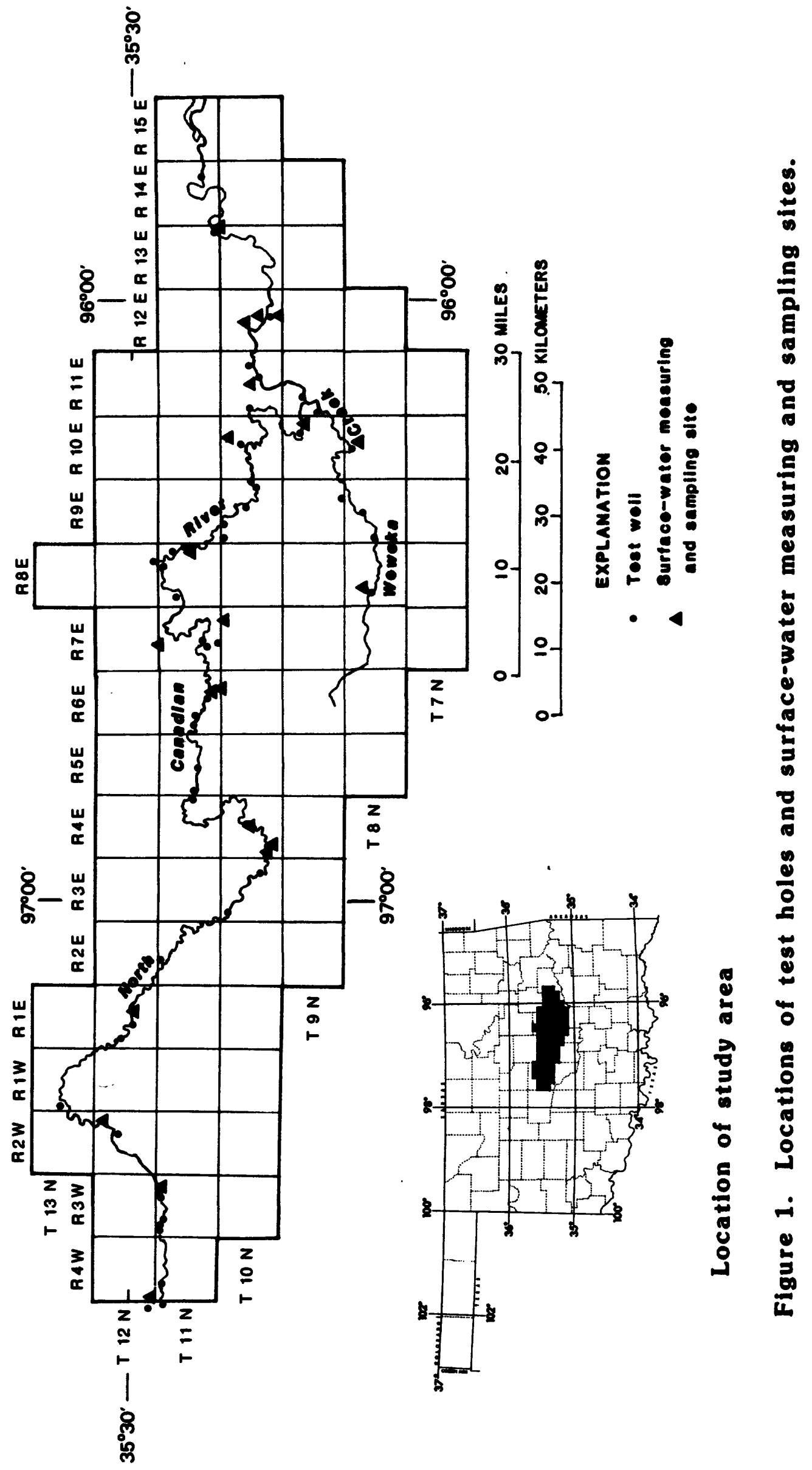


Hydrographs of water-level fluctuations in two wells equipped with continuous water-level recorders and hydrographs of stage fluctuations on the North Canadian River at nearby gaging stations are shown in figures 16 and 17.

Two sets of low-flow measurements for the North Canadian River showing gains and losses in flow between measuring sites in the reach from Lake Overholser to Lake Eufaula are given in table 4. Measurements of flow on tributary streams are also given in this table.

Analyses of water-quality samples collected at the time of the low-flow measurements are given in table 5 . 


\section{Explanation of the Site-Numbering System}

The standard legal method of describing locations of data-collection sites by fractional section, section, township, and range is replaced in this report by the method illustrated in the diagram below. By the legal method, the location of the site indicated by the dot is described as SW $1 / 4$ SW $1 / 4$ SE $1 / 4$ sec. 1, T.1l N., R. 5 W. The method used in this report indicates quarter subdivisions of the section by letters and reverses the order of presentation of the subdivisions. By this method, the location of the site is given as $11 \mathrm{~N}-05 \mathrm{~W}-01$ DCC 1 . The final digit (1) is the sequence number of the site within the smallest fractional subdivision. For example, if three data collection sites were located within the same quarter-quarter-quarter section, they would be uniquely identified as DCC 1, DCC 2 and DCC 3.

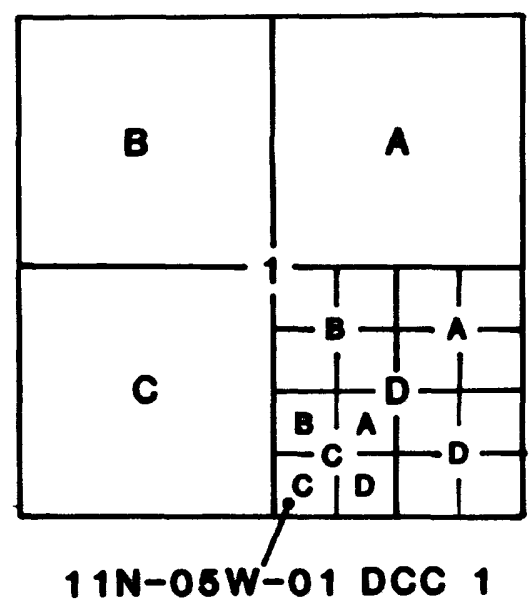


Table 1.--Records of wells and test holes.

Site-ID: Unique identification number that usually coincides with the latitude, longitude, and sequence number for the site.

Water level: Feet below land surface. $S$, measured with steel tape; $R$ or 0 , reported by owner.

Depth of well: Feet below land surface.

Use of water: $U$, unused; $H$, domestic; $S$, stock; $N$, industrial;

$I$, irrigation; $Z$, destroyed.

Other data avallable, QW: F, field data avallable; $A$, more complete water-quality data avallable.

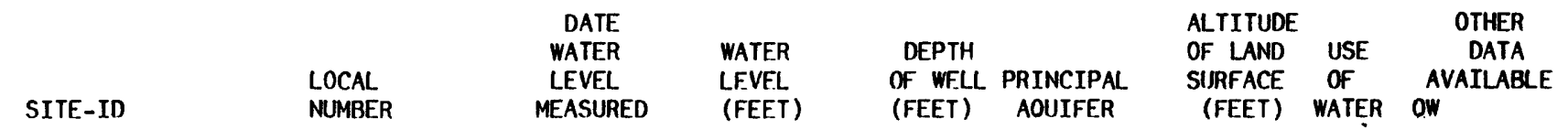

CANADIAN COUNTY

\begin{abstract}
352700096404501
352702097413301

352845097395201

352847097395302

352844097410601

352923097432501

352902097422701

352840097401901

352815097401901

352752097404601
\end{abstract}

HUGHES COUNTY
11N-O5W-01 DCC 1 $11 \mathrm{~N}-05 \mathrm{~W}-02$ DDC 1 $12 \mathrm{~N}-04 \mathrm{~W}-30$ CDD 1 $12 \mathrm{~N}-04 \mathrm{~W}-30 \mathrm{CDD} 2$ $12 \mathrm{~N}-05 \mathrm{~W}-25 \mathrm{CCD} 1$

12N-05W-27 BBC 1 12N-05W-27 DAD 1 12N-05W-36 AAA 1 12N-05W-36 DAA 12N-05W-36 DCC 1

08N-08E-23 CCD 1 $08 \mathrm{~N}-08 \mathrm{E}-25$ AAA 1 08N-08E-25 BAB 1 08N-09E-01 DAA 1 08N-09E-09 DDD 1

08N-09E-16 CCC 1 O8N-09E-17 ABA 1 08N-09E-18 CDA 1 08N-09E-19 ADD 1 08N-10E-04 ADB 1

08N-10E-04 DAA 1 09N-10E-11 CAA 1 09N-10E-11 CAB 1 09N-10E-13 BBB 1 09N-10E-24 DDD 1

\begin{abstract}
$06 / 28 / 1982$
$--$

$05 / 27 / 1982$

$05 / 27 / 1982$

$05 / 26 / 1982$

$05 / 28 / 1982$

$05 / 27 / 1982$

$05 / 26 / 1982$

$05 / 25 / 1982$
\end{abstract}
$23.70 \mathrm{~S}$
$3 . \overline{23}$
$3.23 \mathrm{~S}$
$11.84 \mathrm{~S}$
$21.05 \mathrm{~s}$
$0.85 \mathrm{~s}$
3.77 S
$15.80 \mathrm{~s}$
$20.00 \mathrm{R}$

38
--
33
16
62

$\begin{array}{ll}-- & 1230 \\ -- & 1260 \\ -- & 1220 \\ -- & 1220 \\ -- & 1241 \\ -- & 1270 \\ -- & 1250 \\ -- & 1240 \\ -- & 1220 \\ -- & 1235\end{array}$

12
27
--
43
85

\begin{abstract}
$07 / 20 / 1982$
$07 / 20 /$

$07 / 20 / 1982$

$08 / 11 / 1982$

$07 / 20 / 1982$

$07 / 20 / 1982$

$08 / 13 / 1982$

$07 / 2 \overline{1 / 1982}$

08/10/1982

$08 / 05 / 1982$

--

$07 / 13 / 1982$

$01 / 01 / 1981$
\end{abstract}
$41 . \overline{90} \mathrm{~S}$
$3.50 \mathrm{R}$
$11.56 \mathrm{~S}$
$12.10 \mathrm{~S}$

--
188
16
83
17

$\begin{array}{ll}-- & 820 \\ -- & 815 \\ -- & 810 \\ -- & 755 \\ -- & 750\end{array}$

$28.64 \mathrm{~S}$

$27.62 \mathrm{~S}$

$14.50 \mathrm{~S}$

$5.40 \mathrm{~s}$
33
31
30

$-2$

--
--
--
--
--
--
--

22
14
18
--
46


Table 1.--Records of wells and test holes.--Continued.

SITE-ID

LOCAL

NUMBER

\section{DATF-}

WATER

LEVEL

MEASURED
WATER

L.EVFL

(FEET)
DEPTH

OF WELL PRINCIPAL

(FEET) AOIIFER

\begin{tabular}{|c|c|c|}
\hline $\begin{array}{l}\text { ALTITUDE } \\
\text { OF LAND } \\
\text { SURFACF. }\end{array}$ & USE & $\begin{array}{c}\text { OTHER } \\
\text { DATA } \\
\text { AVAILARLE }\end{array}$ \\
\hline
\end{tabular}

(FEET) WATER OW
HIIGHES COUNTY--CONTINUED

351357096132101 351350096120901 351304096142201 351224096124601 351538096080301

351514096085801 351449096095401 351538096113401 351450096104801 351407096111601

351304096104501

LINCOLN COUNTY

352847097080701 352752097080401 352823097072401 352814097065601

12N-02E-30 CDC 1 $12 \mathrm{~N}-02 \mathrm{E}-31$ CDC 1 12N-02E-32 BCB 12N-02E-32 CAA 1

MCINTOSH COUNTY

351958095541101 352234096474301 352420095472601 352410095482301 352418096470801

10N-13E-23 BAA 1 10N-14E-02 ABB 1 $11 \mathrm{~N}-14 \mathrm{E}-26$ ABB 1 11N-14E-27 AAD 1 $11 \mathrm{~N}-14 \mathrm{E}-36 \mathrm{BBB} 1$

OKFUSKEE COUNTY
352213096215401 352237096221301 352238096235001 352012096180101 352007096175901

352213096210801 351959096185801 351930096180101 352054096144601 351944096165701
09N-10E-24 DDD 1 N-10E-25 BAA $09 N-10 E-35$ DAD 1 O9N-11E-15 BAR 1

09N-11E-16 BDD 1 ONN 11 - 17 DCC $09 \mathrm{~N}-11 \mathrm{E}-18$ DDC 1 09N-11E-19 CDB 1

09N-11E-30 DDC

\section{$01 / 01 / 1981$ \\ $07 / 21 / 1982$ \\ $07 / 20 / 1982$ \\ $07 / 21 / 1982$ \\ --}

$07 / 14 / 1982$

$07 / 14 / 1982$

$08 / 05 / 1982$

$07 / 21 / 1982$

$07 / 21 / 1982$

$\begin{array}{rr}6.00 & \\ 13.40 & S \\ 26.95 & S \\ 8.10 & S \\ -- & \\ & \\ 35.24 & S \\ 15.77 & S \\ 6.60 & S \\ 12.75 & S \\ 20.35 & S\end{array}$

46$$
\begin{array}{r}
2 \\
37 \\
130
\end{array}
$$$$
130
$$$$
\text { -- }
$$

\section{6}

43

15

135

65 $\begin{array}{cccccccc}-- & -- & -- & -- & 1070 & H & F \\ -- & 200.00 & R & -- & -- & 1073 & H & F \\ -- & -- & & -- & -- & 1060 & H & F \\ 06 / 15 / 1982 & 1.70 & S & 33 & -- & 1060 & I & \end{array}$

$07 / 14 / 1982$ $07 / 15 / 1982$ $08 / 10 / 1982$ $06 / 01 / 1982$ $07 / 15 / 1982$
$6.01 \mathrm{~S}$

$15.60 \mathrm{~S}$

$18.05 \mathrm{~S}$

$32.00 \mathrm{R}$

$42.95 \mathrm{~S}$

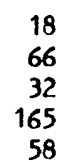

$--$
655

650

620

625

640
$F$
$F$
$F$
$F$
$F$

H

$\mathrm{H}$

H F
H F
10N-09E-04 BDC 1 10N-09E-05 AAA 1 10N-09E-06 BAA 1 10N-09E-13 DDA 1 10N-09E-13 DDD 1

10N-09E-15 BPB 1 10N-09E-24 RBB 1 10N-09E-24 DAA 1 10N-10E-10 CCC 1 1ON-10E-19 ADA 1
$07 / 07 / 1982$ $08 / 04 / 1982$ $08 / 04 / 1982$

$08 / 04 / 1982$

$08 / 03 / 1982$ $08 / 04 / 1982$ $07 / 08 / 1982$ $08 / 04 / 1982$ $07 / 08 / 1982$

$\begin{array}{cc}37.20 & \mathrm{~S} \\ 12.53 & \mathrm{~S} \\ 14.10 & \mathrm{~S} \\ -. & \\ 6.61 & \mathrm{~S} \\ & \\ 16.78 & \mathrm{~S} \\ 22.80 & \mathrm{~S} \\ 34.20 & \mathrm{~S} \\ 11.45 & \mathrm{~S} \\ 15.30 & \mathrm{~S}\end{array}$

58
18
32
--
15
21
30
98
25
23

$\begin{array}{ll}-- & 812 \\ -- & 785 \\ -- & 801 \\ -- & 771 \\ -- & 770 \\ & \\ -- & 781 \\ -- & 780 \\ -- & 795 \\ -- & 750 \\ -- & 750\end{array}$

$\begin{array}{cc}U & \\ -- & \\ -H & F \\ -- & \\ -- & \\ - & \\ - & F \\ -H & F\end{array}$


Table 1.--Records of wells and test holes.--Continued.

\begin{tabular}{|c|c|c|c|c|c|c|c|c|}
\hline SITE-ID & $\begin{array}{l}\text { LOC.AL } \\
\text { MUMBER }\end{array}$ & $\begin{array}{l}\text { DATE } \\
\text { WATER } \\
\text { LEVEL } \\
\text { MEASURED }\end{array}$ & $\begin{array}{l}\text { WATER } \\
\text { LEVEL } \\
\text { (FEET) }\end{array}$ & $\begin{array}{l}\text { DEPTH } \\
\text { OF WELL } \\
\text { (FEET) }\end{array}$ & $\begin{array}{l}\text { PRINCIPAL } \\
\text { AOUIFER }\end{array}$ & $\begin{array}{l}\text { ALTITUDE } \\
\text { OF LAND } \\
\text { SURFACE } \\
\text { (FEET) }\end{array}$ & $\begin{array}{l}\text { USE } \\
\text { OF } \\
\text { WATER }\end{array}$ & $\begin{array}{c}\text { OTHER } \\
\text { DATA } \\
\text { AVAILARLE } \\
\text { ON }\end{array}$ \\
\hline
\end{tabular}

OKFUSKEE COUNTY--CONTINUED

\begin{abstract}
352016096061901 352000096110401 351959096112501 351926096080401 351928096065101

351923096052801 352020096032701 351935096021801 351853096003401 351817096020301

352728096324701 352422096341201 352614096302101 352659096252301 352619096243701

352619096243701 352517096242001 352432096231901 352752096344701 352808096262701
\end{abstract}

10N-11E-14 DAD 1 $10 \mathrm{~N}-11 \mathrm{E}-18$ DCC 1 $10 \mathrm{~N}-11 \mathrm{E}-19$ BBA 1 10N-11E-22 CAC 1 1ON-11E-23 CAD 1

$10 \mathrm{~N}-11 \mathrm{~F}-24$ DAC 1 10N-12E-17 DBA 1 1ON-12E-21 ADC 1 10N-12E-26 BDA 1 $10 \mathrm{~N}-12 \mathrm{E}-27 \mathrm{CCr} 1$

11N-07E-02 RCC 1 $11 \mathrm{~N}-07 \mathrm{E}-28$ ABA 1 11N-08E-07 DDA 1 $11 \mathrm{~N}-08 \mathrm{E}-12$ BBB 1 $11 \mathrm{~N}-08 \mathrm{E}-12$ DCA 1

$11 \mathrm{~N}-08 \mathrm{E}-12$ DCA 1 $11 \mathrm{~N}-09 \mathrm{E}-18$ CCC 1 $11 \mathrm{~N}-09 \mathrm{E}-19$ DDA 1 12N-07E-33 CCD 1 $12 \mathrm{~N}-08 \mathrm{E}-35 \mathrm{CBC} 1$
$08 / 05 / 1982$

$08 / 04 / 1982$

$04 / 01 / 1982$

$07 / 13 / 1982$

$$
\text { -- }
$$

$07 / 14 / 1982$

$07 / 13 / 1982$

$07 / 14 / 1982$

$08 / 05 / 1982$

$07 / 06 / 1982$

$07 / 08 / 1982$

$08 / 02 / 1982$

$08 / 03 / 1982$

07/06/1982

$07 / 06 / 1982$

$07 / 06 / 1982$

$07 / 07 / 1982$

$08 / 02 / 1982$

08/03/1982
$16.04 \mathrm{~S}$

$18.13 \mathrm{~S}$

$13.75 \mathrm{R}$

$18.16 \mathrm{~S}$

--

$14.84 \mathrm{~S}$

$16.30 \mathrm{~s}$

$12.81 \mathrm{~s}$

$--$

$8.85 \mathrm{~S}$

$54.33 \mathrm{~S}$

$8.13 \mathrm{~S}$

$18.06 \mathrm{~S}$

$14.75 \mathrm{~S}$

$10.83 \mathrm{~S}$

$10.83 \mathrm{~S}$

$7.90 \mathrm{~S}$

$5.66 \mathrm{~S}$

$15.65 \mathrm{~S}$

0.0 s

$\begin{array}{rr}19 & - \\ 25 & - \\ 33 & - \\ 38 & -\end{array}$

--

37

119

54

$-\overline{16}$

$-$

$-$

$-$

$-$

$-$

$-$

$-$

$--$

$\overline{--}$

$--$

$-$
$69.65 \mathrm{~S} \quad 310$

$103.00 \mathrm{~S}$

$153.05 \mathrm{~S}$

$17.84 \mathrm{~S}$

$19.00 \mathrm{~S}$

$06 / 30 / 19$

$06 / 29 / 1982$

$06 / 01 / 1982$
--
$05 / 28 / 1982$

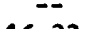

16.22

--

$11.00 \mathrm{~s}$

$\begin{array}{ccc}310 & -- & 1200 \\ 191 & -- & 1210 \\ 162 & -- & 1218 \\ 20 & -- & 1100 \\ 25 & -- & 1150 \\ & & \\ -- & -- & 1205 \\ 48 & -- & 1180 \\ -- & -- & - \\ -- & -- & 1225 \\ 53 & -- & 1220\end{array}$

\begin{tabular}{cc}
$H$ & F \\
H & F \\
$\mathbf{N}$ & $\mathbf{F}$ \\
\hdashline $\mathbf{U}$ & \\
& \\
$\mathbf{N}$ & $\mathbf{F}$ \\
$\mathbf{U}$ & $\mathbf{F}$ \\
$\mathbf{I}$ & $\mathbf{F}$ \\
$\mathbf{H}$ & $\mathbf{F}$ \\
$\mathbf{I}$ &
\end{tabular}


Table 1.--Records of wells and test holes.--Continuter.

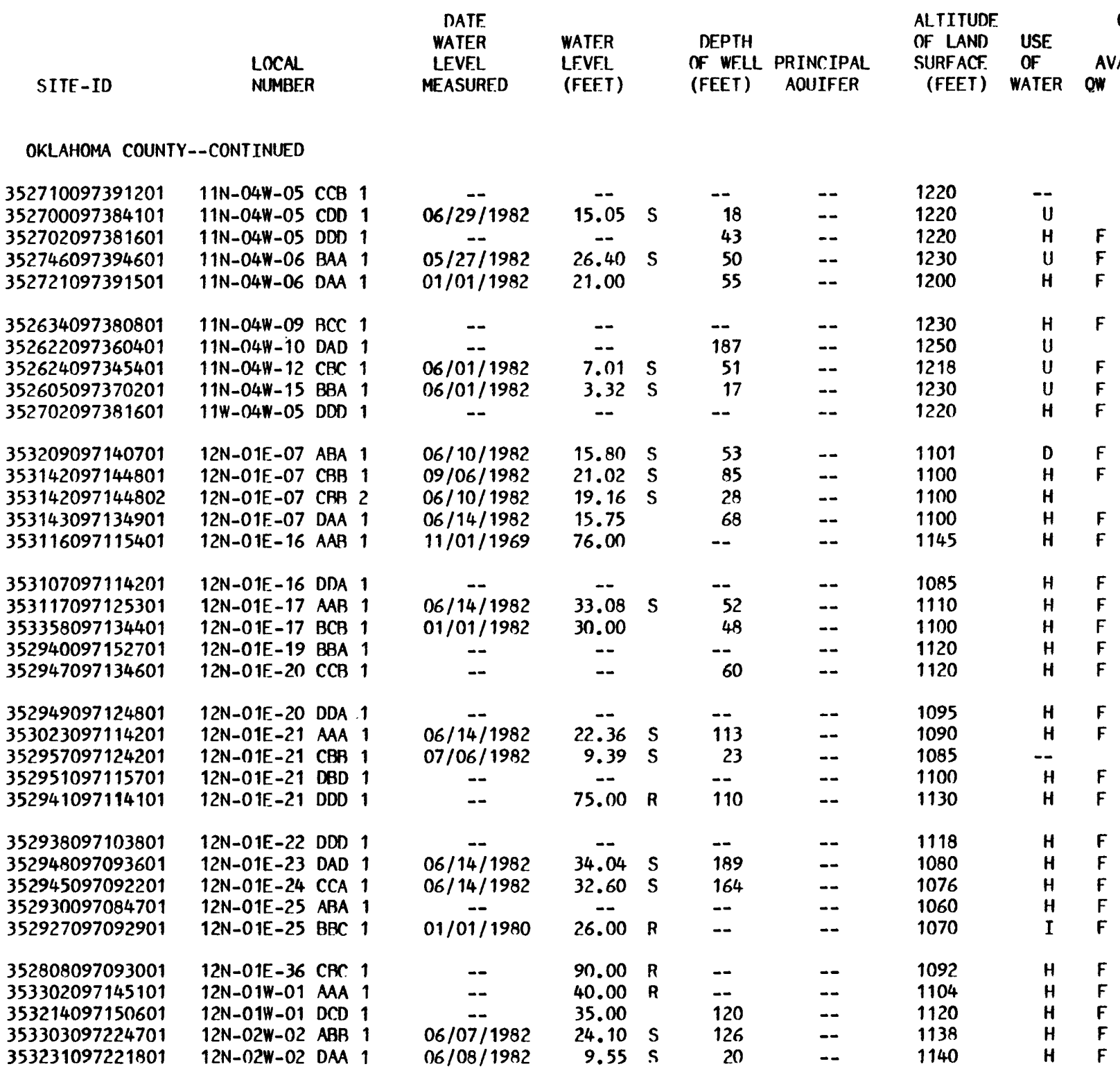


Table 1.--Records of wells and test holes.--Continued.

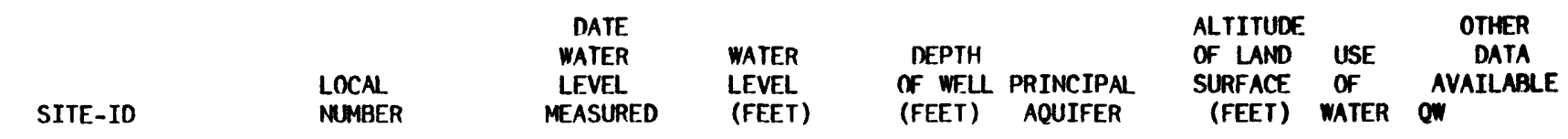

OKLAHOMA COUNTY--CONTINUED

\begin{tabular}{|c|c|c|c|c|c|c|c|c|c|c|}
\hline $\begin{array}{l}353214097224401 \\
353157097232101 \\
353210097240001 \\
353727097230701 \\
353138097231601\end{array}$ & $\begin{array}{l}12 N-02 W-02 \\
12 N-02 W-10 \\
12 N-02 W-10 \\
12 N-02 W-11 \\
12 N-02 W-11\end{array}$ & $\begin{array}{l}\text { DCC } 1 \\
\text { ADA } \\
\text { BAB } 1 \\
\text { BCA } \\
\text { CPC } 1\end{array}$ & $\begin{array}{l}1 \\
1 \\
1 \\
1 \\
1\end{array}$ & $\begin{array}{c}01 / 01 / 1970 \\
- \\
-- \\
06 / 07 / 1982 \\
06 / 07 / 1982\end{array}$ & $\begin{array}{c}8.38 \\
-- \\
-- \\
30.30 \\
23.65\end{array}$ & $\begin{array}{l}S \\
S\end{array}$ & $\begin{array}{r}146 \\
200 \\
85 \\
138 \\
106\end{array}$ & $\begin{array}{l}-- \\
-- \\
-- \\
--\end{array}$ & $\begin{array}{l}1138 \\
1140 \\
1180 \\
1150 \\
1144\end{array}$ & $\begin{array}{l}-- \\
H \\
H \\
H\end{array}$ \\
\hline $\begin{array}{l}353126097224001 \\
353154097221101 \\
353644097231901 \\
353054097232001 \\
353054097235901\end{array}$ & $\begin{array}{l}12 N-02 W-11 \\
12 N-02 W-12 \\
12 N-02 W-15 \\
12 N-02 W-15 \\
12 N-02 W-15\end{array}$ & $\begin{array}{l}\text { DCC } 1 \\
\text { BCB } \\
\text { AAA } 1 \\
\text { ADD } \\
\text { ADC } 1\end{array}$ & $\begin{array}{l}1 \\
1 \\
1 \\
1 \\
1\end{array}$ & $\begin{array}{c}06 / 08 / 1982 \\
06 / 08 / 1982 \\
07 / 01 / 1982 \\
-- \\
--\end{array}$ & $\begin{array}{c}29.28 \\
46.17 \\
16.60 \\
-. \\
--\end{array}$ & $\begin{array}{l}S \\
S \\
S\end{array}$ & $\begin{array}{l}36 \\
91 \\
20 \\
-- \\
--\end{array}$ & $\begin{array}{l}-- \\
-- \\
-- \\
-- \\
--\end{array}$ & $\begin{array}{l}1190 \\
1180 \\
1145 \\
1190 \\
1155\end{array}$ & $\begin{array}{c}\mathbf{U} \\
\mathbf{H} \\
-- \\
\mathbf{H} \\
\mathbf{H}\end{array}$ \\
\hline $\begin{array}{l}353147097251601 \\
353102097252901 \\
353024097261601 \\
352947097262701 \\
352939097254901\end{array}$ & $\begin{array}{l}12 N-02 W-16 \\
12 N-02 W-17 \\
12 N-02 W-20 \\
12 N-02 W-20 \\
12 N-02 W-20\end{array}$ & $\begin{array}{l}\text { BBB } \\
\text { DOD } \\
\text { BBA } 1 \\
\text { CCA } \\
\text { DCD }\end{array}$ & $\begin{array}{l}1 \\
1 \\
1 \\
1 \\
1\end{array}$ & $\begin{array}{c}06 / 04 / 1982 \\
- \\
06 / 04 / 1982 \\
-- \\
06 / 04 / 1982\end{array}$ & $\begin{array}{c}65.70 \\
-- \\
89.55 \\
-- \\
58.55\end{array}$ & $\begin{array}{l}\mathrm{S} \\
\mathrm{S} \\
\mathrm{S}\end{array}$ & $\begin{array}{l}96 \\
-- \\
158 \\
-- \\
158\end{array}$ & $\begin{array}{l}-- \\
-- \\
-- \\
--\end{array}$ & $\begin{array}{r}1210 \\
-- \\
1205 \\
1245 \\
1175\end{array}$ & $\begin{array}{l}H \\
H \\
H \\
H \\
H\end{array}$ \\
\hline $\begin{array}{l}352937097253001 \\
353017097245201 \\
353019097242101 \\
352855097252301 \\
352933097271701\end{array}$ & $\begin{array}{l}12 N-02 W-20 \\
12 N-02 W-21 \\
12 N-02 W-22 \\
12 N-02 W-28 \\
12 N-02 W-30\end{array}$ & $\begin{array}{l}\text { DDD } 1 \\
\text { ABC } \\
\text { BBC } 1 \\
\text { CCB } \\
\text { BAB }\end{array}$ & $\begin{array}{l}1 \\
1 \\
1 \\
1 \\
1\end{array}$ & $\begin{array}{c}06 / 04 / 1982 \\
06 / 09 / 1982 \\
-- \\
06 / 04 / 1982 \\
06 / 04 / 1982\end{array}$ & $\begin{array}{c}26.18 \\
24.00 \\
-- \\
17.80 \\
22.95\end{array}$ & $\begin{array}{l}\mathbf{S} \\
\mathbf{S} \\
\mathbf{R}\end{array}$ & $\begin{array}{r}107 \\
63 \\
-- \\
260 \\
160\end{array}$ & $\begin{array}{l}-- \\
-- \\
-- \\
--\end{array}$ & $\begin{array}{l}1160 \\
1160 \\
1150 \\
1170 \\
1245\end{array}$ & $\begin{array}{l}\text { U } \\
H \\
H \\
H \\
H\end{array}$ \\
\hline $\begin{array}{l}352934097272401 \\
352840097263201 \\
352815097272501 \\
352838097260101 \\
353253097391601\end{array}$ & $\begin{array}{l}12 N-02 W-30 \\
12 N-02 W-31 \\
12 N-02 W-31 \\
12 N-02 W-32 \\
12 N-04 W-06\end{array}$ & $\begin{array}{l}\text { BBA } 1 \\
\text { AAA } \\
\text { BCC } 1 \\
\text { BAD } 1 \\
\text { AAD } 1\end{array}$ & $\begin{array}{l}1 \\
1 \\
1 \\
1 \\
1\end{array}$ & $\begin{array}{c}-- \\
-- \\
-- \\
06 / 03 / 1982 \\
05 / 25 / 1982\end{array}$ & $\begin{array}{r}25.00 \\
150.00 \\
8.56 \\
64.70\end{array}$ & $\begin{array}{l}\mathbf{S} \\
\mathbf{S}\end{array}$ & $\begin{array}{r}- \\
150 \\
900 \\
100 \\
98\end{array}$ & $\begin{array}{l}-- \\
-- \\
-- \\
-- \\
--\end{array}$ & $\begin{array}{l}1240 \\
1130 \\
1160 \\
1160 \\
1279\end{array}$ & $\begin{array}{l}H \\
C \\
C \\
U \\
H\end{array}$ \\
\hline $\begin{array}{l}353145097394701 \\
352853097393101 \\
343000097394401 \\
352807097391701 \\
352816097390601\end{array}$ & $\begin{array}{l}12 N-04 W-07 \\
12 N-04 W-30 \\
12 N-04 W-30 \\
12 N-04 W-31 \\
12 N-04 W-32\end{array}$ & $\begin{array}{l}\text { CAA } 1 \\
\text { CAA } 1 \\
\text { DBC } 1 \\
\text { DAD } 1 \\
\text { CBA } 1\end{array}$ & $\begin{array}{l}1 \\
1 \\
1 \\
1 \\
1\end{array}$ & $\begin{array}{c}05 / 27 / 1972 \\
-- \\
05 / 27 / 1982 \\
-\end{array}$ & $\begin{array}{l}30.00 \\
25.00 \\
30.00 \\
14.55 \\
.-\end{array}$ & $\begin{array}{l}0 \\
\text { S }\end{array}$ & $\begin{array}{r}100 \\
45 \\
78 \\
54 \\
--\end{array}$ & $\begin{array}{l}-- \\
-- \\
-- \\
--\end{array}$ & $\begin{array}{l}1270 \\
1270 \\
1260 \\
1230 \\
1238\end{array}$ & $\begin{array}{l}H, S \\
H \\
H \\
U \\
H\end{array}$ \\
\hline $\begin{array}{l}352753097380501 \\
352821097360101 \\
353328097144001 \\
353606097175701 \\
353622097194501\end{array}$ & $\begin{array}{l}12 N-04 W-33 \\
12 N-04 W-35 \\
13 N-01 E-31 \\
13 N-01 W-15 \\
13 N-01 W-17\end{array}$ & $\begin{array}{ll}\text { CCD } & 1 \\
\text { BAC } & 1 \\
\text { CRA } & 1 \\
\text { CRB } & 1 \\
\text { BAC } & 1\end{array}$ & $\begin{array}{l}1 \\
1 \\
1 \\
1 \\
1\end{array}$ & $\begin{array}{c}01 / 06 / 1982 \\
06 / 08 / 1982 \\
--\end{array}$ & $\begin{array}{c}-- \\
15.00 \\
77 .-85 \\
--\end{array}$ & S & $\begin{array}{c}-- \\
-- \\
105 \\
163\end{array}$ & $\begin{array}{l}-- \\
-- \\
-- \\
--\end{array}$ & $\begin{array}{l}1230 \\
1220 \\
1100 \\
1150 \\
1180\end{array}$ & $\begin{array}{l}H \\
H \\
H \\
H \\
H\end{array}$ \\
\hline
\end{tabular}


Table 1.--Records of wells and test holes.--Continued.

\begin{tabular}{|c|c|c|c|c|c|c|c|c|}
\hline ITE-ID & $\begin{array}{l}\text { LOCAL } \\
\text { NIMMBER }\end{array}$ & $\begin{array}{l}\text { DATF } \\
\text { WATER } \\
\text { LEVEL } \\
\text { MEASURED }\end{array}$ & $\begin{array}{l}\text { WATER } \\
\text { LFVFL } \\
\text { (FEET) }\end{array}$ & $\begin{array}{l}\text { DEPTH } \\
\text { OF WFLL } \\
\text { (FEET) }\end{array}$ & $\begin{array}{c}\text { PRINCIPAL } \\
\text { AOUIFER }\end{array}$ & $\begin{array}{l}\text { ALTITUDE } \\
\text { OF LAND } \\
\text { SURFACF. } \\
\text { (FEET) }\end{array}$ & $\begin{array}{l}\text { USE } \\
\text { OF } \\
\text { WATER }\end{array}$ & $\begin{array}{c}\text { OTHER } \\
\text { DATA } \\
\text { AVAILABLE } \\
\text { OW }\end{array}$ \\
\hline
\end{tabular}

OKLAHOMA COUNTY--CONTINUED

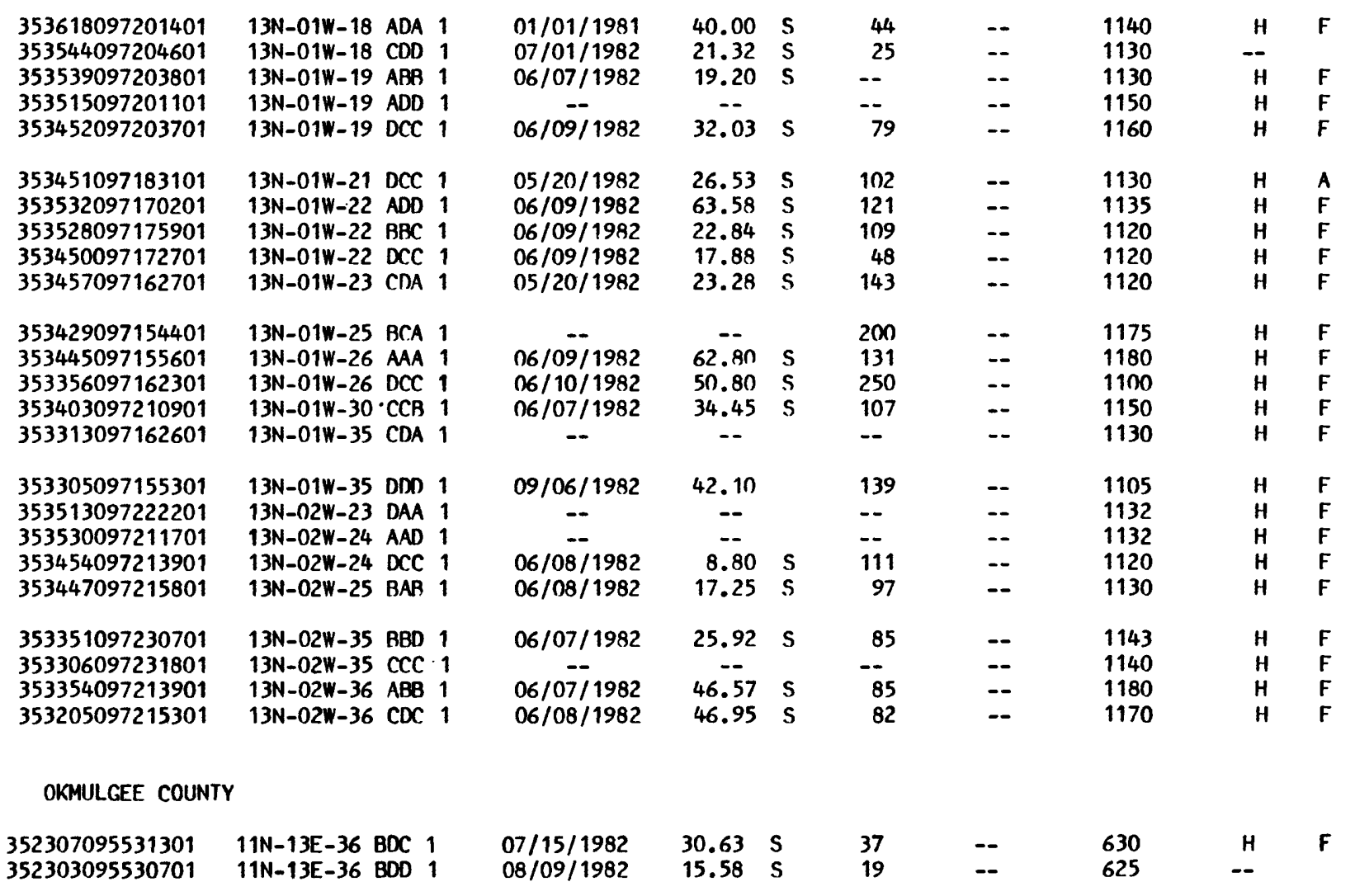


Table 1.--Records of wells and test holes.--Continued.

\begin{tabular}{|c|c|c|c|c|c|c|c|c|}
\hline TE-ID & $\begin{array}{l}\text { LOCAL } \\
\text { NUMRER }\end{array}$ & $\begin{array}{l}\text { DATE } \\
\text { WATFR } \\
\text { LFVFL } \\
\text { MEASURED }\end{array}$ & $\begin{array}{l}\text { WATFR } \\
\text { LFVFL } \\
\text { (FFFT) }\end{array}$ & $\begin{array}{l}\text { DFPTH } \\
\text { OF WFLL } \\
\text { (FEET) }\end{array}$ & $\begin{array}{l}\text { PRINCIPAL } \\
\text { AOUIFER }\end{array}$ & $\begin{array}{l}\text { ALTITIUDE } \\
\text { OF LAND } \\
\text { SURFACF } \\
\text { (FEET) }\end{array}$ & $\begin{array}{l}\text { USF } \\
\text { OF } \\
\text { WATER }\end{array}$ & $\begin{array}{c}\text { OTHER } \\
\text { DATA } \\
\text { AVAILARLE } \\
\text { ON }\end{array}$ \\
\hline
\end{tabular}

POTtamatomie COUNTY

\begin{abstract}
352227097010301
352155097000401

352219097020901

352208097010601

352145097010601

352139097000301

352141096585901

352141096595201

352059097000001

352056096585901

352140096581601 352006096574201 352046097575601 352000096594001 351909096580001

351956096565801 351936096571101 351915096572601 351923096565301 351908096572101

351907096561601 351819096561501 352330097021001 351817096573001 352152096493001

352220096513001 352110096502901 352139096502301 352001096501901 352000096511901

352026096523101 352003096515301 351959096523901 351910096553001 351920096540601
\end{abstract}

1ON-03E-05 BBC 1 10N-03E-05 DAA 1 10N-03E-06 BCB 1 10N-03E-06 DAA 1 10N-03E-06 DDD 1

10N-03E-08 AAD 1 10N-03E-09 AAA 1 10N-03E-09 RBA 1 10N-03E-09 CCB 1 10N-03E-09 DDD 1

1ON-03E-10 ARA 1 10N-03E-14 CCA 1 1ON-03E-15 AAA 1 10N-03E-16 CDC 1 10N-03E-22 DDD 1

10N-03E-23 AAA 1 10N-03E-23 ACD 1 1ON-03E-23 CDA 1 1ON-03E-23 DAD 1 1ON-03E-23 DCC 1

1ON-03E-24 DCC 1 10N-03E-25 CAC 1 10N-03E-25 DDD 1 $10 \mathrm{~N}-03 \mathrm{E}-26 \mathrm{CDD} 1$ 1ON-04E-01 DDB 1

10N-04E-02 BCB 1 10N-04E-11 DAA 1 $10 N-04 E-12$ BBR 1 $10 \mathrm{~N}-04 \mathrm{E}-13$ CCD 1 1ON-04E-14 CCD 1

10N-04E-15 BBC 1 10N-04E-15 DCD 1 $10 N-04 E-16$ DDD 1 $10 \mathrm{~N}-04 \mathrm{E}-19$ CDC 1 10N-04E-20 DRC 1
$06 / 18 / 1982$

$12 / 01 / 1981$

$07 / 07 / 1982$

$06 / 17 / 1982$

$01 / 01 / 1961$

$06 / 21 / 1982$

--

$06 / 18 / 1982$

$06 / 18 / 1982$

$40.51 \mathrm{~S}$

$20.00 \mathrm{R}$

$8.08 \mathrm{~S}$

$6.63 \mathrm{~S}$

94
90
--19
111

$16.00 \mathrm{R}$

$22.80 \mathrm{~S}$

$13.60 \mathrm{~s}$

8.675

60

58

$06 / 21 / 1982$

$06 / 22 / 1982$

$06 / 18 / 1982$

$$
\text { -. }
$$

16.99

$14.68 \mathrm{~S}$

$21.68 \mathrm{~S}$

-.

$05 / 01 / 1982$

$06 / 22 / 1982$

$$
\text { -- }
$$

$06 / 22 / 1982$

$07 / 07 / 1982$

$40.00 R$

$66.39 \mathrm{~S}$

--

$06 / 22 / 1982$

$06 / 22 / 1982$

$06 / 23 / 1982$

$37.58 \quad 5$

$4.87 \mathrm{~S}$

$13.32 \mathrm{~S}$

$19.60 \mathrm{~S}$

--

$06 / 23 / 1982$

$01 / 01 / 1980$

$01 / 01 / 1982$

$01 / 01 / 1977$

$01 / 01 / 1981$

$06 / 23 / 1982$

$06 / 23 / 1982$

$06 / 23 / 1982$

$06 / 23 / 1982$

$06 / 23 / 1982$

$20.85 \mathrm{~s}$

$30.00 \quad E$

$\begin{array}{ll}40.00 & R \\ 35.00 & Z\end{array}$

20.00

$20.00 \mathrm{R}$
$8.50 \mathrm{~S}$

$16.01 \mathrm{~S}$

$39.85 \mathrm{~S}$

$27.62 \mathrm{~S}$

$53 . \overline{78} \mathrm{~S}$

$--$

47

194

--

60

96

--

12

210

43

--

42

100

$--$

120

\begin{tabular}{|c|c|}
\hline -. & 1050 \\
\hline$=$ & 1040 \\
\hline-- & 1020 \\
\hline -- & 990 \\
\hline -- & $\cdots$ \\
\hline$\cdots$ & 1020 \\
\hline$\cdots$ & 1040 \\
\hline- & 1121 \\
\hline- & 1011 \\
\hline$\cdots$ & 1000 \\
\hline-- & 1039 \\
\hline -. & 1000 \\
\hline -- & 1010 \\
\hline -- & 1040 \\
\hline- & 1000 \\
\hline -- & 1050 \\
\hline-- & 1060 \\
\hline$\ldots$ & 995 \\
\hline- & 1050 \\
\hline-- & 995 \\
\hline-- & 1030 \\
\hline$\cdots$ & 1000 \\
\hline 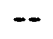 & 1020 \\
\hline- & 990 \\
\hline$-\infty$ & 960 \\
\hline$=-$ & 1010 \\
\hline- & 1008 \\
\hline 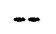 & 984 \\
\hline- & 1010 \\
\hline$\cdots$ & 1000 \\
\hline-- & 980 \\
\hline-- & 1010 \\
\hline- & 974 \\
\hline- & 1030 \\
\hline - & 1040 \\
\hline
\end{tabular}

68

39
47
72
31
64

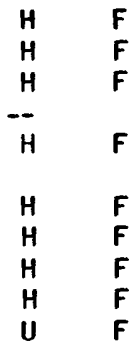

$\begin{array}{ll}H & \text { F } \\ \text { I } & \text { F } \\ \text { H } & \text { F } \\ \text { H } & \text { F } \\ \text { H } & \text { F }\end{array}$

1050

1060

1050

995

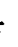

1030

1000

990

980
1010
974
1030
1040

$\begin{array}{ll}\text { U } & \text { F } \\ I, S & F \\ H & F \\ H & F \\ H & F \\ H & F \\ H & F \\ H & F \\ H & F \\ H & F \\ H & F \\ U & \\ H & F \\ H & F \\ H & F\end{array}$


Table 1.--Records of wells and test holes.--Continuer.

\begin{tabular}{|c|c|c|c|c|c|c|c|c|}
\hline & LOCAL & $\begin{array}{l}\text { DATE } \\
\text { WATER } \\
\text { LEVFL }\end{array}$ & $\begin{array}{l}\text { WATER } \\
\text { LEVEL }\end{array}$ & $\begin{array}{l}\text { DEPTH } \\
\text { OF WF.LL }\end{array}$ & PRINCIPAL & $\begin{array}{l}\text { ALTITUDF } \\
\text { OF LAND } \\
\text { SIIRFACE }\end{array}$ & $\begin{array}{l}\text { USE } \\
\text { OF }\end{array}$ & $\begin{array}{c}\text { OTHER } \\
\text { DATA } \\
\text { AVAILARLE }\end{array}$ \\
\hline SITE-ID & NUMBFR & MEASURFD & (FEET) & (FEFT) & AOUIFER & (FEET) & WATFR & OW \\
\hline
\end{tabular}

POTTAWATOMIE COUNTY--CONTINIFD

\begin{tabular}{|c|c|c|c|c|c|c|c|c|c|c|}
\hline $\begin{array}{l}351904096523401 \\
351905096533901 \\
351818096532701 \\
351818096541201 \\
351836096552801\end{array}$ & $\begin{array}{l}10 N-04 E-27 \\
10 N-04 E-28 \\
10 N-04 E-28 \\
10 N-04 E-29 \\
10 N-04 E-30\end{array}$ & $\begin{array}{ll}\text { BBA } & 1 \\
\text { BBB } & 1 \\
\text { CCD } & 1 \\
\text { DCC } & 1 \\
\text { CAB } & 1\end{array}$ & $\begin{array}{l}1 \\
1 \\
1 \\
1 \\
1\end{array}$ & $\begin{array}{l}06 / 23 / 1982 \\
06 / 23 / 1982 \\
05 / 01 / 1982 \\
06 / 23 / 1982 \\
06 / 22 / 1982\end{array}$ & $\begin{array}{r}5.38 \\
20.30 \\
10.00 \\
14.16 \\
6.25\end{array}$ & $\begin{array}{l}S \\
S \\
R \\
S \\
S\end{array}$ & $\begin{array}{r}97 \\
119 \\
184 \\
46 \\
35\end{array}$ & $\begin{array}{l}-- \\
-- \\
-- \\
-- \\
--\end{array}$ & $\begin{array}{l}990 \\
990 \\
995 \\
990 \\
980\end{array}$ & $\begin{array}{l}U \\
H \\
H \\
H \\
S\end{array}$ \\
\hline $\begin{array}{l}351753096545001 \\
352130096492401 \\
352104096492601 \\
352511097033101 \\
352748097055201\end{array}$ & $\begin{array}{l}10 N-04 E-31 \\
10 N-05 E-07 \\
10 N-05 E-07 \\
11 N-01 E-23 \\
11 N-02 E-04\end{array}$ & $\begin{array}{ll}A D D & 1 \\
\text { BCB } & 1 \\
\text { CCB } & 1 \\
\text { ABA } & 1 \\
\text { BAA } & 1\end{array}$ & $\begin{array}{l}1 \\
1 \\
1 \\
1 \\
1\end{array}$ & $\begin{array}{c}06 / 01 / 1973 \\
06 / 24 / 1982 \\
06 / 23 / 1982 \\
-- \\
07 / 06 / 1982\end{array}$ & $\begin{array}{l}25.00 \\
19.32 \\
16.10 \\
50.00 \\
10.32\end{array}$ & $\begin{array}{l}R \\
S \\
S \\
R \\
S\end{array}$ & $\begin{array}{c}-- \\
55 \\
41 \\
-- \\
22\end{array}$ & $\begin{array}{l}-- \\
-- \\
-- \\
--\end{array}$ & $\begin{array}{r}995 \\
970 \\
970 \\
1050 \\
1050\end{array}$ & $\begin{array}{c}H \\
U \\
I \\
H \\
--\end{array}$ \\
\hline $\begin{array}{l}352702097055401 \\
352718097071301 \\
352658097264501 \\
352647097062201 \\
352630097061501\end{array}$ & $\begin{array}{l}11 \mathrm{~N}-02 \mathrm{E}-04 \\
11 \mathrm{~N}-02 \mathrm{E}-05 \\
11 \mathrm{~N}-02 \mathrm{E}-05 \\
11 \mathrm{~N}-02 \mathrm{E}-09 \\
11 \mathrm{~N}-02 \mathrm{E}-09\end{array}$ & $\begin{array}{ll}\text { CDD } & 1 \\
\text { CBA } & 1 \\
D C D & 1 \\
B A C & 1 \\
\text { CBA } & 1\end{array}$ & $\begin{array}{l}1 \\
1 \\
1 \\
1 \\
1\end{array}$ & $\begin{array}{c}-- \\
06 / 15 / 1982 \\
-- \\
-\overline{06 / 16 / 1982}\end{array}$ & $\begin{array}{l}-- \\
44.55 \\
17.00 \\
45.00 \\
59.80\end{array}$ & $\begin{array}{l}S \\
R \\
R \\
S\end{array}$ & $\begin{array}{c}-- \\
95 \\
31 \\
-- \\
89\end{array}$ & $\begin{array}{l}-- \\
-- \\
-- \\
-- \\
--\end{array}$ & $\begin{array}{l}1050 \\
1085 \\
1085 \\
1095 \\
1128\end{array}$ & $\begin{array}{l}H \\
H \\
H \\
H \\
H\end{array}$ \\
\hline $\begin{array}{l}352656097042801 \\
352654097051301 \\
352642097034801 \\
352619097023001 \\
352619097032001\end{array}$ & $\begin{array}{l}11 \mathrm{~N}-02 \mathrm{E}-10 \\
11 \mathrm{~N}-02 \mathrm{E}-10 \\
11 \mathrm{~N}-02 \mathrm{E}-11 \\
11 \mathrm{~N}-02 \mathrm{E}-11 \\
11 \mathrm{~N}-02 \mathrm{E}-12\end{array}$ & 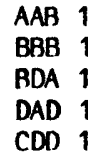 & $\begin{array}{l}1 \\
1 \\
1 \\
1 \\
1\end{array}$ & $\begin{array}{c}06 / 16 / 1982 \\
-- \\
06 / 16 / 1982 \\
06 / 16 / 1982 \\
--\end{array}$ & $\begin{array}{c}14.53 \\
\\
22.55 \\
18.78 \\
--\end{array}$ & $\begin{array}{l}\mathbf{S} \\
\mathbf{S}\end{array}$ & $\begin{array}{r}95 \\
125 \\
108 \\
56 \\
--\end{array}$ & $\begin{array}{l}-- \\
-- \\
-- \\
--\end{array}$ & $\begin{array}{l}1050 \\
1055 \\
1150 \\
1055 \\
1050\end{array}$ & $\begin{array}{l}H \\
H \\
H \\
H \\
H\end{array}$ \\
\hline $\begin{array}{l}352540097021601 \\
352540097043301 \\
352450097031301 \\
352330097023901 \\
352407097031801\end{array}$ & $\begin{array}{l}11 \mathrm{~N}-02 \mathrm{E}-13 \\
11 \mathrm{~N}-02 \mathrm{E}-15 \\
11 \mathrm{~N}-02 \mathrm{E}-24 \\
11 \mathrm{~N}-02 \mathrm{E}-25 \\
11 \mathrm{~N}-02 \mathrm{E}-26\end{array}$ & $\begin{array}{l}\text { ADD. } 1 \\
\text { ADC } 1 \\
\text { BCC } 1 \\
\text { DCC } 1 \\
\text { ADA } 1\end{array}$ & $\begin{array}{l}1 \\
1 \\
1 \\
1 \\
1\end{array}$ & $\begin{array}{c}-- \\
-- \\
06 / 17 / 1982 \\
06 / 17 / 1982 \\
06 / 16 / 1982\end{array}$ & $\begin{array}{l}15.00 \\
25.35 \\
42.70 \\
18.80\end{array}$ & $\begin{array}{l}R \\
S \\
S \\
S\end{array}$ & $\begin{array}{r}-- \\
-- \\
103 \\
98 \\
108\end{array}$ & $\begin{array}{l}-- \\
-- \\
-- \\
--\end{array}$ & $\begin{array}{l}1065 \\
1065 \\
1045 \\
1032 \\
1040\end{array}$ & $\begin{array}{l}H \\
H \\
H \\
H \\
H\end{array}$ \\
\hline $\begin{array}{l}350238097230401 \\
352233097010301 \\
352459097020601 \\
352419097015301 \\
352330097014601\end{array}$ & $\begin{array}{l}11 \mathrm{~N}-02 E-36 \\
11 \mathrm{~N}-03 \mathrm{E}-05 \\
11 \mathrm{~N}-03 \mathrm{E}-19 \\
11 \mathrm{~N}-03 \mathrm{E}-30 \\
11 \mathrm{~N}-03 \mathrm{E}-30\end{array}$ & $\begin{array}{l}\text { ACC } 1 \\
\text { BBB } 1 \\
\text { BCC } 1 \\
\text { BBA } 1 \\
\text { CDC } 1\end{array}$ & $\begin{array}{l}1 \\
1 \\
1 \\
1 \\
1\end{array}$ & $\begin{array}{c}-- \\
06 / 17 / 1982 \\
06 / 16 / 1982 \\
06 / 17 / 1982 \\
--\end{array}$ & $\begin{array}{c}-- \\
45.58 \\
26.15 \\
14.38 \\
--\end{array}$ & $\begin{array}{l}S \\
S \\
S\end{array}$ & $\begin{array}{r}100 \\
80 \\
57 \\
72 \\
--\end{array}$ & $\begin{array}{l}-- \\
-- \\
-- \\
-- \\
--\end{array}$ & $\begin{array}{l}1033 \\
1030 \\
1042 \\
1040 \\
1030\end{array}$ & $\begin{array}{l}H \\
H \\
H \\
H \\
H\end{array}$ \\
\hline $\begin{array}{l}352313097011901 \\
352302097010601 \\
352513096502701 \\
352603096494001 \\
352600096205501\end{array}$ & $\begin{array}{l}11 N-03 E-31 \\
11 N-03 E-31 \\
11 N-04 E-13 \\
11 N-04 E-14 \\
11 N-04 E-14\end{array}$ & $\begin{array}{l}\text { ADR } 1 \\
\text { ADD } 1 \\
\text { CCC } 1 \\
\text { AAB } 1 \\
\text { ABR } 1\end{array}$ & $\begin{array}{l}1 \\
1 \\
1 \\
1 \\
1\end{array}$ & $\begin{array}{c}\overline{--} \\
06 / 17 / 1982 \\
-- \\
-\overline{-} \\
06 / 24 / 1982\end{array}$ & $\begin{array}{c}-\overline{82} \\
21.82 \\
-\overline{-} \\
41.09\end{array}$ & $\mathbf{S}$ & $\begin{array}{r}-- \\
-- \\
50 \\
100 \\
67\end{array}$ & $\begin{array}{l}-- \\
-- \\
-- \\
-- \\
--\end{array}$ & $\begin{array}{r}1050 \\
1045 \\
930 \\
990 \\
970\end{array}$ & $\begin{array}{l}H \\
H \\
H \\
H \\
H\end{array}$ \\
\hline
\end{tabular}


Tahle 1.--Records of wells and test holes.--Continued.

\begin{tabular}{|c|c|c|c|c|c|c|c|c|}
\hline [TE-ID & $\begin{array}{l}\text { LOCAL } \\
\text { NUMBER }\end{array}$ & $\begin{array}{l}\text { DATE } \\
\text { WATER } \\
\text { LEVFL } \\
\text { MEASURED }\end{array}$ & $\begin{array}{l}\text { WATER } \\
\text { LFVFL } \\
\text { (FEET) }\end{array}$ & $\begin{array}{l}\text { DEPTH } \\
\text { OF WFLL } \\
\text { (FEET) }\end{array}$ & $\begin{array}{l}\text { PRINCIPAL } \\
\text { AOUIFER }\end{array}$ & $\begin{array}{l}\text { ALTITUDE } \\
\text { OF LAND } \\
\text { SURFACE } \\
\text { (FEET) }\end{array}$ & $\begin{array}{l}\text { USE: } \\
\text { OF } \\
\text { WATER }\end{array}$ & $\begin{array}{c}\text { OTHER } \\
\text { DATA } \\
\text { AVAILABLE } \\
\text { OW }\end{array}$ \\
\hline
\end{tabular}

POTTAWATOMIE COUNTY--CONTINUED

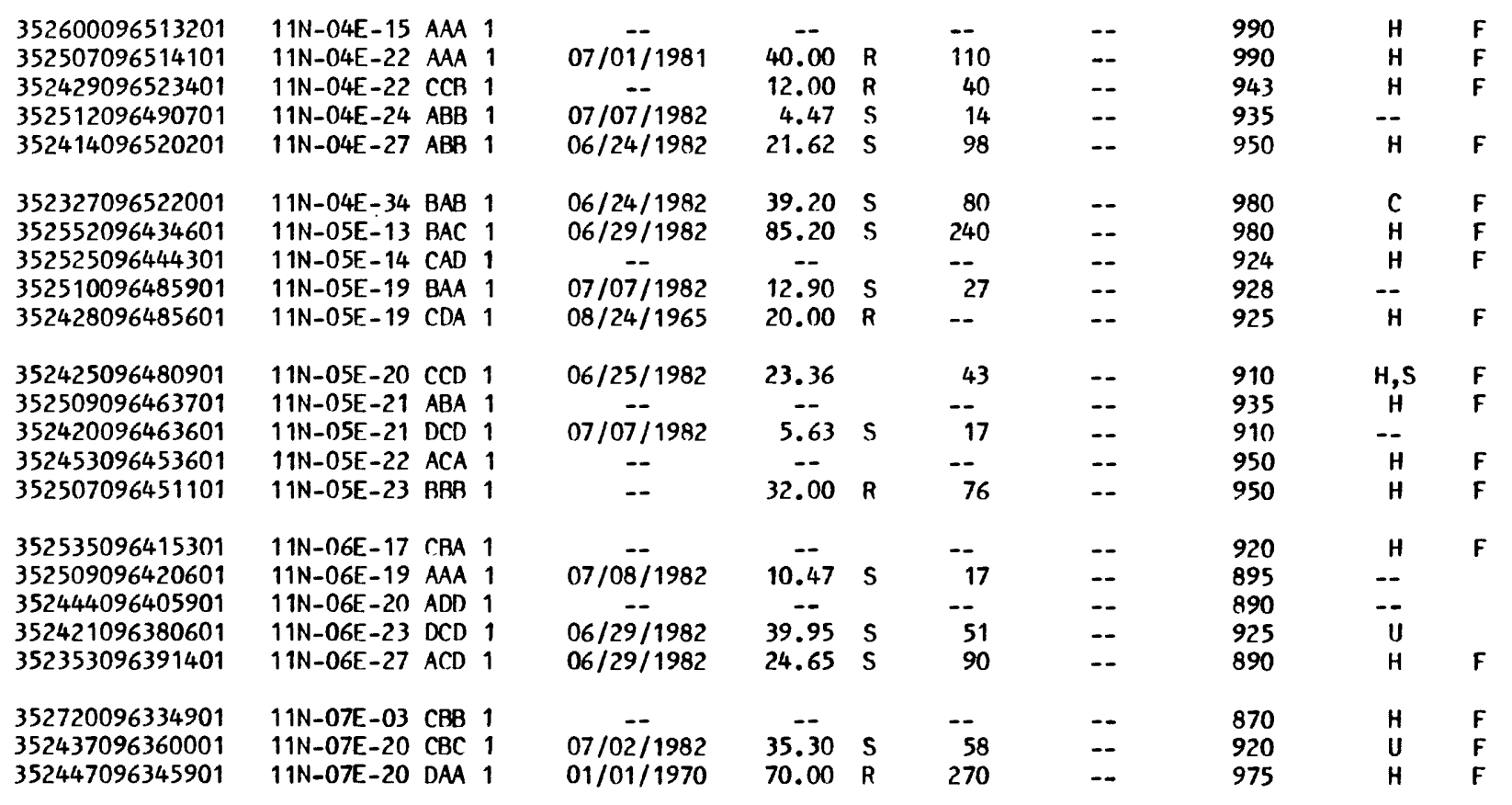


Table 1.--Records of wells and test holes.--Continued.

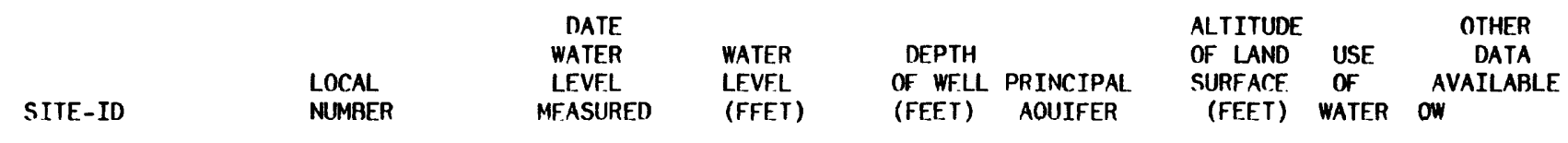

SEMINOLF COUNTY

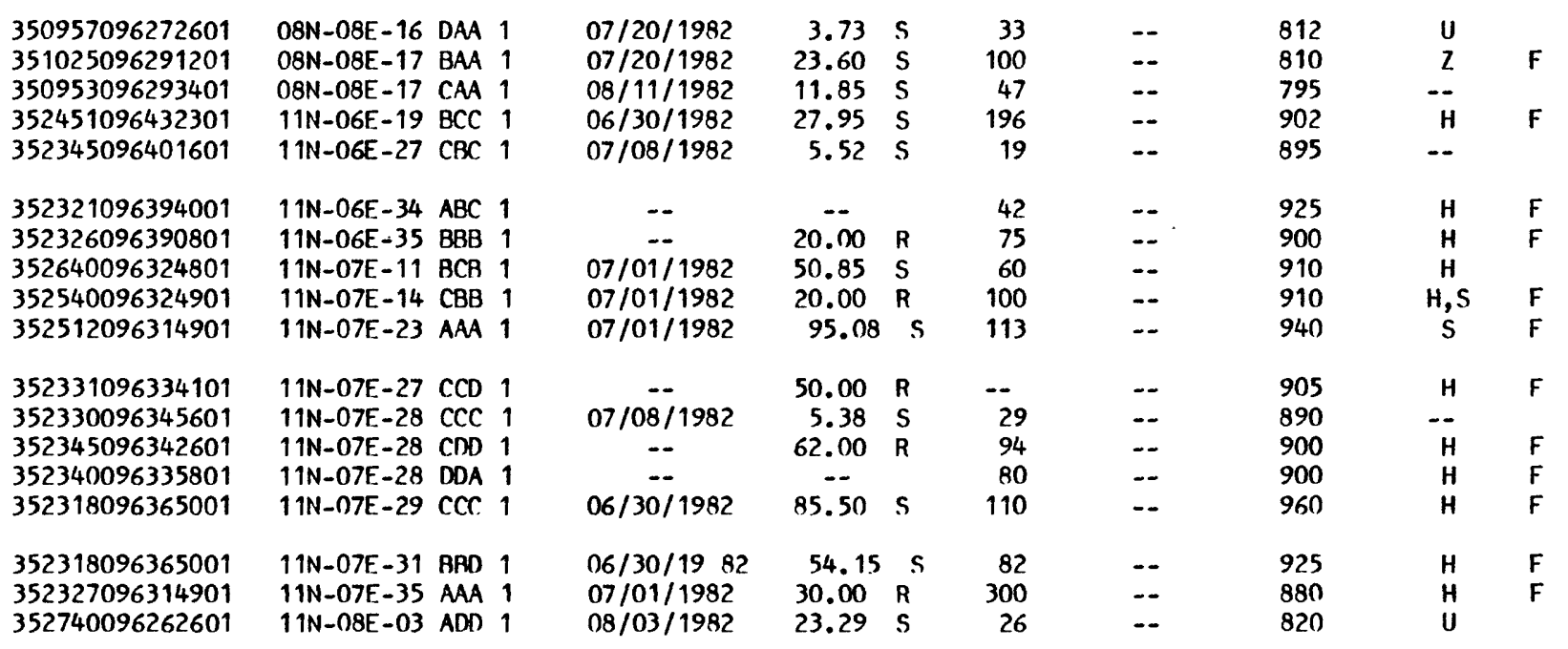


TABLE 2.--Field water-quality data for wells and test holes.

\begin{tabular}{|c|c|c|c|c|c|}
\hline & & $\begin{array}{c}\text { DATE } \\
\text { OUALITY }\end{array}$ & & $\begin{array}{c}\text { SPECIFIC. } \\
\text { CONDUCTANCE }\end{array}$ & \\
\hline SITE-ID & $\begin{array}{l}\text { LOCAL } \\
\text { NUMBER }\end{array}$ & $\begin{array}{c}\text { PARAMETERS } \\
\text { MEASURED }\end{array}$ & $\begin{array}{l}\text { TEMPERATURE } \\
\text { (DEGREES C) }\end{array}$ & $\begin{array}{l}(\mu \mathrm{MHOS} / \mathrm{CM} \\
\left.\text { AT } 25^{\circ} \mathrm{C}\right)\end{array}$ & $\begin{array}{c}\mathrm{pH} \\
\text { (UNITS) }\end{array}$ \\
\hline
\end{tabular}

\section{CANADIAN COUNTY}

\begin{tabular}{|c|c|}
\hline 352702097413301 & $11 N-05 W-02$ \\
\hline 352844097410601 & $12 N-05 W-25$ \\
\hline 352923097432501 & $12 N-05 W-27$ \\
\hline 352902097422701 & $12 N-05 W-27$ \\
\hline 352840097401901 & $12 N-05 W-36$ \\
\hline 35281509740190 & $12 N-05 W-36$ \\
\hline
\end{tabular}

$05 / 25 / 1982$

$05 / 27 / 1982$

$05 / 28 / 1982$

$05 / 27 / 1982$

$05 / 27 / 1982$

$05 / 27 / 1982$
18.0

16.5

18.8

19.0

19.0

19.0
731

1052

1210

1585

1160

1141
7.3

7.4

7.1

6.9

7.3

7.2

HUGHES COUNTY

350846096234401
350839096242301
350839096250501
351138096180201
350937096220801

351022096223301

350909096232201

351604096132401

351538096123401

351357096132101

351350096120901

351304096142201

351224096124601

351538096080301

351450096104801

351407096111601

351304096104501
$08 \mathrm{~N}-08 \mathrm{E}-23 \mathrm{CCD} 1$

$08 \mathrm{~N}-08 \mathrm{E}-25$ AAA 1

08N-08E-25 BAR 1

08N-09E-01 DAA 1

08N-09F-16 CCC 1

08N-09E-17 ABA 1

08N-09E-19 ADD 1

09N-10E-11 CAB 1

09N-10E-13 BBB 1

09N-10E-24 DDD 1

09N-10E-25 BAA 1

$09 N-10 E-27$ CDD 1

09N-10E-35 DAD 1

09N-11E-15 BAB 1

09N-11E-18-DDC 1

09N-11E-19 CDB 1

09N-11E-30 DDC 1
$07 / 20 / 1982$

$07 / 20 / 1982$

$07 / 20 / 1982$

$07 / 20 / 1982$

$07 / 20 / 1982$

$07 / 20 / 1982$

$07 / 20 / 1982$

$07 / 13 / 1982$

$07 / 13 / 1982$

$07 / 21 / 1982$

$07 / 21 / 1982$

$07 / 20 / 1982$

$07 / 21 / 1982$

$07 / 14 / 1982$

$07 / 21 / 1982$

$07 / 21 / 1982$

$07 / 21 / 1982$
22.0

19.0

22.0

21.0

20.0

18.0

21.0

27.5

19.0

19.0

19.0

16.5

24.0

18.0

19.0

20.0

21.0
1505

150

461

856

449

570

850

565

--

640

475

370

672

1357

1328

1560

1465
8.4

5.8

5.8

7.4

5.7

6.4

7.7

6.9

6.9

6.8

6.4

6.4

5.4

7.0

7.2

6.6

6.2

LINCOLN COUNTY

352847097080701

352752097080401

352823097072401
$12 \mathrm{~N}-02 \mathrm{E}-30-\mathrm{CDC}-01$

$12 \mathrm{~N}-02 \mathrm{E}-31$ CDC 1

$12 \mathrm{~N}-02 \mathrm{E}-32$ BCR 1
$06 / 15 / 1982$

$06 / 15 / 1982$

$06 / 15 / 1982$
22.0

17.5

20.0
683

250

795
6.8

5.6

6.8 
TABLE 2.--Field water-quality data for wells and test holes--Continued.

\begin{tabular}{|c|c|c|c|c|}
\hline & & $\begin{array}{c}\text { DATE } \\
\text { OUALITY }\end{array}$ & & $\begin{array}{c}\text { SPECIFIC } \\
\text { CONDUCTANCE }\end{array}$ \\
\hline SITE-ID & $\begin{array}{l}\text { LOCAL } \\
\text { NUMBER }\end{array}$ & $\begin{array}{c}\text { PARAMETERS } \\
\text { MEASURED }\end{array}$ & $\begin{array}{l}\text { TEMPERATURE } \\
\text { (DEGREES C) }\end{array}$ & $\begin{array}{l}(\mu \mathrm{MHOS} / \mathrm{CM} \\
\left.\text { AT } 25^{\circ} \mathrm{C}\right)\end{array}$ \\
\hline
\end{tabular}

MCINTOSH COUNTY

351958095541101

352234096474301

352410095482301

352418096470801
10N-13E-23 BAA 1 10N-14E-02 ABB 1 $11 \mathrm{~N}-14 \mathrm{E}-27$ AAD 1 $11 \mathrm{~N}-14 \mathrm{E}-36$ BBB 1
$07 / 14 / 1982$

$07 / 15 / 1982$

$07 / 15 / 1982$

$07 / 15 / 1982$
28.0

18.5

18.0

19.0
474

638

1121

575
6.0

6.7

7.5

7.3

OKFUSKEE COUNTY

\begin{abstract}
352012096180101
351930096180101

351944096165701

351959096112501

351926096080401

351928096065101

351923096052801

352020096032701

351935096021801

351853096003401

352728096324701

352619096243701

352432096231901
\end{abstract}

10N-09E-13 DDA 1 10N-09E-24 DAA 1 10N-10E-19 ADA 1 10N-11E-19 BBA 1 10N-11E-22 CAC 1

10N-11E-23 CAD 1 10N-11E-24 DAC 1 10N-12E-17 DBA 1 10N-12E-21 ADC 1 10N-12E-26 BDA 1

11N-07E-02 BCC 1 $11 N-08 E-12$ DCA 1 11N-09E-19 DDA 1
$07 / 12 / 1982$

$07 / 08 / 1982$

$07 / 08 / 1982$

$07 / 12 / 1982$

$07 / 13 / 1982$

$07 / 14 / 1982$

$07 / 14 / 1982$

$07 / 13 / 1982$

$07 / 14 / 1982$

$07 / 14 / 1982$

$07 / 06 / 1982$

$07 / 06 / 1982$

$07 / 07 / 1982$
22.0

17.0

18.0

17.5

17.0

20.0

16.0

22.0

21.0

22.0

19.0

17.0

18.0
862

688

967

696

2090

1430

445

1060

906

183

421

1058

412
6.7

7.6

5.9

6.9

6.8

7.1

7.0

6.5

6.6

5.7

6.4

7.2

8.0

OKLAHOMA COUNTY

\begin{tabular}{|c|c|}
\hline $\begin{array}{l}352748097271401 \\
352740097275301 \\
352724097280701 \\
352703097335301 \\
352651097315201\end{array}$ & $\begin{array}{l}11 N-02 W-06 \\
11 N-03 W-01 \\
11 N-03 W-01 \\
11 N-03 W-06 \\
11 N-03 W-08\end{array}$ \\
\hline $\begin{array}{l}352721097370601 \\
352749097375901 \\
352746097394601 \\
352721097391501 \\
352634097380801\end{array}$ & $\begin{array}{l}11 N-04 W-03 \\
11 N-04 W-04 \\
11 N-04 W-06 \\
11 N-04 W-06 \\
11 N-04 W-09\end{array}$ \\
\hline $\begin{array}{l}352624097345401 \\
352605097370201 \\
352702097381601 \\
353302097145101 \\
353209097140701\end{array}$ & $\begin{array}{l}11 N-04 W-12 \\
11 N-04 W-15 \\
11 N-04 W-05 \\
12 N-01 E-01 \\
12 N-01 E-07\end{array}$ \\
\hline
\end{tabular}

$06 / 04 / 1982$

$06 / 04 / 1982$

$06 / 03 / 1982$

$06 / 01 / 1982$

06/01/1982

$06 / 01 / 1982$

$06 / 01 / 1982$

$05 / 27 / 1982$

$05 / 27 / 1982$

$05 / 25 / 1982$

$06 / 01 / 1982$

$06 / 01 / 1982$

$05 / 28 / 1982$

$06 / 09 / 1982$

$06 / 10 / 1982$
17.0

17.0

19.5

18.0

19.0

21.0

18.0

17.0

23.0

20.4

19.0

17.0

19.0

20.0

16.9
477

422

573

534

482

1137

773

757

368

604

1507

3460

1719

720

805
6.8

7.0

7.8

7.9

6.8

7.3

7.0

7.8

7.2

8.3

6.9

6.7

7.1

7.0

6.9 
TABLE 2.--Field water-quality data for wells and test holes--Continued.

\begin{tabular}{|c|c|c|c|c|}
\hline & & $\begin{array}{c}\text { DATE } \\
\text { OUALITY }\end{array}$ & & $\begin{array}{c}\text { SPECIFIC } \\
\text { CONDUCTANCE }\end{array}$ \\
\hline SITE-ID & $\begin{array}{l}\text { LOCAL } \\
\text { NUMBER }\end{array}$ & $\begin{array}{l}\text { PARAMETERS } \\
\text { MEASURED }\end{array}$ & $\begin{array}{l}\text { TEMPERATURE } \\
\text { (DEGREES C) }\end{array}$ & $\begin{array}{l}(\mu \mathrm{MHOS} / \mathrm{CM} \\
\left.\text { AT } 25^{\circ} \mathrm{C}\right)\end{array}$ \\
\hline
\end{tabular}

$\mathrm{pH}$

(UNITS)

OKLAHOMA COUNTY--CONTINUED

\begin{tabular}{|c|c|c|c|c|c|c|c|}
\hline $\begin{array}{l}353142097144801 \\
353143097134901 \\
353116097115401 \\
353107097114201 \\
353117097125301\end{array}$ & $\begin{array}{l}12 \mathrm{~N}-01 \mathrm{E}-07 \\
12 \mathrm{~N}-01 \mathrm{E}-07 \\
12 \mathrm{~N}-01 \mathrm{E}-16 \\
12 \mathrm{~N}-01 \mathrm{E}-16 \\
12 \mathrm{~N}-01 \mathrm{E}-17\end{array}$ & $\begin{array}{l}\text { CBB } \\
\text { DAA } \\
\text { AAB } \\
\text { DDA } \\
\text { AAR }\end{array}$ & $\begin{array}{l}1 \\
1 \\
1 \\
1 \\
1\end{array}$ & $\begin{array}{l}06 / 09 / 1982 \\
06 / 14 / 1982 \\
06 / 14 / 1982 \\
06 / 14 / 1982 \\
06 / 14 / 1982\end{array}$ & $\begin{array}{l}17.0 \\
19.0 \\
20.0 \\
19.0 \\
19.0\end{array}$ & $\begin{array}{l}690 \\
951 \\
401 \\
691 \\
308\end{array}$ & $\begin{array}{l}6.8 \\
6.8 \\
7.1 \\
6.4 \\
6.2\end{array}$ \\
\hline $\begin{array}{l}353358097134401 \\
352940097152701 \\
352947097134601 \\
352949097124801 \\
353023097114201\end{array}$ & $\begin{array}{l}12 \mathrm{~N}-01 \mathrm{E}-17 \\
12 \mathrm{~N}-01 \mathrm{E}-19 \\
12 \mathrm{~N}-01 \mathrm{E}-20 \\
12 \mathrm{~N}-01 \mathrm{E}-20 \\
12 \mathrm{~N}-01 \mathrm{E}-21\end{array}$ & $\begin{array}{l}\text { BCB } \\
\text { BBA } \\
\text { CCB } \\
\text { DDA } \\
\text { AAA }\end{array}$ & $\begin{array}{l}1 \\
1 \\
1 \\
1 \\
1\end{array}$ & $\begin{array}{c}-- \\
06 / 10 / 1982 \\
06 / 14 / 1982 \\
06 / 14 / 1982 \\
06 / 14 / 1982\end{array}$ & $\begin{array}{l}-- \\
19.0 \\
18.5 \\
22.0 \\
19.0\end{array}$ & $\begin{array}{l}- \\
400 \\
308 \\
433 \\
704\end{array}$ & $\begin{array}{l}6.9 \\
6.5 \\
6.3 \\
6.4 \\
7.2\end{array}$ \\
\hline $\begin{array}{l}352951097115701 \\
352941097114101 \\
352938097103801 \\
352948097093601 \\
352945097092201\end{array}$ & $\begin{array}{l}12 N-01 E-21 \\
12 N-01 E-21 \\
12 N-01 E-22 \\
12 N-01 E-23 \\
12 N-01 E-24\end{array}$ & $\begin{array}{l}\text { DAD } \\
\text { DDD } \\
\text { DDD } \\
\text { DAD } \\
\text { CCA }\end{array}$ & $\begin{array}{l}1 \\
1 \\
1 \\
1 \\
1\end{array}$ & $\begin{array}{l}06 / 14 / 1982 \\
06 / 14 / 1982 \\
06 / 14 / 1982 \\
06 / 14 / 1982 \\
06 / 14 / 1982\end{array}$ & $\begin{array}{l}19.0 \\
20.0 \\
18.0 \\
18.5 \\
17.5\end{array}$ & $\begin{array}{r}388 \\
463 \\
884 \\
1671 \\
1756\end{array}$ & $\begin{array}{l}6.7 \\
6.3 \\
6.6 \\
7.1 \\
6.9\end{array}$ \\
\hline $\begin{array}{l}352930097084701 \\
352927097092901 \\
352808097093001 \\
353214097150601 \\
353727097230701\end{array}$ & $\begin{array}{l}12 N-01 E-25 \\
12 N-01 E-25 \\
12 N-01 E-36 \\
12 N-01 W-01 \\
12 N-02 W 11\end{array}$ & $\begin{array}{l}\text { ABA } \\
\text { BBC } \\
\text { CBC } \\
\text { DCD } \\
\text { BCA }\end{array}$ & $\begin{array}{l}1 \\
1 \\
1 \\
1 \\
1\end{array}$ & $\begin{array}{l}06 / 15 / 1982 \\
06 / 14 / 1982 \\
06 / 15 / 1982 \\
06 / 09 / 1982 \\
06 / 07 / 1982\end{array}$ & $\begin{array}{l}17.0 \\
19.0 \\
17.0 \\
77.0 \\
19.0\end{array}$ & $\begin{array}{r}613 \\
970 \\
359 \\
616 \\
1033\end{array}$ & $\begin{array}{l}6.6 \\
6.7 \\
6.1 \\
6.8 \\
7.0\end{array}$ \\
\hline $\begin{array}{l}353303097224701 \\
353231097221801 \\
353214097224401 \\
353157097232101 \\
353210097240001\end{array}$ & $\begin{array}{l}12 N-02 W-02 \\
12 N-02 W-02 \\
12 N-02 W-02 \\
12 N-02 W-10 \\
12 N-02 W-10\end{array}$ & $\begin{array}{l}\text { ABB } \\
\text { DAA } \\
\text { DCC } \\
\text { ADA } \\
\text { BAB }\end{array}$ & $\begin{array}{l}1 \\
1 \\
1 \\
1 \\
1\end{array}$ & $\begin{array}{l}06 / 07 / 1982 \\
06 / 08 / 1982 \\
06 / 07 / 1982 \\
06 / 07 / 1982 \\
06 / 07 / 1982\end{array}$ & $\begin{array}{l}19.0 \\
21.0 \\
19.0 \\
17.0 \\
20.0\end{array}$ & $\begin{array}{l}398 \\
575 \\
420 \\
388 \\
610\end{array}$ & $\begin{array}{l}7.2 \\
6.8 \\
7.3 \\
7.1 \\
6.8\end{array}$ \\
\hline $\begin{array}{l}353138097231601 \\
353126097224001 \\
353154097221101 \\
353054097232001 \\
353054097235901\end{array}$ & $\begin{array}{l}12 N-02 W-11 \\
12 N-02 W-11 \\
12 N-02 W-12 \\
12 N-02 W-15 \\
12 N-02 W-15\end{array}$ & $\begin{array}{l}\text { CBC } \\
\text { DCC } \\
\text { BCB } \\
\text { ADD } \\
\text { BDC }\end{array}$ & $\begin{array}{l}1 \\
1 \\
1 \\
1 \\
1\end{array}$ & $\begin{array}{l}06 / 07 / 1982 \\
06 / 08 / 1982 \\
06 / 08 / 1982 \\
06 / 07 / 1982 \\
06 / 07 / 1982\end{array}$ & $\begin{array}{l}18.0 \\
17.0 \\
20.5 \\
20.0 \\
19.0\end{array}$ & $\begin{array}{r}664 \\
594 \\
473 \\
575 \\
1010\end{array}$ & $\begin{array}{l}7.1 \\
6.4 \\
6.7 \\
6.6 \\
6.8\end{array}$ \\
\hline $\begin{array}{l}353147097251601 \\
353102097252901 \\
353024097261601 \\
352947097262701 \\
352939097254901\end{array}$ & $\begin{array}{l}12 N-02 W-16 \\
12 N-02 W-17 \\
12 N-02 W-20 \\
12 N-02 W-20 \\
12 N-02 W-20\end{array}$ & $\begin{array}{l}\text { BBB } \\
\text { DDD } \\
\text { BBA } \\
\text { CCB } \\
\text { DCD }\end{array}$ & $\begin{array}{l}1 \\
1 \\
1 \\
1 \\
1\end{array}$ & $\begin{array}{l}06 / 04 / 1982 \\
06 / 04 / 1982 \\
06 / 04 / 1982 \\
06 / 04 / 1982 \\
06 / 04 / 1982\end{array}$ & $\begin{array}{r}17.5 \\
18.0 \\
19.0 \\
19.0 \\
7.0\end{array}$ & $\begin{array}{l}518 \\
510 \\
763 \\
691 \\
836\end{array}$ & $\begin{array}{l}7.9 \\
7.0 \\
6.9 \\
7.5 \\
6.7\end{array}$ \\
\hline
\end{tabular}


TABLE 2.--Field water-quality data for wells and test holes--Continued.

\begin{tabular}{|c|c|c|c|c|}
\hline & & $\begin{array}{c}\text { DATE } \\
\text { QUALITY }\end{array}$ & & $\begin{array}{c}\text { SPECIFIC } \\
\text { CONDUCTANCE }\end{array}$ \\
\hline SITE-ID & $\begin{array}{l}\text { LOCAL } \\
\text { NUMBER }\end{array}$ & $\begin{array}{l}\text { PARAMETERS } \\
\text { MEASURED }\end{array}$ & $\begin{array}{l}\text { TEMPERATURE } \\
\text { (DEGREES C) }\end{array}$ & $\begin{array}{l}(\mu \mathrm{MHOS} / \mathrm{CM} \\
\left.\text { AT } 25^{\circ} \mathrm{C}\right)\end{array}$ \\
\hline
\end{tabular}

OKLAHOMA COUNTY--CONTINUED

\begin{tabular}{|c|c|c|c|c|c|c|c|}
\hline $\begin{array}{l}353017097245201 \\
353019097242101 \\
352855097252301 \\
352933097271701 \\
352934097272401\end{array}$ & $\begin{array}{l}12 N-02 W-21 \\
12 N-02 W-22 \\
12 N-02 W-28 \\
12 N-02 W-30 \\
12 N-02 W-30\end{array}$ & $\begin{array}{l}\text { ABC } \\
\text { RBC } \\
\text { CCR } \\
\text { BAB } \\
\text { BBA }\end{array}$ & $\begin{array}{l}1 \\
1 \\
1 \\
1 \\
1\end{array}$ & $\begin{array}{l}06 / 09 / 1982 \\
06 / 08 / 1982 \\
06 / 04 / 1982 \\
06 / 04 / 1982 \\
06 / 03 / 1982\end{array}$ & $\begin{array}{l}18.0 \\
23.0 \\
17.0 \\
17.0 \\
19.0\end{array}$ & $\begin{array}{r}781 \\
880 \\
935 \\
524 \\
1206\end{array}$ & $\begin{array}{l}6.8 \\
6.6 \\
7.3 \\
7.5 \\
7.0\end{array}$ \\
\hline $\begin{array}{l}352840097263201 \\
352838097260101 \\
353253097391601 \\
353145097394701 \\
352853097393101\end{array}$ & $\begin{array}{l}12 N-02 W-31 \\
12 N-02 W-32 \\
12 N-04 W-06 \\
12 N-04 W-07 \\
12 N-04 W-30\end{array}$ & $\begin{array}{l}\text { AAA } \\
\text { BAD } \\
\text { AAD } \\
\text { CAA } \\
\text { CAA }\end{array}$ & $\begin{array}{l}1 \\
1 \\
1 \\
1 \\
1\end{array}$ & $\begin{array}{l}06 / 03 / 1982 \\
06 / 03 / 1982 \\
05 / 25 / 1982 \\
05 / 25 / 1982 \\
05 / 27 / 1982\end{array}$ & $\begin{array}{l}18.5 \\
19.2 \\
18.0 \\
18.0 \\
18.0\end{array}$ & $\begin{array}{r}1050 \\
583 \\
622 \\
10169 \\
579\end{array}$ & $\begin{array}{l}7.0 \\
7.2 \\
7.3 \\
7.4 \\
6.5\end{array}$ \\
\hline $\begin{array}{l}343000097394401 \\
352816097390601 \\
352753097380501 \\
352821097360101 \\
353328097144001\end{array}$ & $\begin{array}{l}12 N-04 W-30 \\
12 N-04 W-32 \\
12 N-04 W-33 \\
12 N-04 W-35 \\
13 N-01 E-31\end{array}$ & $\begin{array}{l}\text { DBC } \\
\text { CBA } \\
\text { CCD } \\
\text { BBC } \\
\text { CBA }\end{array}$ & $\begin{array}{l}1 \\
1 \\
1 \\
1 \\
1\end{array}$ & $\begin{array}{l}05 / 27 / 1982 \\
05 / 28 / 1982 \\
05 / 29 / 1982 \\
06 / 01 / 1982 \\
06 / 09 / 1982\end{array}$ & $\begin{array}{l}17.0 \\
20.0 \\
17.5 \\
21.0 \\
17.8\end{array}$ & $\begin{array}{r}795 \\
806 \\
598 \\
1007 \\
736\end{array}$ & $\begin{array}{l}7.1 \\
6.7 \\
7.2 \\
7.0 \\
7.1\end{array}$ \\
\hline $\begin{array}{l}353606097175701 \\
353622097194501 \\
353618097201401 \\
353539097203801 \\
353515097201101\end{array}$ & $\begin{array}{l}13 N-01 W-15 \\
13 N-01 W-17 \\
13 N-01 W-18 \\
13 N-01 W-19 \\
13 N-01 W-19\end{array}$ & $\begin{array}{l}\text { CBB } \\
\text { BAC } \\
\text { ADA } \\
\text { ABB } \\
\text { ADD }\end{array}$ & $\begin{array}{l}1 \\
1 \\
1 \\
1 \\
1\end{array}$ & $\begin{array}{l}06 / 08 / 1982 \\
06 / 08 / 1982 \\
06 / 08 / 1982 \\
06 / 08 / 1982 \\
06 / 07 / 1982\end{array}$ & $\begin{array}{l}19.0 \\
19.0 \\
19.0 \\
19.0 \\
23.0\end{array}$ & $\begin{array}{l}642 \\
688 \\
560 \\
872 \\
405\end{array}$ & $\begin{array}{l}7.0 \\
7.1 \\
6.9 \\
7.0 \\
6.5\end{array}$ \\
\hline $\begin{array}{l}353452097203701 \\
353451097183101 \\
353532097170201 \\
353528097175901 \\
353450097172701\end{array}$ & $\begin{array}{l}13 N-01 W-19 \\
13 N-01 W-21 \\
13 N-01 W-22 \\
13 N-01 W-22 \\
13 N-01 W-22\end{array}$ & $\begin{array}{l}D C C \\
D C C \\
A D D \\
B B C \\
D C C\end{array}$ & $\begin{array}{l}1 \\
1 \\
1 \\
1 \\
1\end{array}$ & $\begin{array}{l}06 / 09 / 1982 \\
05 / 20 / 1982 \\
06 / 09 / 1982 \\
06 / 09 / 1982 \\
06 / 09 / 1982\end{array}$ & $\begin{array}{l}19.0 \\
18.0 \\
22.0 \\
18.5 \\
19.0\end{array}$ & $\begin{array}{r}476 \\
386 \\
477 \\
1060 \\
780\end{array}$ & $\begin{array}{l}6.9 \\
6.8 \\
6.8 \\
6.8 \\
6.9\end{array}$ \\
\hline $\begin{array}{l}353457097162701 \\
353429097154401 \\
353445097155601 \\
353356097162301 \\
353403097210901\end{array}$ & $\begin{array}{l}13 N-01 W-23 \\
13 N-01 W-25 \\
13 N-01 W-26 \\
13 N-01 W-26 \\
13 N-01 W-30\end{array}$ & $\begin{array}{l}\text { CDA } \\
\text { BCA } \\
\text { AAA } \\
\text { DCC } \\
\text { CCB }\end{array}$ & $\begin{array}{l}1 \\
1 \\
1 \\
1 \\
1\end{array}$ & $\begin{array}{l}05 / 20 / 1982 \\
06 / 09 / 1982 \\
06 / 09 / 1982 \\
06 / 10 / 1982 \\
06 / 07 / 1982\end{array}$ & $\begin{array}{l}18.0 \\
22.0 \\
21.0 \\
20.5 \\
18.0\end{array}$ & $\begin{array}{r}500 \\
535 \\
483 \\
1635 \\
204\end{array}$ & $\begin{array}{l}7.2 \\
6.5 \\
6.8 \\
6.9 \\
5.9\end{array}$ \\
\hline $\begin{array}{l}353313097162601 \\
353305097155301 \\
353513097222201 \\
353530097211701 \\
353454097213901\end{array}$ & $\begin{array}{l}13 N-01 W-35 \\
13 N-01 W-35 \\
13 N-02 W-23 \\
13 N-02 W-24 \\
13 N-02 W-24\end{array}$ & $\begin{array}{l}\text { CDA } \\
\text { DDD } \\
\text { DAA } \\
\text { AAD } \\
\text { DCC }\end{array}$ & $\begin{array}{l}1 \\
1 \\
1 \\
1 \\
1\end{array}$ & $\begin{array}{l}06 / 10 / 1982 \\
06 / 10 / 1982 \\
06 / 09 / 1982 \\
06 / 08 / 1982 \\
06 / 08 / 1982\end{array}$ & $\begin{array}{l}22.0 \\
18.0 \\
18.0 \\
19.0 \\
18.5\end{array}$ & $\begin{array}{r}529 \\
1622 \\
6239 \\
768 \\
490\end{array}$ & $\begin{array}{l}6.8 \\
6.6 \\
7.0 \\
7.0 \\
6.9\end{array}$ \\
\hline
\end{tabular}


TABLE 2.--Field water-quality data for wells and test holes--Continued.

\begin{tabular}{|c|c|c|c|c|}
\hline & & $\begin{array}{c}\text { DATE } \\
\text { DUALITY }\end{array}$ & & $\begin{array}{c}\text { SPECIFIC } \\
\text { CONDUCTANCE }\end{array}$ \\
\hline SITE-ID & $\begin{array}{l}\text { LOCAL } \\
\text { NUMBER }\end{array}$ & $\begin{array}{c}\text { PARAMETERS } \\
\text { MEASURED }\end{array}$ & $\begin{array}{l}\text { TEMPERATURE } \\
\text { (DEGREES C) }\end{array}$ & $\begin{array}{l}(\mu \mathrm{MHOS} / \mathrm{CM} \\
\left.\text { AT } 25^{\circ} \mathrm{C}\right)\end{array}$ \\
\hline
\end{tabular}

$\mathrm{pH}$

(UNITS)

OKLAHOMA COUNTY--CONTINUED

$\begin{array}{llll}353447097215801 & 13 N-02 W-25 & \text { BAB } & 1 \\ 353351097230701 & 13 N-02 W-35 & \text { BBD } & 1 \\ 353306097231801 & 13 N-02 W-35 & \text { CCC } & 1 \\ 353354097213901 & 13 N-02 W-36 & \text { ABB } & 1 \\ 353205097215301 & 13 N-02 W-36 & \text { CDC } & 1\end{array}$

$06 / 08 / 1982$

$06 / 07 / 1982$

$06 / 07 / 1982$

$06 / 07 / 1982$

$06 / 08 / 1982$
17.0

19.0

18.0

20.0

18.0

20.0

$07 / 15 / 1982$

352307095531301

$11 \mathrm{~N}-13 \mathrm{E}-36 \mathrm{BDC} 1$

POTTAWATOMIE COUNTY

352227097010301
352155097000401
352219097020901
352145097010601
352139097000301
352141096585901
352141096595201
352059097000001
352056096585901
352140096581601

352006096574201
352046097575601
352000096594001
351909096580001
351956096565801
351915096572601
351923096565301
351907096561601
351819096561501
351817096573001
352152096493001
352220096513001
352110096502901
352139096502301
352001096501901

10N-03E-05 BBC 1

10N-03E-05 DAA 1

10N-03E-06 BCB 1

10N-03E-06 DDD 1

10N-03E-08 AAD 1

10N-03E-09 AAA 1

10N-03E-09 BBA 1

10N-03E-09 CCB 1

10N-03E-09 DDD 1

10N-03E-10 ABA 1

10N-03E-14 CCA 1

10N-03E-15 AAA 1

10N-03E-16 CDC 1

10N-03E-22 DDD 1

10N-03E-23 AAA 1

10N-03E-23 CDA 1

10N-03E-23 DAD 1

$10 \mathrm{~N}-03 \mathrm{E}-24$ DCC 1

10N-03E-25 CAC 1

10N-03E-26 CDD 1

10N-04E-01 DDR 1

10N-04F-02 RCB 1

10N-04E-11 DAA 1

10N-04F-12 BRB 1

$10 \mathrm{~N}-04 \mathrm{E}-13$ CCD 1
$06 / 18 / 1982$

$06 / 18 / 1982$

$06 / 17 / 1982$

$06 / 17 / 1982$

$06 / 18 / 1982$

$06 / 21 / 1982$

$06 / 21 / 1982$

$06 / 18 / 1982$

$06 / 18 / 1982$

$06 / 22 / 1982$

$06 / 21 / 1982$

$06 / 22 / 1982$

$06 / 18 / 1982$

$06 / 22 / 1982$

$06 / 22 / 1982$

$06 / 21 / 1982$

$06 / 22 / 1982$

$06 / 22 / 1982$

$06 / 22 / 1982$

$06 / 22 / 1982$

$06 / 23 / 1982$

$06 / 23 / 1982$

$06 / 24 / 1982$

$06 / 24 / 1982$

$06 / 24 / 1982$
19.0

19.0

19.5

19.5

18.0

19.0

17.0

19.0

17.0

19.0

19.0

19.0

19.0

19.0

18.0

21.0

18.0

17.0

21.0

21.0

21.0

19.0

22.0

20.5

22.0
364

1226

846

232

367

7.2

7.0

7.0

6.0

6.8

352

6.0

430

2530

944

1208

2420

274

952

963

745

361

6.8

7.8

8.4

8.2

7.6

5.7

6.6

6.6

7.1

6.3

1529

6.5

418

543

827

6.2

5.8

6.7

532

6.7

736

6.6

572

1291

6.2

6.7

955

7.1

1754

6.8

929

6.7

466

6.7

1353

6.7

452

6.5

1018 
TABLE 2.--Field water-quality data for wells and test holes--Continued.

\begin{tabular}{|c|c|c|c|c|}
\hline & & $\begin{array}{c}\text { DATE } \\
\text { DIJALITY }\end{array}$ & & $\begin{array}{c}\text { SPECIFIC } \\
\text { CONDUCTANCE }\end{array}$ \\
\hline SITE-ID & $\begin{array}{l}\text { LOCAL } \\
\text { NUMBER }\end{array}$ & $\begin{array}{c}\text { PARAMETERS } \\
\text { MEASURED }\end{array}$ & $\begin{array}{l}\text { TEMPERATURE } \\
\text { (DEGREES C) }\end{array}$ & $\begin{array}{l}(\mu \mathrm{MHOS} / \mathrm{CM} \\
\left.\text { AT } 25^{\circ} \mathrm{C}\right)\end{array}$ \\
\hline
\end{tabular}

POTTAWATOMIE COUNTY--CONTINUED

\begin{tabular}{|c|c|c|c|c|c|c|c|}
\hline $\begin{array}{l}352000096511901 \\
352026096523101 \\
351959096523901 \\
351910096553001 \\
351920096540601\end{array}$ & $\begin{array}{l}10 N-04 E-14 \\
10 N-04 E-15 \\
10 N-04 E-16 \\
10 N-04 E-19 \\
10 N-04 E-20\end{array}$ & $\begin{array}{l}C C D \\
B B C \\
D D D \\
C D C \\
D B C\end{array}$ & $\begin{array}{l}1 \\
1 \\
1 \\
1 \\
1\end{array}$ & $\begin{array}{l}06 / 23 / 1982 \\
06 / 23 / 1982 \\
06 / 23 / 1982 \\
06 / 22 / 1982 \\
06 / 23 / 1982\end{array}$ & $\begin{array}{l}22.0 \\
18.5 \\
18.0 \\
18.0 \\
16.5\end{array}$ & $\begin{array}{r}719 \\
1007 \\
858 \\
417 \\
818\end{array}$ & $\begin{array}{l}6.3 \\
6.8 \\
7.2 \\
8.3 \\
6.9\end{array}$ \\
\hline $\begin{array}{l}351904096523401 \\
351905096533901 \\
351818096532701 \\
351818096541201 \\
351836096552801\end{array}$ & $\begin{array}{l}10 N-04 E-27 \\
10 N-04 E-28 \\
10 N-04 E-28 \\
10 N-04 E-29 \\
10 N-04 E-30\end{array}$ & $\begin{array}{l}\text { BBA } \\
\text { BBR } \\
\text { CCD } \\
\text { DCC } \\
\text { CAB }\end{array}$ & $\begin{array}{l}1 \\
1 \\
1 \\
1 \\
1\end{array}$ & $\begin{array}{l}06 / 23 / 1982 \\
06 / 23 / 1982 \\
06 / 23 / 1982 \\
06 / 23 / 1982 \\
06 / 22 / 1982\end{array}$ & $\begin{array}{l}16.0 \\
18.0 \\
18.0 \\
20.0 \\
18.0\end{array}$ & $\begin{array}{l}529 \\
572 \\
650 \\
579 \\
640\end{array}$ & $\begin{array}{l}7.2 \\
7.6 \\
7.1 \\
6.7 \\
6.9\end{array}$ \\
\hline $\begin{array}{l}351753096545001 \\
352130096492401 \\
352104096492601 \\
352511097033101 \\
352702097055401\end{array}$ & $\begin{array}{l}10 N-04 E-31 \\
10 N-05 E-07 \\
10 N-05 E-07 \\
11 N-01 E-23 \\
11 N-02 E-04\end{array}$ & $\begin{array}{l}\text { ADD } \\
B C B \\
C C B \\
\text { ARA } \\
\text { CDD }\end{array}$ & $\begin{array}{l}1 \\
1 \\
1 \\
1 \\
1\end{array}$ & $\begin{array}{l}06 / 24 / 1982 \\
06 / 24 / 1982 \\
06 / 23 / 1982 \\
06 / 16 / 1982 \\
06 / 16 / 1982\end{array}$ & $\begin{array}{l}18.0 \\
17.0 \\
22.0 \\
20.0 \\
20.0\end{array}$ & $\begin{array}{l}539 \\
770 \\
767 \\
682 \\
456\end{array}$ & $\begin{array}{l}7.2 \\
6.5 \\
6.5 \\
7.6 \\
7.3\end{array}$ \\
\hline $\begin{array}{l}352718097071301 \\
352658097264501 \\
352647097062201 \\
352630097061501 \\
352656097042801\end{array}$ & $\begin{array}{l}11 \mathrm{~N}-02 \mathrm{E}-05 \\
11 \mathrm{~N}-02 \mathrm{E}-05 \\
11 \mathrm{~N}-02 \mathrm{E}-09 \\
11 \mathrm{~N}-02 \mathrm{E}-09 \\
11 \mathrm{~N}-02 \mathrm{E}-10\end{array}$ & $\begin{array}{l}\text { CBA } \\
\text { DCD } \\
\text { BBC } \\
\text { CBA } \\
\text { AAB }\end{array}$ & $\begin{array}{l}1 \\
1 \\
1 \\
1 \\
1\end{array}$ & $\begin{array}{l}06 / 15 / 1982 \\
06 / 15 / 1982 \\
06 / 16 / 1982 \\
06 / 16 / 1982 \\
06 / 16 / 1982\end{array}$ & $\begin{array}{l}18.5 \\
18.0 \\
18.0 \\
18.0 \\
17.0\end{array}$ & $\begin{array}{l}412 \\
407 \\
338 \\
380 \\
837\end{array}$ & $\begin{array}{l}5.9 \\
5.9 \\
6.0 \\
6.1 \\
8.4\end{array}$ \\
\hline $\begin{array}{l}352642097034801 \\
352619097023001 \\
352619097032001 \\
352540097021601 \\
352540097043301\end{array}$ & $\begin{array}{l}11 \mathrm{~N}-02 \mathrm{E}-11 \\
11 \mathrm{~N}-02 \mathrm{E}-11 \\
11 \mathrm{~N}-02 \mathrm{E}-12 \\
11 \mathrm{~N}-02 \mathrm{E}-13 \\
11 \mathrm{~N}-02 \mathrm{E}-15\end{array}$ & $\begin{array}{l}\text { BDA } \\
\text { DAD } \\
\text { CDD } \\
A D D \\
A D C\end{array}$ & $\begin{array}{l}1 \\
1 \\
1 \\
1 \\
1\end{array}$ & $\begin{array}{l}06 / 16 / 1982 \\
06 / 16 / 1982 \\
06 / 18 / 1982 \\
06 / 16 / 1982 \\
06 / 16 / 1982\end{array}$ & $\begin{array}{l}20.5 \\
22.0 \\
18.0 \\
17.0 \\
18.0\end{array}$ & $\begin{array}{l}571 \\
602 \\
761 \\
694 \\
316\end{array}$ & $\begin{array}{l}8.2 \\
6.5 \\
8.0 \\
8.4 \\
5.7\end{array}$ \\
\hline $\begin{array}{l}352450097031301 \\
352330097023901 \\
352407097031801 \\
350238097230401 \\
352233097010301\end{array}$ & $\begin{array}{l}11 N-02 E-24 \\
11 N-02 E-25 \\
11 N-02 E-26 \\
11 N-02 E-36 \\
11 N-03 E-05\end{array}$ & $\begin{array}{l}\text { BCC } \\
\text { DCC } \\
\text { ADA } \\
\text { ACC } \\
\text { BBB }\end{array}$ & $\begin{array}{l}1 \\
1 \\
1 \\
1 \\
1\end{array}$ & $\begin{array}{l}06 / 16 / 1982 \\
06 / 17 / 1982 \\
06 / 16 / 1982 \\
06 / 17 / 1982 \\
06 / 17 / 1982\end{array}$ & $\begin{array}{l}18.5 \\
18.0 \\
21.0 \\
19.0 \\
20.0\end{array}$ & $\begin{array}{l}718 \\
554 \\
751 \\
673 \\
578\end{array}$ & $\begin{array}{l}7.3 \\
8.4 \\
7.2 \\
8.0 \\
6.7\end{array}$ \\
\hline $\begin{array}{l}352459097020601 \\
352330097021001 \\
352419097015301 \\
352330097014601 \\
352313097011901\end{array}$ & $\begin{array}{l}11 N-03 E-19 \\
11 N-03 E-25 \\
11 N-03 E-30 \\
11 N-03 E-30 \\
11 N-03 E-31\end{array}$ & $\begin{array}{l}\text { BCC } \\
\text { DDD } \\
\text { BBA } \\
\text { CDC } \\
\text { ADB }\end{array}$ & $\begin{array}{l}1 \\
1 \\
1 \\
1 \\
1\end{array}$ & $\begin{array}{l}06 / 16 / 1982 \\
06 / 17 / 1982 \\
06 / 17 / 1982 \\
06 / 17 / 1982 \\
06 / 11 / 1982\end{array}$ & $\begin{array}{l}18.0 \\
19.0 \\
20.0 \\
17.0 \\
17.0\end{array}$ & $\begin{array}{l}577 \\
572 \\
361 \\
450 \\
251\end{array}$ & $\begin{array}{l}6.6 \\
7.6 \\
7.7 \\
8.2 \\
6.7\end{array}$ \\
\hline
\end{tabular}


TABLE 2.--Field water-quality data for wells and test holes--Continued.

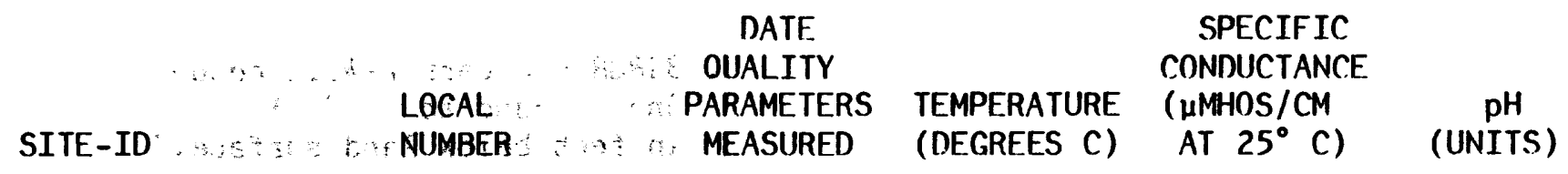

POTTAWATOMIE COUNTY--CONTINUED

\begin{tabular}{|c|c|c|c|c|c|c|c|}
\hline $\begin{array}{l}352302097010601 \\
352513096502701 \\
352603096494001 \\
352600096205501 \\
352600096513201\end{array}$ & $\begin{array}{l}11 N-03 E-31 \\
11 N-04 E-13 \\
11 N-04 E-14 \\
11 N-04 E-14 \\
11 N-04 E-15\end{array}$ & $\begin{array}{l}A D D \\
C C C \\
A A B \\
A B B \\
A A A\end{array}$ & $\begin{array}{r}1 \\
1 \\
-1 \\
1 \\
1\end{array}$ & $\begin{array}{r}06 / 17 / 1982 \\
06 / 24 / 1982 \\
-06 / 24 / 1982 \\
06 / 24 / 1982 \\
06 / 24 / 1982\end{array}$ & $\begin{array}{l}20.0 \\
18.0 \\
19.0 \\
17.0 \\
18.0\end{array}$ & $\begin{array}{l}204 \\
290 \\
789 \\
682 \\
736\end{array}$ & $\begin{array}{l}6.2 \\
5.8 \\
6.7 \\
7.6 \\
7.0\end{array}$ \\
\hline $\begin{array}{l}352507096514101 \\
352429096523401 \\
352414096520201 \\
352327096522001 \\
352552096434601\end{array}$ & $\begin{array}{l}11 \mathrm{~N}-04 \mathrm{E}-22 \\
11 \mathrm{~N}-04 \mathrm{E}-22 \\
11 \mathrm{~N}-04 \mathrm{E}-27 \\
11 \mathrm{~N}-04 \mathrm{E}-34 \\
11 \mathrm{~N}-05 \mathrm{E}-13\end{array}$ & $\begin{array}{l}\text { AAA } \\
\text { CCB } \\
\text { ABB } \\
\text { BAB } \\
\text { BAC }\end{array}$ & $\begin{array}{l}1 \\
1 \\
1 \\
1 \\
1\end{array}$ & $\begin{array}{r}06 / 24 / 1982 \\
06 / 28 / 1982 \\
06 / 24 / 1982 \\
06 / 24 / 1982 \\
06 / 29 / 1982\end{array}$ & $\begin{array}{l}18.0 \\
18.5 \\
19.0 \\
17.0 \\
19.0\end{array}$ & $\begin{array}{l}425 \\
742 \\
382 \\
578 \\
742\end{array}$ & $\begin{array}{l}6.2 \\
6.6 \\
6.6 \\
6.9 \\
6.8\end{array}$ \\
\hline $\begin{array}{l}352525096444301 \\
352428096485601 \\
352425096480901 \\
352509096463701 \\
352453096453601\end{array}$ & $\begin{array}{l}11 \mathrm{~N}-05 \mathrm{E}-14 \\
11 \mathrm{~N}-05 \mathrm{E}-19 \\
11 \mathrm{~N}-05 \mathrm{E}-20 \\
11 \mathrm{~N}-05 \mathrm{E}-21 \\
11 \mathrm{~N}-05 \mathrm{E}-22\end{array}$ & $\begin{array}{l}\text { CAD } \\
\text { CDA } \\
\text { CED } \\
\text { ABA } \\
\text { ACA }\end{array}$ & $\begin{array}{l}1 \\
1 \\
1 \\
1 \\
1\end{array}$ & $\begin{array}{l}06 / 29 / 1982 \\
06 / 25 / 1982 \\
06 / 25 / 1982 \\
06 / 28 / 1982 \\
06 / 29 / 1982\end{array}$ & $\begin{array}{l}19.0 \\
21.0 \\
18.0 \\
19.0 \\
19.5\end{array}$ & $\begin{array}{l}335 \\
515 \\
992 \\
455 \\
818\end{array}$ & $\begin{array}{l}5.8 \\
6.2 \\
6.6 \\
5.7 \\
7.8\end{array}$ \\
\hline $\begin{array}{l}352507096451101 \\
352535096415301 \\
352353096391401 \\
352720096334901 \\
352437096360001\end{array}$ & $\begin{array}{l}11 \mathrm{~N}-05 \mathrm{E}-23 \\
11 \mathrm{~N}-06 \mathrm{E}-17 \\
11 \mathrm{~N}-06 \mathrm{E}-27 \\
11 \mathrm{~N}-07 \mathrm{E}-03 \\
11 \mathrm{~N}-07 \mathrm{E}-20\end{array}$ & $\begin{array}{l}\text { BBB } \\
\text { CBA } \\
\text { ACD } \\
\text { CBR } \\
\text { CBC }\end{array}$ & $\begin{array}{l}1 \\
1 \\
1 \\
1 \\
1\end{array}$ & $\begin{array}{l}06 / 29 / 1982 \\
06 / 29 / 1982 \\
06 / 29 / 1982 \\
07 / 02 / 1982 \\
07 / 02 / 1982\end{array}$ & $\begin{array}{l}20.0 \\
19.0 \\
19.0 \\
17.5 \\
17.0\end{array}$ & $\begin{array}{l}472 \\
811 \\
420 \\
584 \\
363\end{array}$ & $\begin{array}{l}7.3 \\
6.6 \\
7.1 \\
6.8 \\
6.6\end{array}$ \\
\hline 352447096345901 & $11 N-07 E-20$ & DAA & 1 & $07 / 02 / 1982$ & 19.0 & 603 & 6.6 \\
\hline
\end{tabular}

SEMINOLE COUNTY

$\begin{array}{llll}351025096291201 & 08 \mathrm{~N}-08 \mathrm{E}-17 & \mathrm{BAA} & 1 \\ 352451096432301 & 11 \mathrm{~N}-06 \mathrm{E}-19 & \mathrm{BCC} & 1 \\ 352321096394001 & 11 \mathrm{~N}-06 \mathrm{E}-34 & \mathrm{ABC} & 1 \\ 352326096390801 & 11 \mathrm{~N}-06 \mathrm{E}-35 & \mathrm{BBB} & 1 \\ 352540096324901 & 11 \mathrm{~N}-07 \mathrm{E}-14 & \mathrm{CBB} & 1 \\ & & & \\ 352512096314901 & 11 \mathrm{~N}-07 \mathrm{E}-23 & \mathrm{AAA} & 1 \\ 352331096334101 & 11 \mathrm{~N}-07 \mathrm{E}-27 & \mathrm{CCD} & 1 \\ 352345096342601 & 11 \mathrm{~N}-07 \mathrm{E}-28 & \mathrm{CDD} & 1 \\ 352340096335801 & 11 \mathrm{~N}-07 \mathrm{E}-28 & \mathrm{DDA} & 1 \\ 352327096314901 & 11 \mathrm{~N}-07 \mathrm{E}-35 & \mathrm{AAA} & 1\end{array}$

$07 / 20 / 1982$

$06 / 30 / 1982$

$06 / 30 / 1982$

$06 / 30 / 1982$

$07 / 01 / 1982$

$07 / 01 / 1982$

$07 / 01 / 1982$

$06 / 30 / 1982$

$07 / 01 / 1982$

$07 / 01 / 1982$
19.0

19.0

17.0

19.0

19.0

18.5

18.0

18.0

19.0

18.0
630

1118

1015

768

317

556

257

518

511

650
8.0

7.5

7.1

7.0

5.7

5.7

6.1

6.8

6.7

6.6 
TABLE 3.--Lithologic logs of test holes.

[Abbreviations used: 110ALVM = Alluvium, 318GRBR $=$ Garber - Wellington Formations, 322VMOS = Vamoosa Formation; Snds = Sandstone, Sncl = Sandy clay, Slsn = Siltstone; depths are in feet below land surface.]

Test Hole no. 1

Site ID: 352700097404501

Local number: 11N-05W-01 DCC 1

Date drilled: 06/28/82

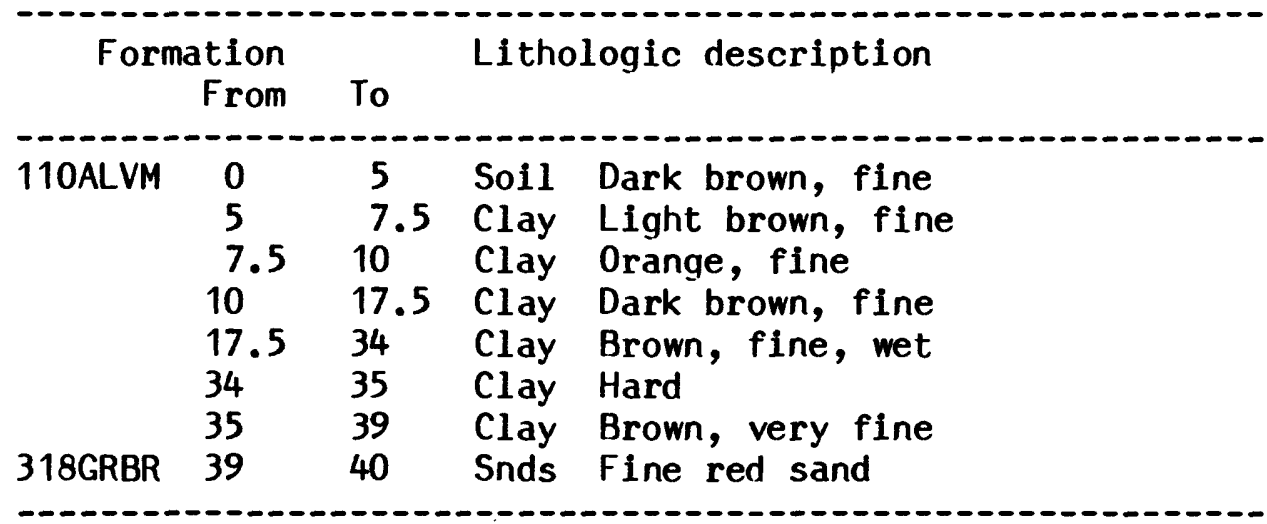

Test Hole no. 2

Site ID: 352700097384101

Local number: $11 \mathrm{~N}-04 \mathrm{~W}-05$ CDD 1

Date drilled: 06/29/82

$\begin{array}{rrrr}\text { 110ALVM } & 0 & 2.5 & \text { Soil Brown, some clay } \\ & 2.5 & 9.6 & \text { Clay Brown, very fine } \\ & 9.6 & 28.5 & \text { Sand Medium } \\ 318 G R B R & 33.5 & 33.5 & \text { Sand Coarse, dark } \\ & 35 & \text { Snds Red, very fine }\end{array}$

Test Hole no. 3

Site ID: 352741097324901

Local number: $11 \mathrm{~N}-03 \mathrm{~W}-05$ BBC 1

Date drilled: 06/29/82

$\begin{array}{lllll}\text { 110ALVM } & 0 & 2.5 & \text { Soil Brown, fine } \\ & 2.5 & 10 & \text { Sncl } & \text { Dark brown, fine } \\ 10 & 12.5 & \text { Sncl Tan, very fine } \\ 12.5 & 17 & \text { Sand Tan, very fine } \\ 17 & 34 & \text { Sncl Fine, saturated } \\ & 34 & 38 & \text { Sand Fine, grayish tan } \\ 318 \text { GRBR } & 38 & 39 & \text { Slsn Red }\end{array}$


TABLE 3.--Lithologic logs of test holes.--Continued.

Test Hole no. 4

Site ID: 352715097314901

Local number: 11N-03W-05 DAD 1

Date drilled: 06/29/82

\begin{tabular}{|c|c|c|c|c|}
\hline Form & $\begin{array}{l}\text { ation } \\
\text { From }\end{array}$ & To & Lithe & logic description \\
\hline 110ALVM & $\begin{array}{l}0 \\
2.5 \\
13 \\
17.5 \\
31\end{array}$ & $\begin{array}{l}2.5 \\
13 \\
17.5 \\
31 \\
32.5\end{array}$ & $\begin{array}{l}\text { Sdst } \\
\text { Sand } \\
\text { Clay } \\
\text { Sand } \\
\text { Snds }\end{array}$ & $\begin{array}{l}\text { Medium } \\
\text { Fine to medium } \\
\text { Brown, fine } \\
\text { Medium to coarse } \\
\text { Red, fine }\end{array}$ \\
\hline
\end{tabular}

Test Hole no. 5

Site ID: 352806097411901

Local number: $12 \mathrm{~N}-05 \mathrm{~W}-36 \mathrm{CBC} 1$

Date drilled: $06 / 30 / 82$

$\begin{array}{lllll}\text { 110ALVM } & 0 & 2.5 & \text { Soil } & \text { Very fine } \\ & 2.5 & 10 & \text { Clay } & \text { Red, fine } \\ & 10 & 20 & \text { Clay } & \text { Brown, very fine } \\ & 20 & 38 & \text { Sand } & \text { Brown, very fine } \\ 318 \text { GRBR } & 38 & 40 & \text { Snds Red, very fine }\end{array}$

Test Hole no. 6

Site ID: 352726097325601

Local number: 11N-03W-06 ADD 1

Date drilled: 06/30/82

\begin{tabular}{lrrrl} 
110ALVM & \multicolumn{1}{c}{} & 2.5 & Soil Fine \\
& 2.5 & 7.5 & Sand Fine \\
& 7.5 & 17.5 & Sncl Fine, brown \\
& 17.5 & 38.5 & Sand Fine to coarse \\
318 GRBR & 38.5 & 39 & Snds Red, very fine
\end{tabular}

Test Hole no. 7

Site ID: 352726097294301

Local number: $11 \mathrm{~N}-03 \mathrm{~W}-03$ ADD 1

Date drilled: 06/30/82

\begin{tabular}{lllll}
\hline $110 A L V M$ & 0 & 15 & Sand & Brown, fine \\
& 15 & 22.5 & Sncl \\
& 22.5 & 44 & Sand & Fint brown, fine to medium \\
318 GRBR & 44 & 45 & Snds Red, very fine
\end{tabular}


TABLE 3.--Lithologic logs of test holes.--Continued.

Test Hole no. 8

Site ID: 352743097293701

Local number: $11 \mathrm{~N}-03 \mathrm{~W}-02$ BBC 1

Date drilled: $06 / 30 / 82$

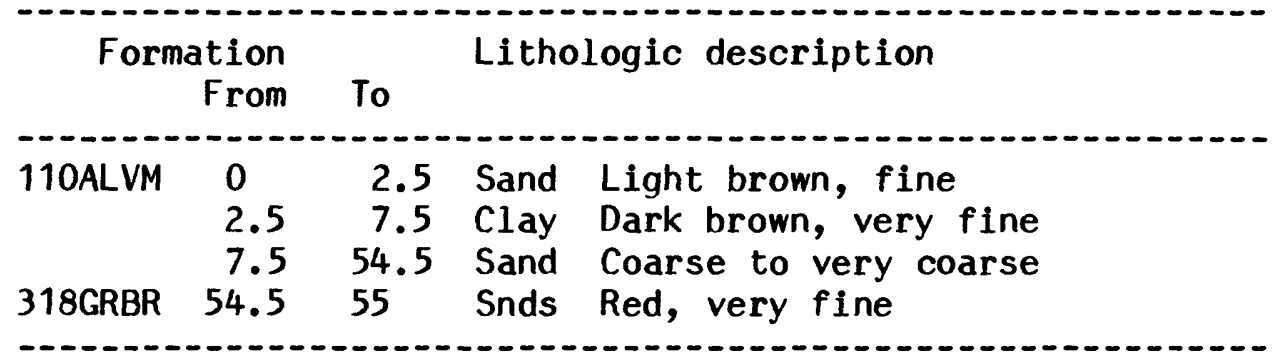

Test Hole no. 9

Site ID: 353644097231901

Local number: $12 \mathrm{~N}-02 \mathrm{~W}-15$ AAA 1

Date drilled: 07/01/82

$\begin{array}{lllll}\text { 110ALVM } & 0 & 2.5 & \text { Soil Red-brown, fine } \\ & 2.5 & 7.5 & \text { Sand Dark brown, fine } \\ & 7.5 & 12.5 & \text { Sand Brown, very fine } \\ 12.5 & 20 & \text { Sand Light brown, fine } \\ & 20 & 30 & \text { Sand Light brown, fine } \\ \text { 318GRBR } & 38 & 38 & \text { Sand Light brown, fine } \\ & 40 & \text { Snds Red, medium, wet }\end{array}$

Test Hole no. 10

Site ID: 353544097204601

Local number: 13N-01W-18 CDD 1

Date drilled: 07/01/82

$\begin{array}{llll}\text { 110ALVM } & 0 & 2.5 & \text { Soil Brown, some clay } \\ & 2.5 & 20 & \text { Clay Brown, very fine } \\ 20 & 32.5 \text { Sand Light brown, fine } \\ 32.5 & 42.5 \text { Sand Medium } \\ 42.5 & 43.5 \text { Snds Red, very fine } \\ 318 \text { GRBR } & 43.5 & 47.5 & \text { Snds Light red, wet }\end{array}$


TABLE 3.--Lithologic logs of test holes.--Continued.

Test Hole no. 11

Site ID: 354001097135501

Local number: $12 \mathrm{~N}-01 \mathrm{E}-18$ ADB 1

Date drilled: $07 / 06 / 82$

\begin{tabular}{llll}
$\begin{array}{c}\text { Formation } \\
\text { From }\end{array}$ & \multicolumn{1}{c}{ To } \\
110ALVM & 0 & 5 & Sand Brown, fine \\
& 5 & 17.5 & Sand Brown, medium \\
& 17.5 & 20 & Clay Dark brown, very fine \\
20 & 49 & Sand Medium to very coarse \\
318GRBR & 49 & 50 & Snds Red, very fine
\end{tabular}

Test Hole no. 12

Site ID: 352957097124201

Local number: $12 \mathrm{~N}-01 \mathrm{E}-21$ CBB 1

Date drilled: 07/06/82

\begin{tabular}{|c|c|c|c|c|}
\hline 110ALVM & $\begin{array}{l}0 \\
5 \\
7.5 \\
15 \\
17.5 \\
20 \\
25 \\
32.5 \\
37.5 \\
40\end{array}$ & $\begin{array}{l}5 \\
7.5 \\
15 \\
17.5 \\
20 \\
25 \\
32.5 \\
37.5 \\
40 \\
41\end{array}$ & $\begin{array}{l}\text { Soil } \\
\text { Clay } \\
\text { Sand } \\
\text { Sand } \\
\text { Sand } \\
\text { Sand } \\
\text { Sand } \\
\text { Sand } \\
\text { Sand } \\
\text { Snds }\end{array}$ & $\begin{array}{l}\text { Brown, fine } \\
\text { Dark brown, very fine } \\
\text { Light brown, fine } \\
\text { Darker, fine } \\
\text { Darker, fine } \\
\text { Darker, medium } \\
\text { Brown, coarse } \\
\text { Brown, medium } \\
\text { Brown, coarse } \\
\text { Red, fine sand }\end{array}$ \\
\hline
\end{tabular}

Test hole no. 13

Site ID: 352748097055201

Local number: 11N-02E-04 BAA 1

Date drilled: 07/06/82

$\begin{array}{lllll} & & & \\ & 2.5 & 10 & \text { Clay B Brown } \\ & 10 & 22.5 & \text { Clay Light brown, fine } \\ & 22.5 & 45 & \text { Sand Light brown, fine } \\ & 45 & 47 & \text { Snds Red, coarse } \\ \text { 318GRBR } & 47 & 47.5 & \text { Snds Red, saturated }\end{array}$


TABLE 3.--Lithologic logs of test holes.--Continued.

Test Hole no. 14

Site ID: 352208097010601

Local number: 10N-03E-06 DAA 1

Date drilled: 07/07/82

\begin{tabular}{l}
$\begin{array}{c}\text { Formation } \\
\text { From }\end{array}$ \\
\hline To
\end{tabular}

Test Hole no. 15

Site ID: 351908096572101

Local number: 10N-03E-23 DCC 1

Date drilled: 07/07/82

$110 \mathrm{ALVM}$
$318 \mathrm{GRBR} 34$ $35 \quad \begin{aligned} & \text { Sand Fine to coarse } \\ & \text { Snds Red, very fine }\end{aligned}$

Test Hole no. 16

Site ID: 352512096490701

Local number: $11 \mathrm{~N}-04 \mathrm{E}-24$ ABB 1

Date drilled: 07/07/82

$\begin{array}{lllll}\text { 110ALVM } & 0 & 2.5 & \text { Soil Brown, some clay } \\ & 2.5 & 15 & \text { Sand Light brown, fine } \\ & 15 & 32.5 & \text { Sand Brown, medium } \\ 318 G R B R & 32.5 & 35 & \text { Snds Red, very fine }\end{array}$

Test Hole no. 17

Site ID: 352420096463601

Local number: $11 \mathrm{~N}-05 \mathrm{E}-21$ DCD 1

Date drilled: 07/07/82

$\begin{array}{ccccl}\text { 110ALVM } & 0 & 2.5 & \text { Clay } & \text { Dark brown, fine } \\ & 2.5 & 5 & \text { Clay Brown, fine, wet } \\ 5 & 10 & \text { Sand Light brown, fine } \\ 10 & 20 & \text { Sand Brown, medium } \\ 20 & 32.5 & \text { Sand Brown, coarse } \\ 32.5 & 35 & \text { Clay Blue-green, fine }\end{array}$


TABLE 3.--Lithologic logs of test holes.--Continued.

Test Hole no. 18

Site ID: 352510096485901

Local number: 11N-05E-19 BAA 1

Date drilled: 07/07/82

\begin{tabular}{c}
$\begin{array}{c}\text { Formation } \\
\text { From }\end{array}$ \\
\hline 110 To
\end{tabular}

Test Hole no. 19

Site ID: 352330096345601

Local number: 11N-07E-28 CCC 1

Date drilled: 07/08/82

$\begin{array}{cccc}110 A L V M & 0 & 2.5 & \text { Soil Brown, some clay } \\ & 2.5 & 7.5 & \text { Clay Bawn, fine } \\ 7.5 & 10 & \text { Sand Brown, fine } \\ 10 & 20 & \text { Clay Brown, fine } \\ 20 & 30 & \text { Sand } \\ 318 \text { GRBR } & 30 & 33 & \text { Snds Red, very fine }\end{array}$

Test Hole no. 20

Site ID: 352345096401601

Local number: 11N-06E-27 CBC 1

Date drilled: 07/08/82

\begin{tabular}{lllll}
\hline 110ALVM & 0 & 2.5 & Sand & Brown, medium \\
& 2.5 & 12.5 & Clay Fine to very fine \\
& 12.5 & 19 & Sand & Fine to medium \\
318GRBR & 19 & 20 & Snds Red, medium
\end{tabular}

Test Hole no. 21

Site ID: 352444096405901

Local number: 11N-06E-20 ADD 1

Date drilled: 07/08/82

$\begin{array}{lccc}\text { 110ALVM } & 0 & 32.5 & \text { Sand } \\ \text { 322VMOS } & 32.5 & 33 & \text { Snds } \\ \text { Bluish-gray, fine }\end{array}$


TABLE 3.--Lithologic logs of test holes:-Côntinuềd.

Test Hole no. 22

Site ID: 352509096420601

Local number: 11N-06E-19 AAA 1

Date drilled: $07 / 08 / 82$

$\begin{gathered}\text { Formation } \\ \text { From }\end{gathered}$
To

Test Hole no. 23

Site ID: 352422096341201

Local number: 11N-07E-28 ABA 1

Date drilled: 07/08/82

$\begin{array}{lllll}\text { 110ALVM } & 0 & 2.5 & \text { Soil } & \text { Brown, some sand } \\ & 2.5 & 12.5 & \text { Sand } & \text { Brown, very fine } \\ 12.5 & 15 & \text { Clay Brown, very fine } \\ 15 & 27.5 & \text { Sand } \\ 27.5 & 32.5 & \text { Sand Brown, very fine } \\ 32.5 & 35 & \text { Sand Brown, fine } \\ 35 & 36 & \text { Clay Red, fine }\end{array}$

Test Hole no. 24

Site ID: 352752096344701

Local number: 11N-07E-33 CDD 1

Date drilled: $08 / 02 / 82$

$\begin{array}{llll}\text { 110ALVM } & 0 & 45 & \text { Clay Brown, fine } \\ \text { 322VMOS } & 45 & 47.5 \text { Snds Bluish, very fine }\end{array}$

Test Hole no. 25

Site ID: 352614096302101

Local number: $11 \mathrm{~N}-08 \mathrm{E}=07^{-} \mathrm{DDA}^{-} 1$

Date drilled: 08/02/82

$\begin{array}{lrrr}\text { 110ALVM } & 0 & 15 & \text { Clay Brown, very fine } \\ & 15 & 39 & \text { Sand Medium to fine } \\ \text { 322VMOS } & 39 & 40 & \text { Snds Bluish, medium }\end{array}$


TABLE 3.--Lithologic logs of test holes.--Continued.

Test Hole no. 26

Site ID: 352808096262701

Local number: 12N-08E-35 $\mathrm{CBC} 1$

Date drilled: 08/03/82

\begin{tabular}{|c|c|c|c|c|}
\hline Form & $\begin{array}{l}\text { ation } \\
\text { From }\end{array}$ & To & Lith & logic description \\
\hline 110ALVM & $\begin{array}{l}0 \\
7.5 \\
20 \\
25 \\
27.5 \\
40 \\
42\end{array}$ & $\begin{array}{l}7.5 \\
20 \\
25 \\
27.5 \\
40 \\
42 \\
42.5\end{array}$ & $\begin{array}{l}\text { Clay } \\
\text { Clay } \\
\text { Clay } \\
\text { Clay } \\
\text { Sand } \\
\text { Sand } \\
\text { Sand }\end{array}$ & $\begin{array}{l}\text { Dark brown, fine } \\
\text { Brown, very fine } \\
\text { Light brown, fine } \\
\text { Light brown, fine } \\
\text { Light brown, fine } \\
\text { Light brown, medium } \\
\text { Blue-gray, fine }\end{array}$ \\
\hline
\end{tabular}

Test Hole no. 27

Site ID: 352659096252301

Local number: $11 \mathrm{~N}-08 \mathrm{E}-12$ BBB 1

Date drilled: 08/03/82

$\begin{array}{lrrr}110 A L V M & 0 & 5 & \text { Clay } \\ & 5 & 36 & \text { Sand Very fine } \\ \text { 322VMOS } & 36 & 37.5 & \text { Snds Bluish, very fine }\end{array}$

Test Hole no. 28

Site ID: 352545096244301

Local number: $11 \mathrm{~N}-08 \mathrm{E}-13$ ACD 1

Date drilled: 08/03/82

$\begin{array}{llll}2.5 & 12.5 & \text { Clay } & \text { Brown, very fine } \\ 12.5 & 13 & \text { Sand } & \text { Brown, very fine } \\ 13 & 13.2 & \text { Sand Yellow, very fine }\end{array}$

Test Hole no. 29

Site ID: 352740096262601

Local number: 11N-08E-03 ADD 1

Date drilled: 08/03/82

$\begin{array}{lllll}110 A L V M & 0 & 2.5 & \text { Soil } & \text { Brown, some clay } \\ & 2.5 & 17.5 & \text { Clay } & \text { Brown, very fine } \\ & 17.5 & 35 & \text { Sand } & \text { Brown, very fine } \\ & 35 & 40 & \text { Sand } & \text { Brown, medium } \\ \text { 322VMOS } & 40 & 41 & \text { Snds Gray, fine }\end{array}$


TABLE 3.--Lithologic logs of test holes.--Continued.

Test Hole no. 30

Site ID: 352238096235001

Local number: 10N-09E-06 BAA 1

Date drilled: 08/04/82

Formation
From

Test Hole no. 31

Site ID: 352213096210801

Local number: 10N-09E-15 BBB 1

Date drilled: 08/03/82

$\begin{array}{lllll}\text { 110ALVM } & 0 & 5 & \text { Clay } & \text { Black, very fine } \\ & 5 & 7.5 & \text { Clay Dark brown, very fine } \\ & 7.5 & 12.5 & \text { Clay Yellow-brown, fine } \\ & 12.5 & 30 & \text { Sand Light brown, very fine } \\ & 30 & 37.5 & \text { Sand Light brown, medium } \\ 322 \text { VMOS } & 37.5 & 45 & \text { Sand Dark brown, medium } \\ \text { 35 } & 46 & \text { Clay Blue-gray, very fine }\end{array}$

Test Hole no. 32

Site ID: 352237096221301

Local number: 10N-09E-05 AAA 1

Date drilled: 08/04/82

$\begin{array}{lll}\text { 110ALVM } 0 & 28 & \text { Sand Fine to medium } \\ 322 \text { VMOS } 28 & 30 & \text { Snds Bluish gray, fine }\end{array}$

Test Hole no. 33

Site ID: 351959096185801

Local number: 10N-09E-24 BBB 1

Date drilled: 08/04/82

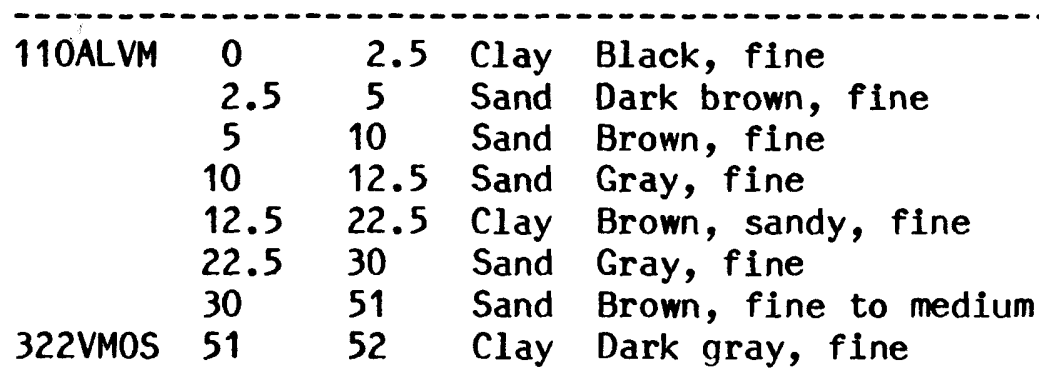


TABLE 3.--Lithologic logs of test holes.--Continued.

Test Hole no. 34

Site ID: 352007096175901

Local number: 10N-09E-13 DDD 1

Date drilled: 08/04/82

\begin{tabular}{cccc}
$\begin{array}{c}\text { Formation } \\
\text { From }\end{array}$ & To & Lithologic description \\
\hline 110ALVM & 0 & 2.5 & Soil Brown, some sand \\
& 2.5 & 5 & Sand Brown, very fine \\
& 5 & 10 & Clay Brown, very fine \\
10 & 22.5 & Sand Dark brown, medium \\
& 22.5 & 30 & Sand Dark brown, coarse \\
322 VMOS & 30 & 31.5 & Snds Gray, very fine
\end{tabular}

Test Hole no. 35

Site ID: 352054096144601

Local number: $10 \mathrm{~N}-10 \mathrm{E}-10$ CCC 1

Date drilled: 08/05/82

$\begin{array}{llll}\text { 11OALVM } & 0 & 2.5 & \text { Sand Light brown, very fine } \\ & 2.5 & 15 & \text { Clay Brown, very fine } \\ & 15 & 36 & \text { Sand Very fine to medium } \\ 322 \text { VMOS } & 36 & 37.5 & \text { Snds Bluish, very fine }\end{array}$

Test Hole no. 36

Site ID: 352000096110401

Local number: $10 \mathrm{~N}-11 \mathrm{E}-18$ DCC 1

Date drilled: 08/04/82

$\begin{array}{llll}\text { 110ALVM } & 0 & 5 & \text { Sand Dark brown, very fine } \\ & 5 & 12.5 & \text { Clay Brown, very fine } \\ & 12.5 & 42.5 & \text { Sand Brown, medium } \\ 322 \text { VMOS } & 42.5 & 43 & \text { Clay Gray, coarse }\end{array}$

Test Hole no. 37

Site ID: 351546096093001

Local number: 09N-11E-08 DDD 1

Date drilled: 08/05/82

$\begin{array}{lllll}\text { 110ALVM } & 0 & 2.5 & \text { Soil } & \text { Brown, some sand } \\ & 2.5 & 15 & \text { Clay } & \text { Brown, very fine } \\ & 15 & 22.5 & \text { Sand } \\ & 22.5 & 37.5 & \text { Clay } & \text { Brown, very fine } \\ 322 \text { VMOS } & 37.5 & 42.5 & \text { Sand } & \text { Beige, very fine } \\ \text { 32.5 } & 43 & \text { Snds } & \text { Gray, very fine }\end{array}$


TABLE 3.--Lithologic logs of test holes.--Continued.

Test Hole no. 38

Site ID: 351605096131301

Local number: 09N-10E-11 CAA 1

Date drilled: 08/05/82

\begin{tabular}{|c|c|c|c|c|}
\hline Forn & $\begin{array}{l}\text { ation } \\
\text { From }\end{array}$ & To & Litho & logic description \\
\hline $\begin{array}{l}\text { 110ALVM } \\
\text { 322VMOS }\end{array}$ & $\begin{array}{r}0 \\
2.5 \\
30.5\end{array}$ & $\begin{array}{r}2.5 \\
30.5 \\
32.5\end{array}$ & $\begin{array}{l}\text { Clay } \\
\text { Sand } \\
\text { Snds }\end{array}$ & $\begin{array}{l}\text { Brown, very fine } \\
\text { Very fine to fine } \\
\text { Gray, very fine }\end{array}$ \\
\hline
\end{tabular}

Test Hole no. 39

Site ID: 351538096113401

Local number: 09N-11E-18 BBB 1

Date drilled: 08/05/82

$\begin{array}{cccc}110 A L V M & 0 & 10 & \text { Sand Brown, very fine } \\ & 10 & 12.5 & \text { Sand Light brown, fine } \\ 12.5 & 17 & \text { Sand Dark brown, fine } \\ 17 & 32.5 & \text { Sand Dark brown, medium } \\ 32.5 & 33.8 & \text { Clay Blue-gray, very fine }\end{array}$

Test Hole no. 40

Site ID: 351915096073801

Local number: 10N-11E-22 DCA 1

Date drilled: 08/05/82

$\begin{array}{lllll}\text { 110ALVM } & 0 & 2.5 & \text { Sand Brown, very fine } \\ & 2.5 & 7.5 & \text { Clay Brown, very fine } \\ & 7.5 & 10 & \text { Sand Brown, very fine } \\ \text { 322VMOS } & 12.5 & 12.5 & \text { Clay Brown, very fine } \\ & 15 & \text { Clay Gray, very fine }\end{array}$

Test Hole no. 41

Site ID: 352016096061901

Local number: 10N-11E-14 DAD 1

Date drilled: $08 / 05 / 82$

$\begin{array}{lrll}\text { 110ALVM } & 0 & 15 & \text { Clay Brown, very fine } \\ & 15 & 39 & \text { Sand Very fine to medium } \\ 322 \text { VMOS } & 39 & 40 & \text { Snds Bluish-gray, fine }\end{array}$


TABLE 3.--Lithologic logs of test holes.--Continued.

Test Hole no. 42

Site ID: 351817096020301

Local number: 10N-12E-27 CCC 1

Date drilled: 08/05/82

\begin{tabular}{|c|c|c|c|c|c|c|}
\hline Forn & $\begin{array}{l}\text { ation } \\
\text { From }\end{array}$ & To & Litho & $\operatorname{logic} d t$ & escri & tion \\
\hline 110ALVM & $\begin{array}{l}0 \\
2.5 \\
15 \\
17.5\end{array}$ & $\begin{array}{l}2.5 \\
15 \\
17.5 \\
19\end{array}$ & $\begin{array}{l}\text { Soil } \\
\text { Clay } \\
\text { Sand } \\
\text { Snds }\end{array}$ & $\begin{array}{l}\text { Brown, } \\
\text { Brown, } \\
\text { Brown, } \\
\text { Black, }\end{array}$ & $\begin{array}{l}\text { some } \\
\text { very } \\
\text { very } \\
\text { fine }\end{array}$ & $\begin{array}{l}\text { clay } \\
\text { fine } \\
\text { fine }\end{array}$ \\
\hline
\end{tabular}

Test Hole no. 43

Site ID: 352303095530701

Local number: $11 \mathrm{~N}-13 \mathrm{E}-36$ BDD 1

Date drilled: 08/09/82

$\begin{array}{lllll}\text { 110ALVM } & 0 & 2.5 & \text { Sand Brown, very fine } \\ 2.5 & 7.5 & \text { Sand Light brown, very fine } \\ 7.5 & 17.5 & \text { Sand Brown, very fine } \\ 17.5 & 20 & \text { Sand Light brown, very fine } \\ 20 & 22.5 & \text { Sand } \\ 22.5 & 25 & \text { Sand } & \text { Gray-brown, very fine } \\ 25 & 37.5 & \text { Sand } & \text { Brown, fine } \\ 37.5 & 38 & \text { Clay Gray, very fine }\end{array}$

Test Hole no. 44

Site ID: 352420095472601

Local number: $11 \mathrm{~N}-14 \mathrm{E}-26$ ABB 1

Date drilled: $08 / 10 / 82$

$\begin{array}{cccc}110 A L V M & 0 & 2.5 & \text { Sncl Brown, very fine } \\ 2.5 & 5 & \text { Clay } & \text { Brown, very fine } \\ 5 & 12.5 & \text { Clay } \\ 12.5 & 17.5 & \text { Sand } & \text { Brown, very fine } \\ 17.5 & 32.5 & \text { Clay } & \text { Brown, very fine } \\ 32.5 & 35 & \text { Clay Hard }\end{array}$

Test Hole no. 45

Site ID: 351142096145301

Local number: $08 \mathrm{~N}-10 \mathrm{E}-04$ DAA 1

Date drilled: $08 / 10 / 82$

\begin{tabular}{|c|c|c|c|c|}
\hline $\begin{array}{l}\text { 110ALVM } \\
322 \mathrm{VMOS}\end{array}$ & $\begin{array}{r}0 \\
21\end{array}$ & $\begin{array}{l}21 \\
22.5\end{array}$ & $\begin{array}{l}\text { Clay } \\
\text { Snds }\end{array}$ & $\begin{array}{l}\text { Brown, very fine } \\
\text { Blue, very fine }\end{array}$ \\
\hline
\end{tabular}


TABLE 3.--Lithologic logs of test holes.--Continued.

Test Hole no. 46

Site ID: 351030096234801

Local number: 08N-09E-09 DDD 1

Date drilled: 08/11/82

\begin{tabular}{|c|c|c|c|c|}
\hline Forr & $\begin{array}{r}\text { ation } \\
\text { From }\end{array}$ & To & Litho & logic description \\
\hline 110ALVM & $\begin{array}{r}0 \\
2.5 \\
7.5 \\
17.5\end{array}$ & $\begin{array}{r}2.5 \\
7.5 \\
17.5 \\
18\end{array}$ & $\begin{array}{l}\text { Soll } \\
\text { Clay } \\
\text { Sand } \\
\text { Snds }\end{array}$ & $\begin{array}{l}\text { Brown, some clay } \\
\text { Brown, very fine } \\
\text { Brown, very fine } \\
\text { Gray, very fine }\end{array}$ \\
\hline
\end{tabular}

Test Hole no. 47

Site ID: 351213096200201

Local number: $09 \mathrm{~N}-09 \mathrm{E}-35$ CCC 1

Date drilled: $08 / 10 / 82$

$\begin{array}{lllll}110 A L V M & 0 & 2.5 & \text { Sand Yellow-brown, very fine } \\ & 2.5 & 7.5 & \text { Clay Black, very fine } \\ 7.5 & 12.5 & \text { Clay Yellow-brown, very fine } \\ 12.5 & 20 & \text { Clay Brown, very fine }\end{array}$

Test Hole no. 48

Site ID: 350945096240401

Local number: $08 \mathrm{~N}-09 \mathrm{E}-18 \mathrm{CDA} 1$

Date drilled: $08 / 12 / 82$

\begin{tabular}{lllll}
\hline $110 A L V M$ & 0 & 5 & Sand Light brown, fine \\
& 5 & 7.5 & Clay Red, fine \\
& 7.5 & 27.5 & Clay Brown, very fine \\
322 VMOS & 28.5 & 28 & Sand Brown, fine \\
30 & 30 & Clay Gray, fine
\end{tabular}

Test Hole no. 49

Site ID: 350953096293401

Local number: $08 \mathrm{~N}-08 \mathrm{E}-17$ CAA 1

Date drilled: $08 / 11 / 82$

$\begin{array}{lllll}\text { 110ALVM } & 0 & 2.5 & \text { Soil } & \text { Brown, sandy } \\ & 2.5 & 47.5 & \text { Clay } & \text { Brown, very fine } \\ \text { 322VMOS } & 47.5 & 49 & \text { Snds Gray, very fine }\end{array}$



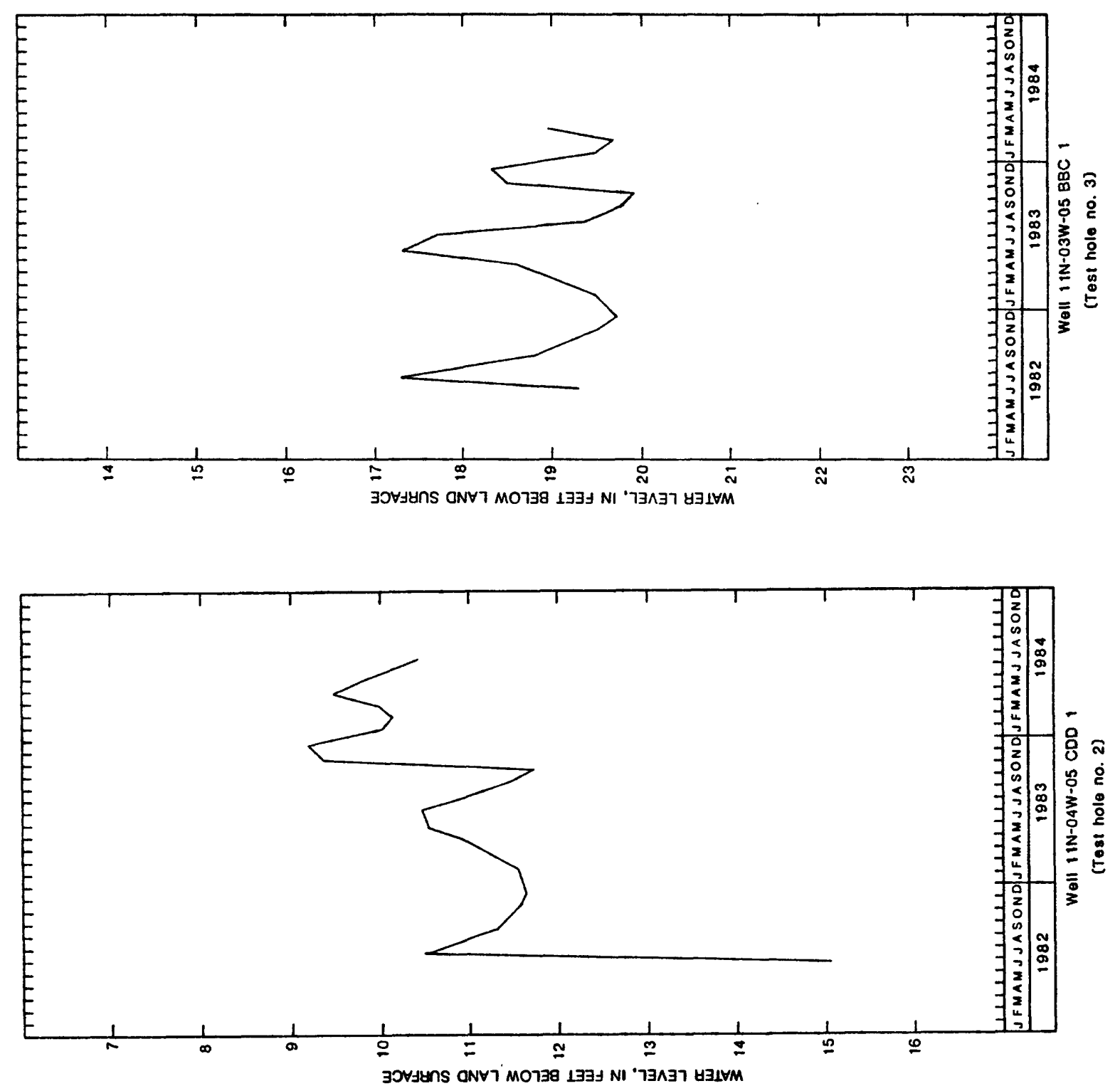

옹

0
1
3
1
2
1
$=$
0
0

5
1
3
0
1
2

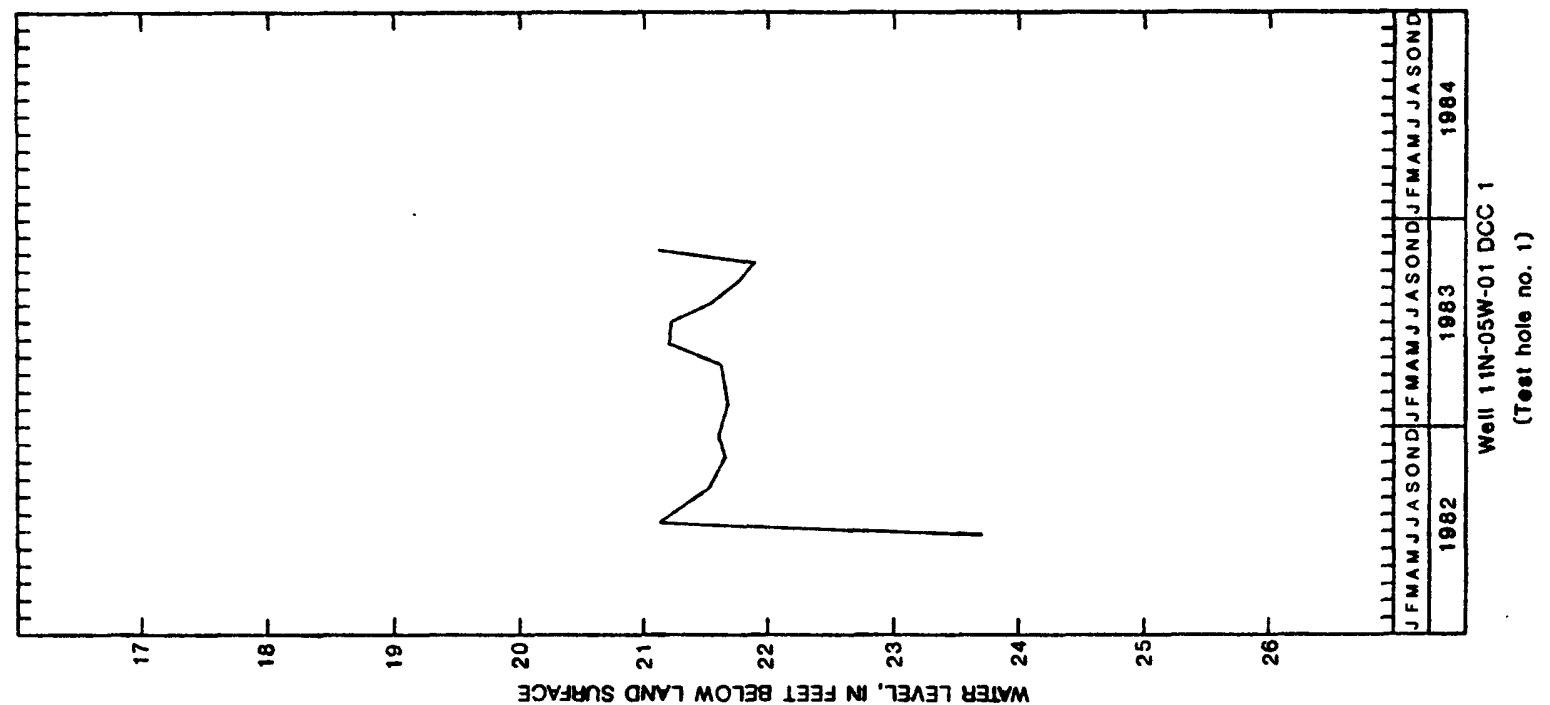

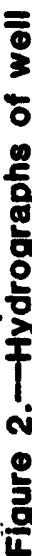




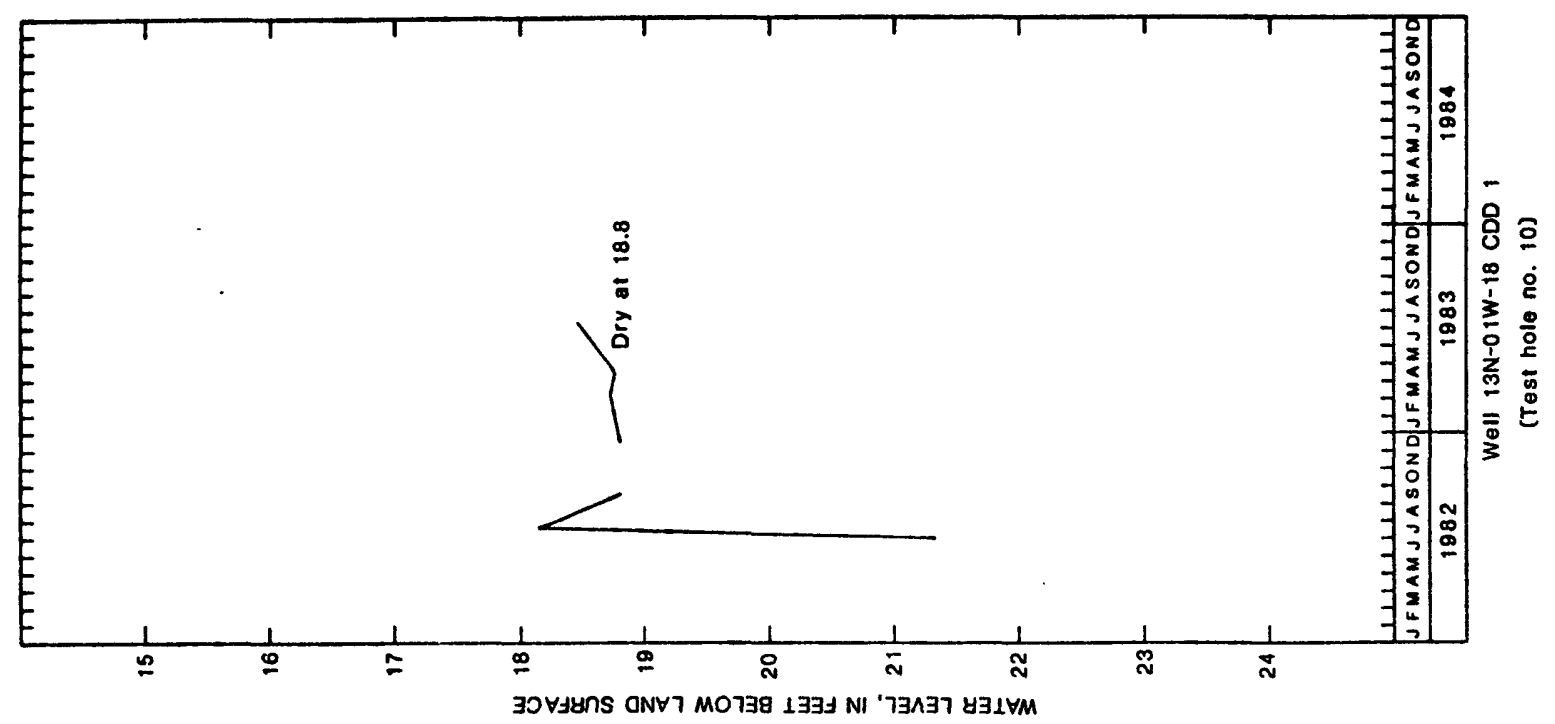

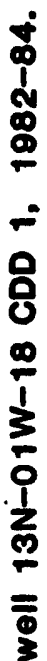
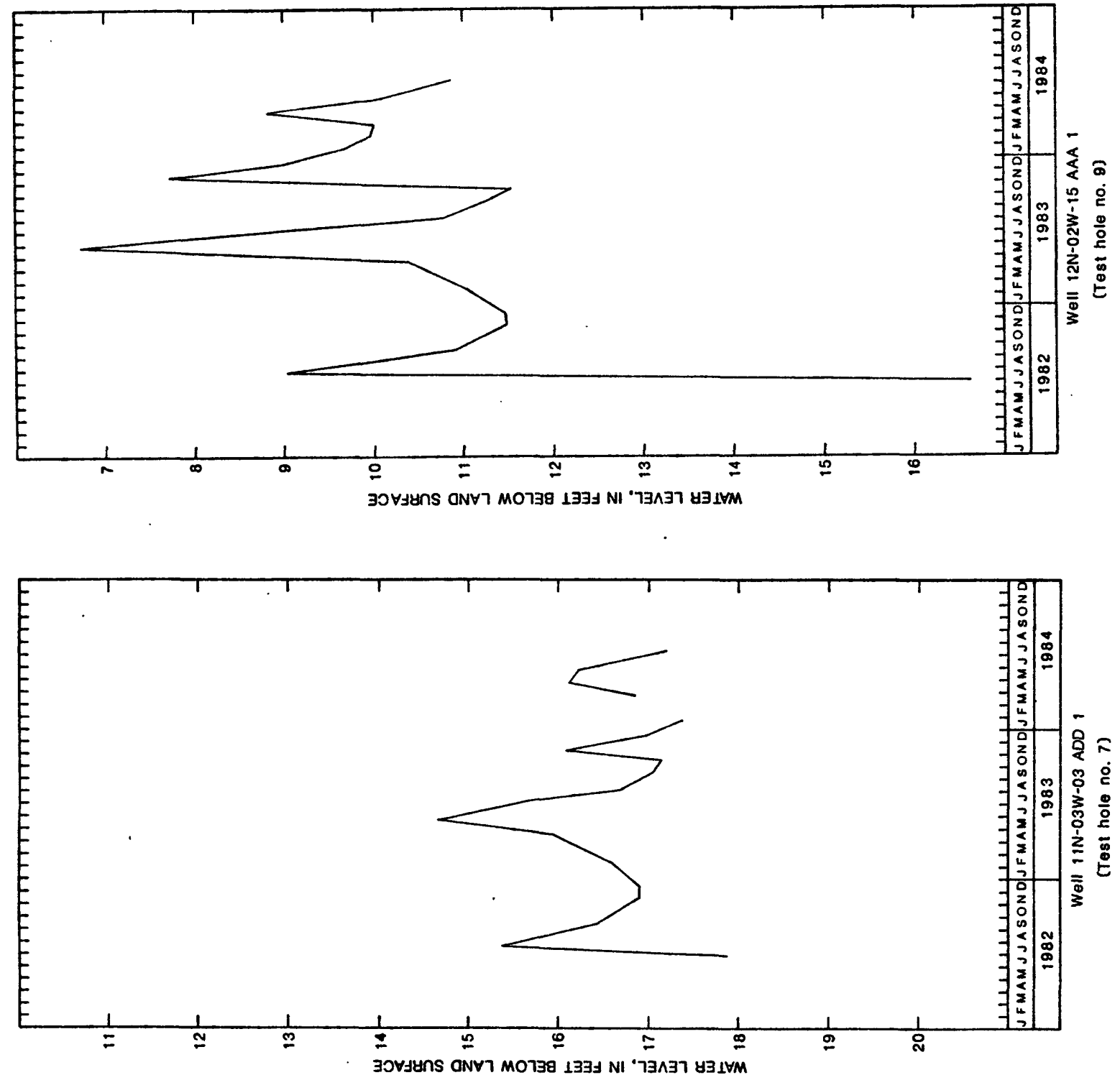

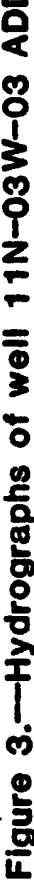



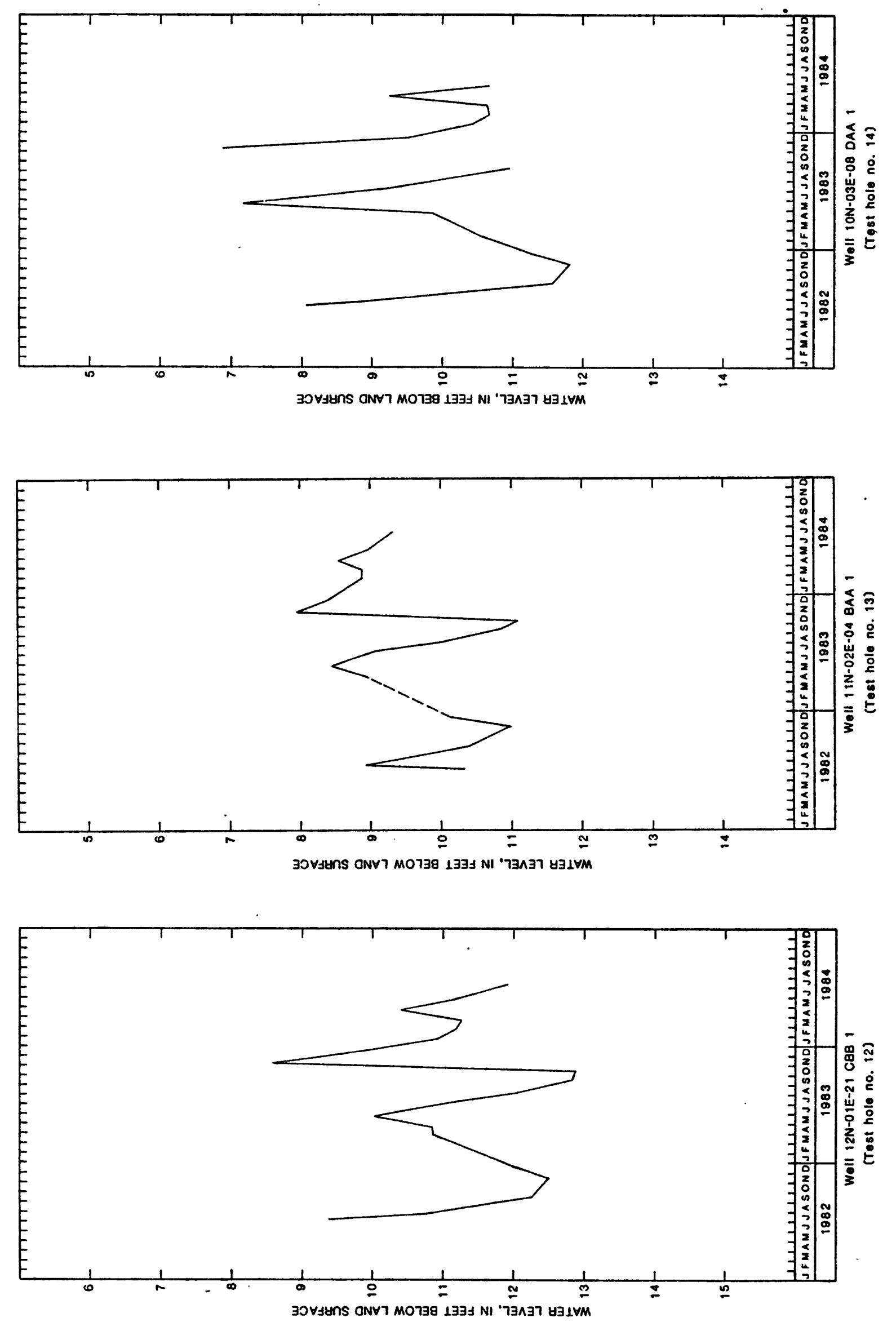

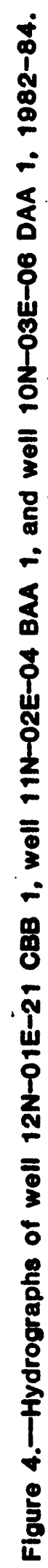




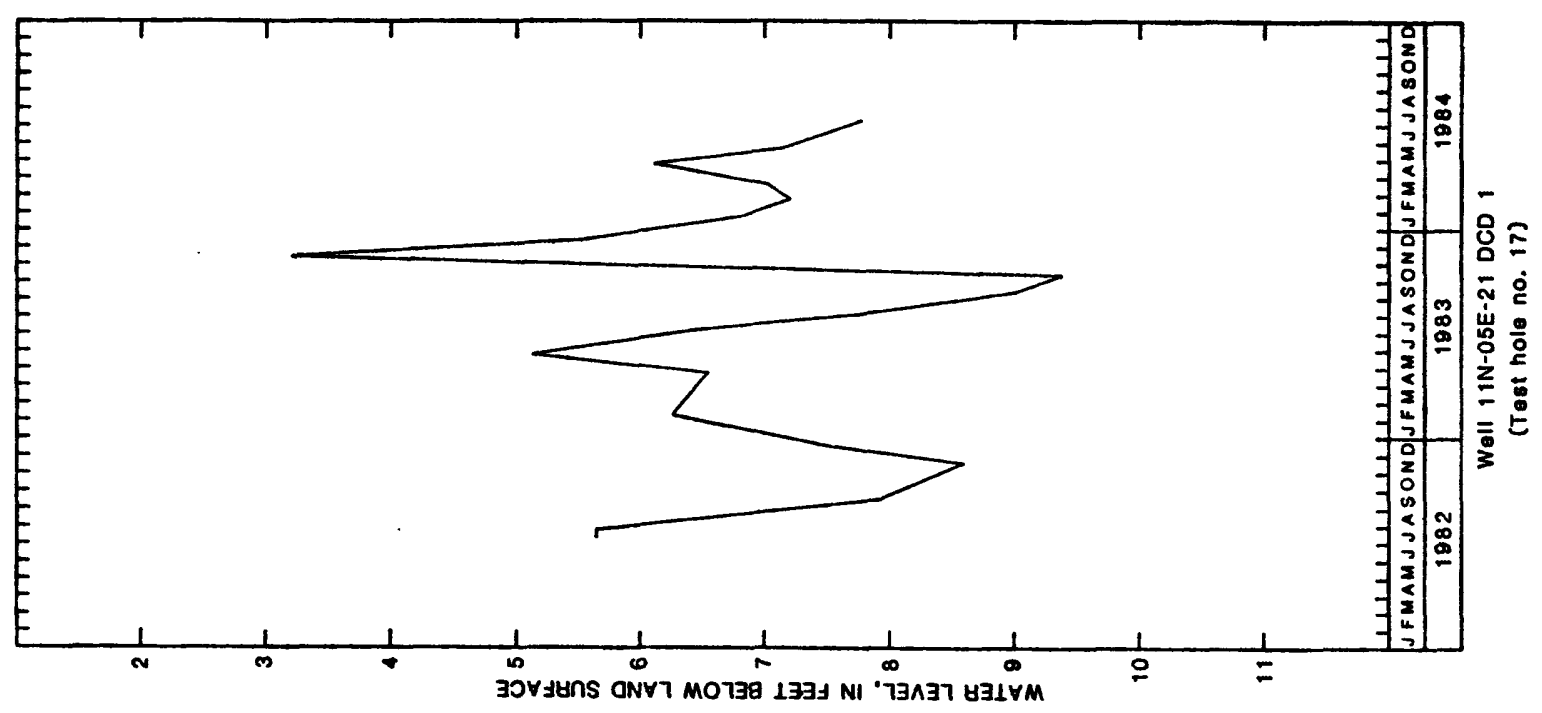

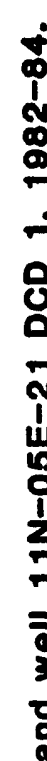
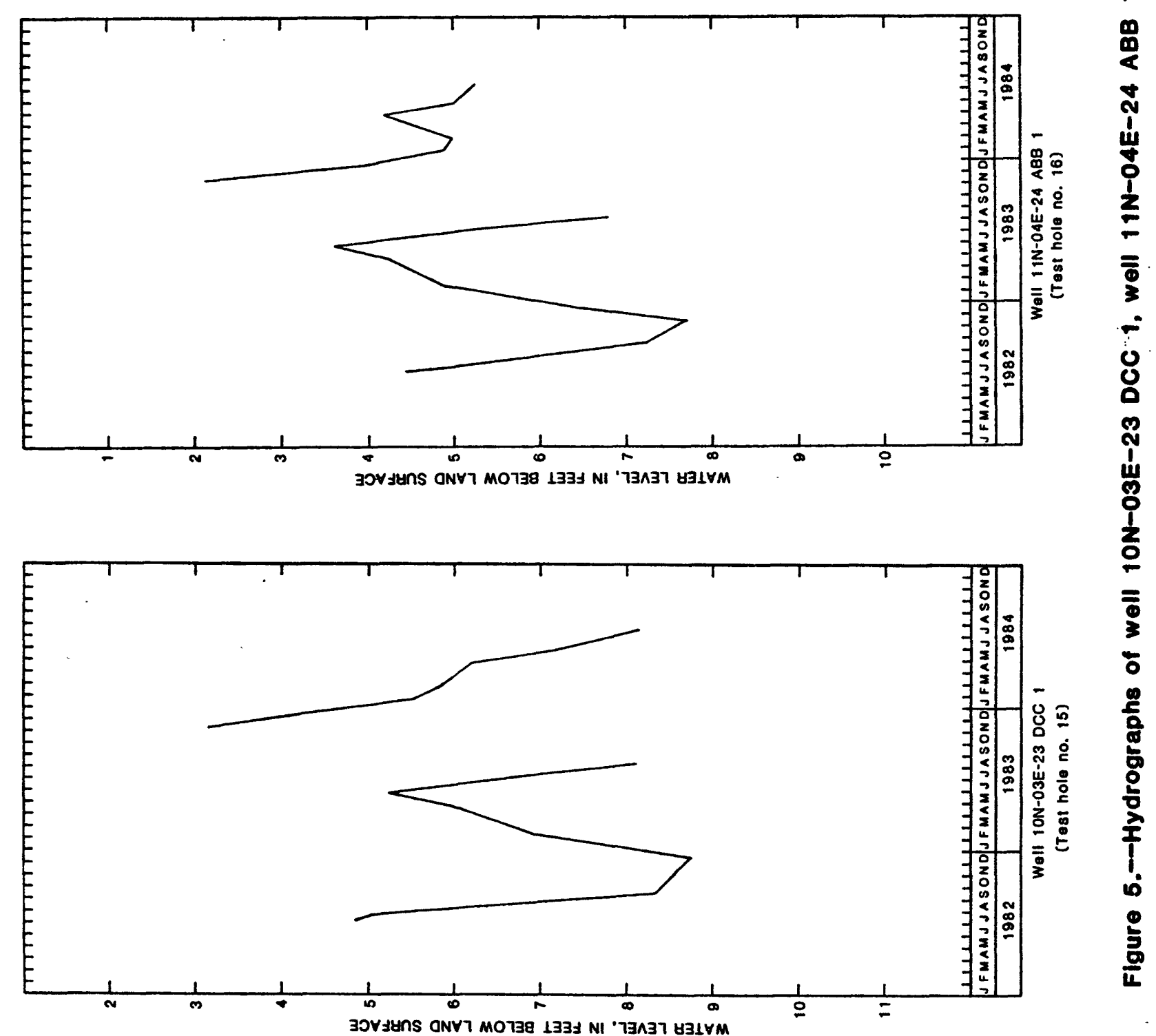


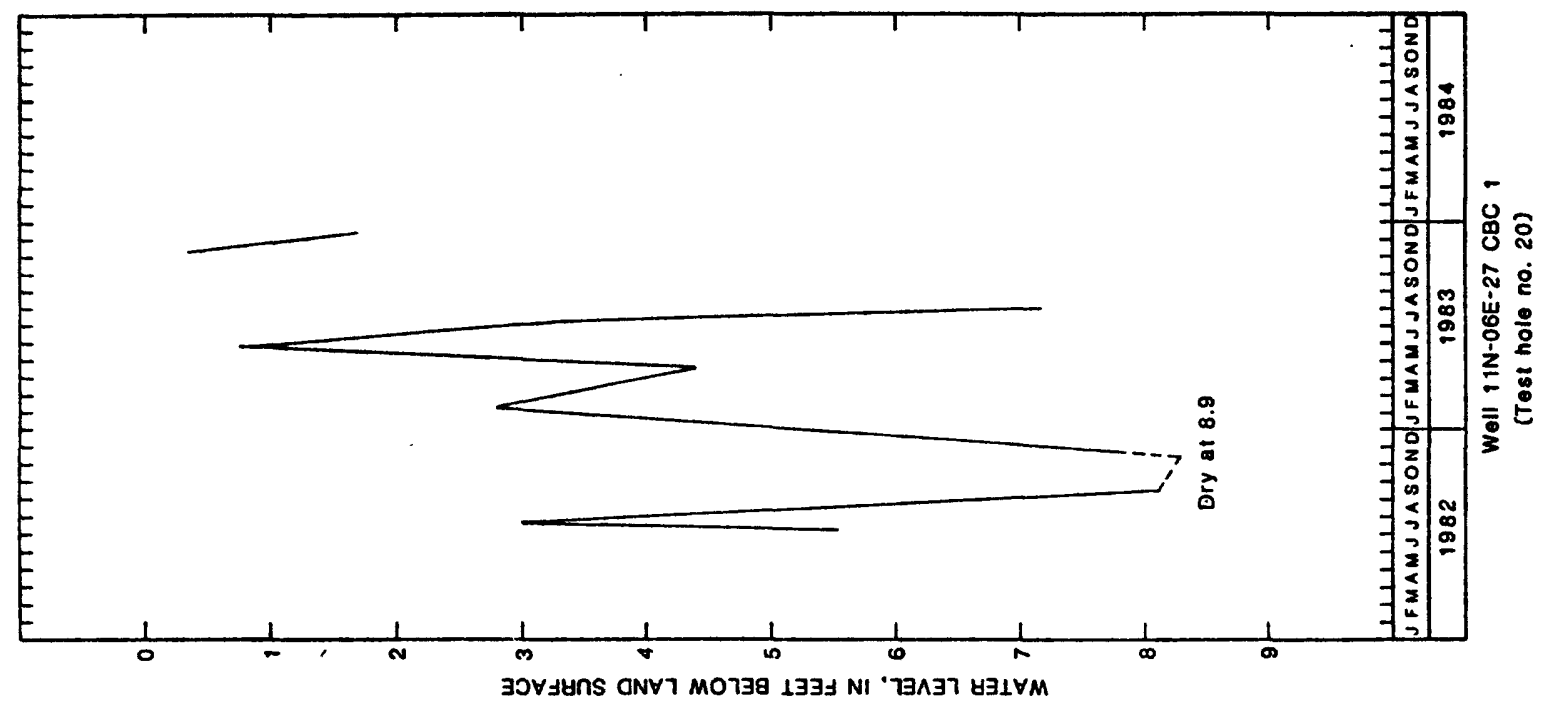

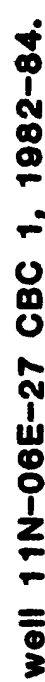
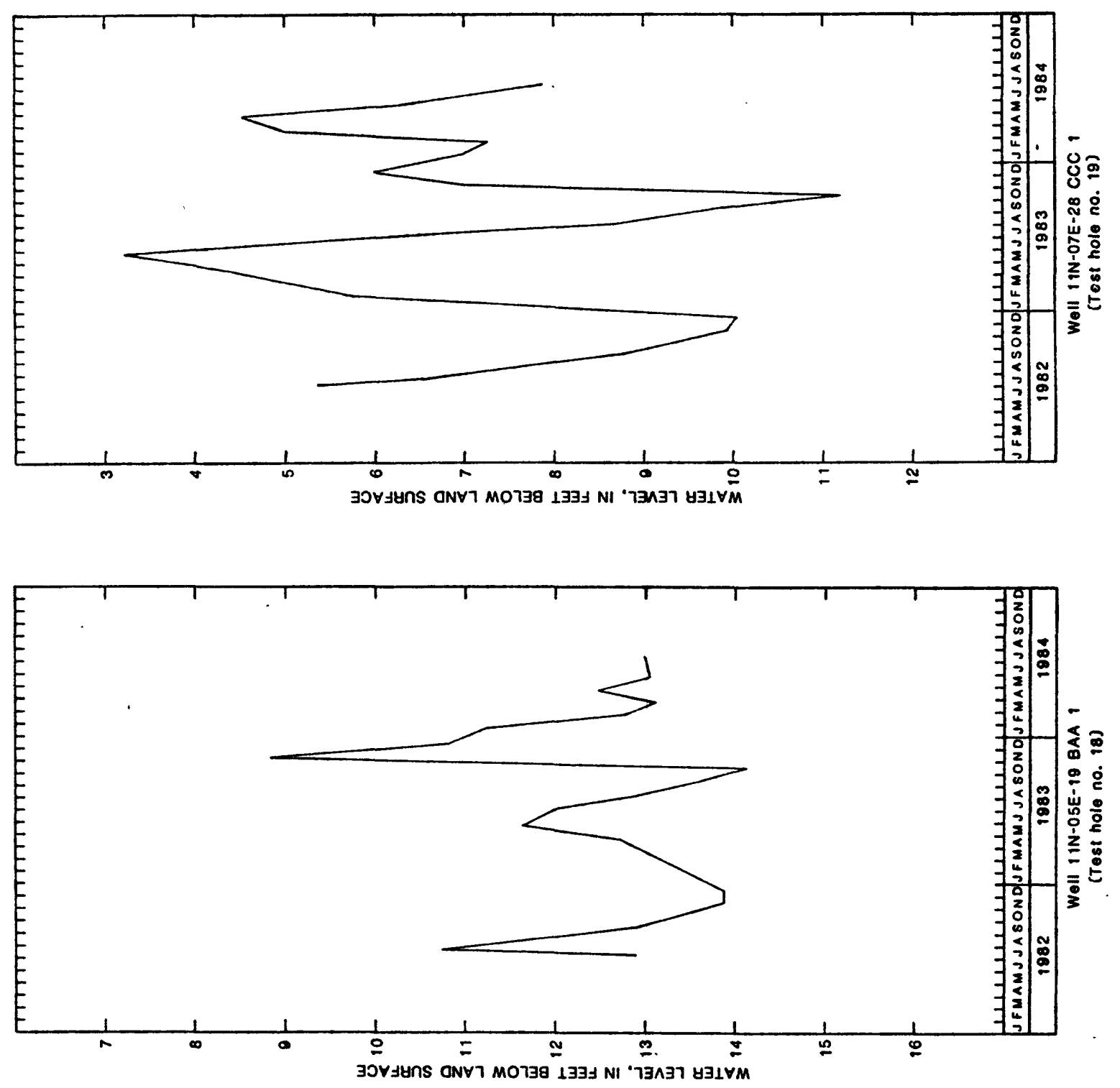

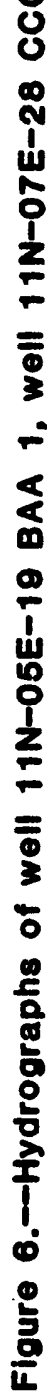



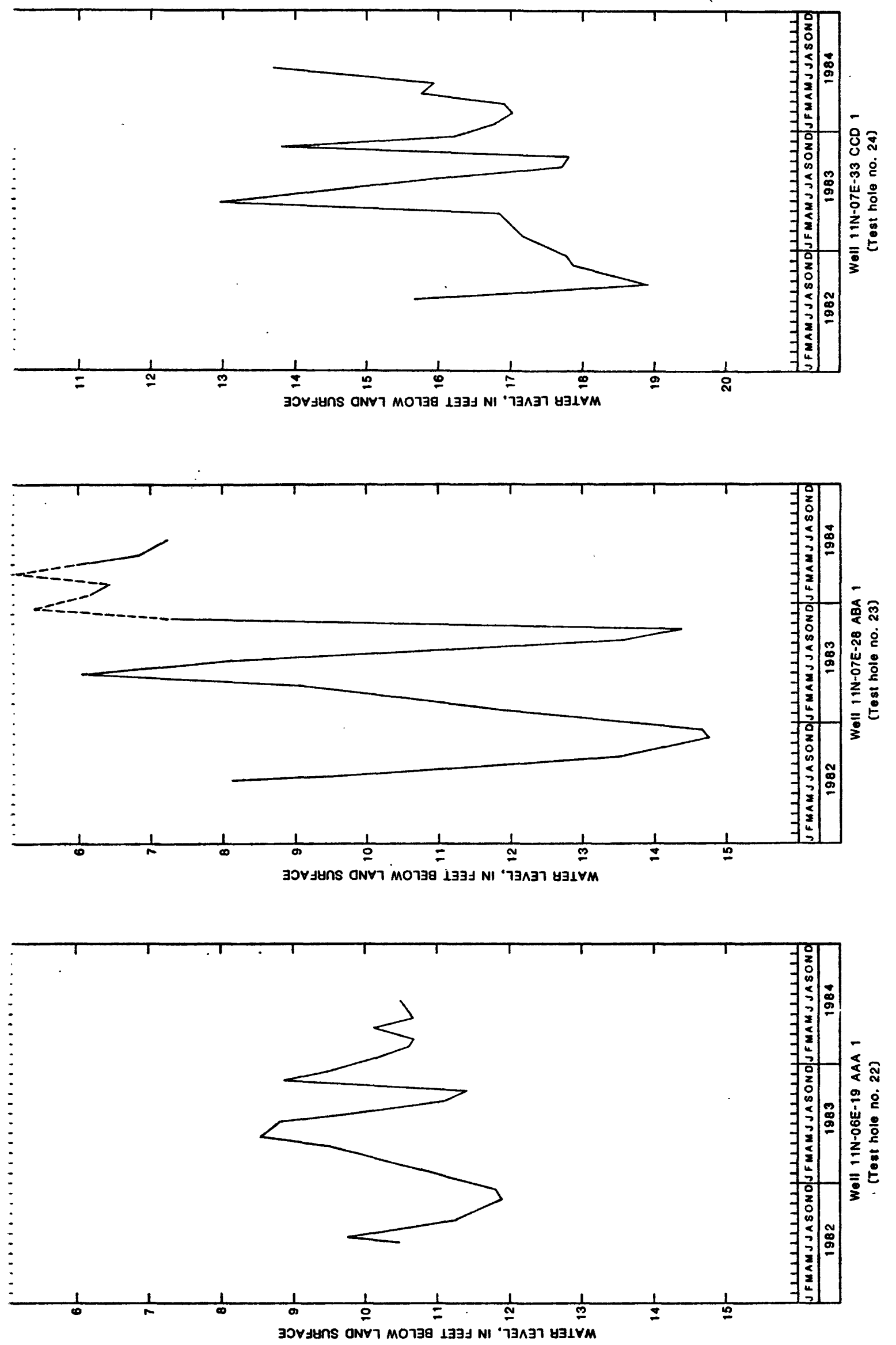

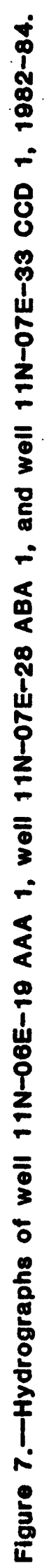



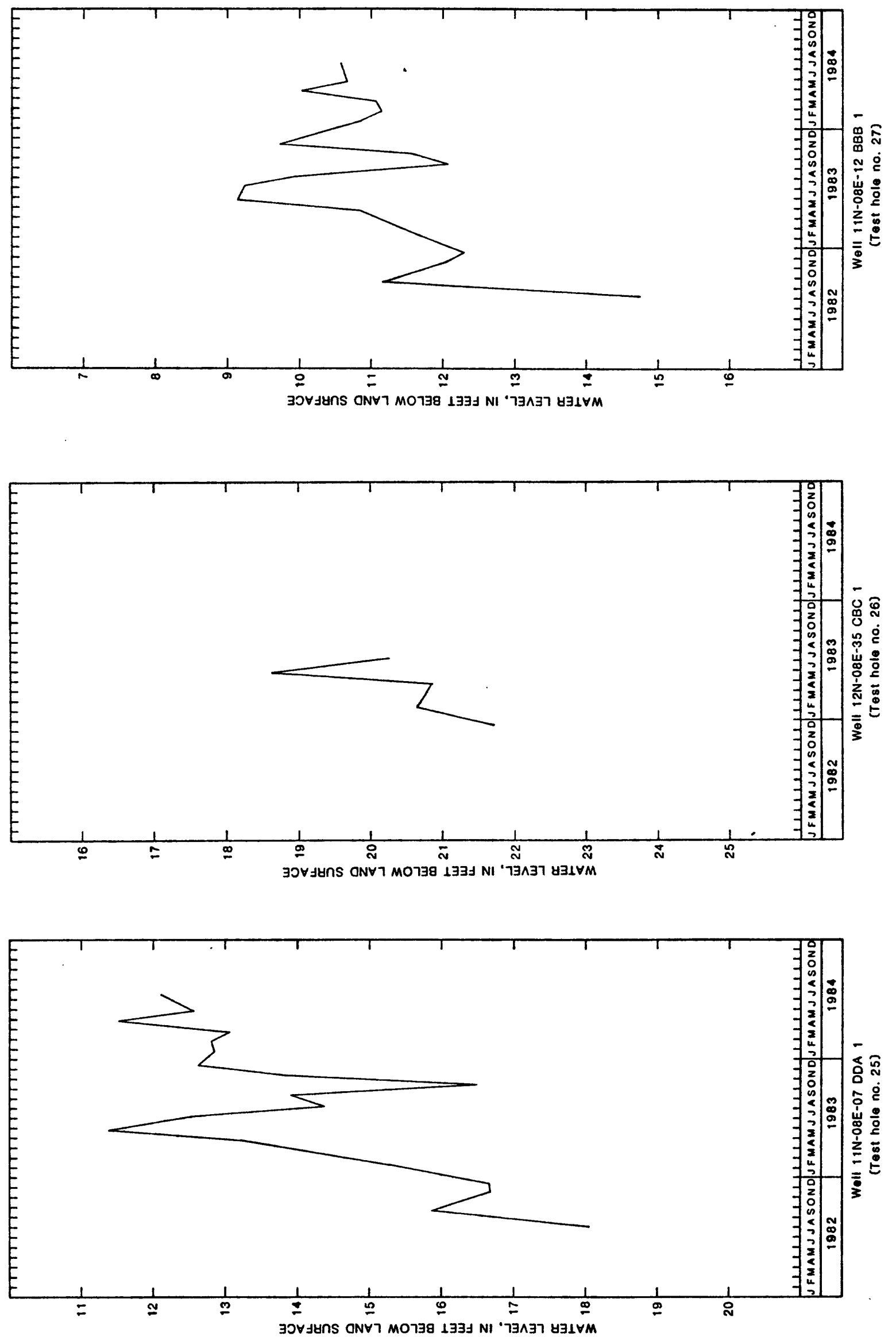

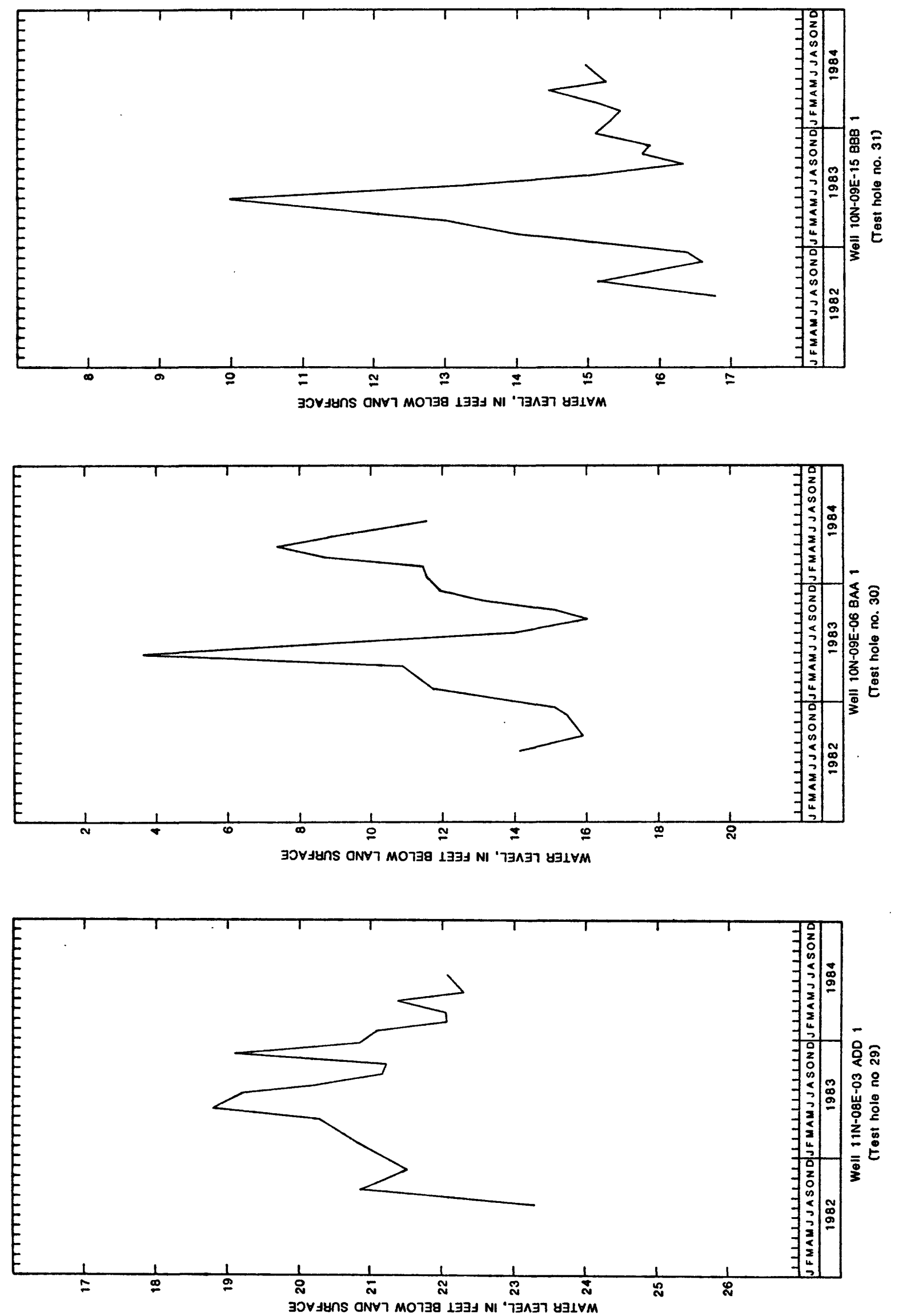

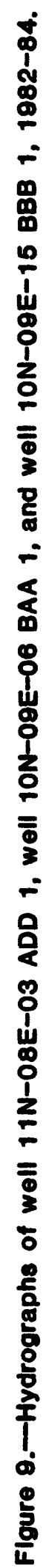




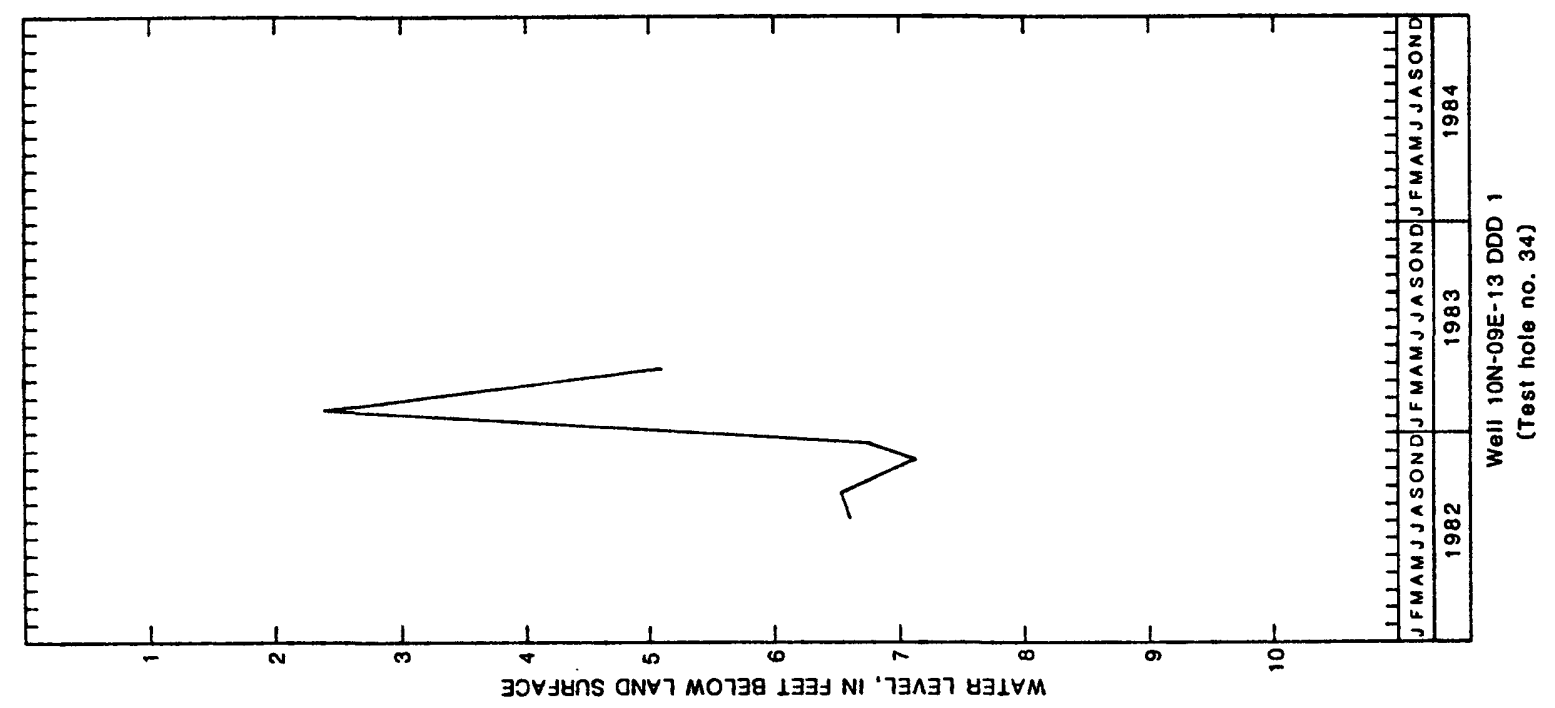

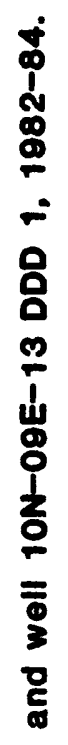
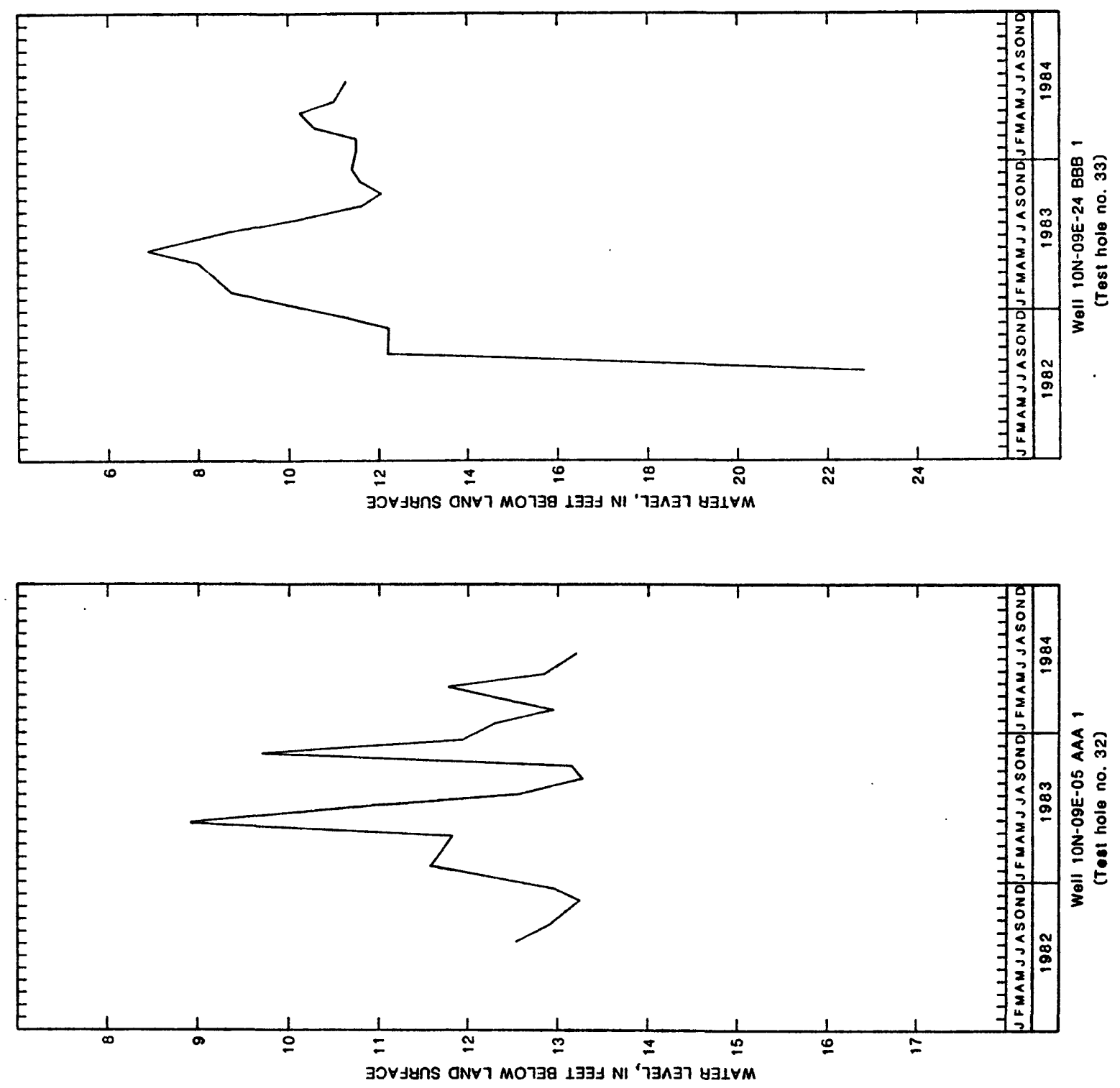

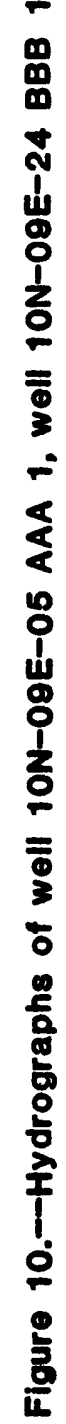



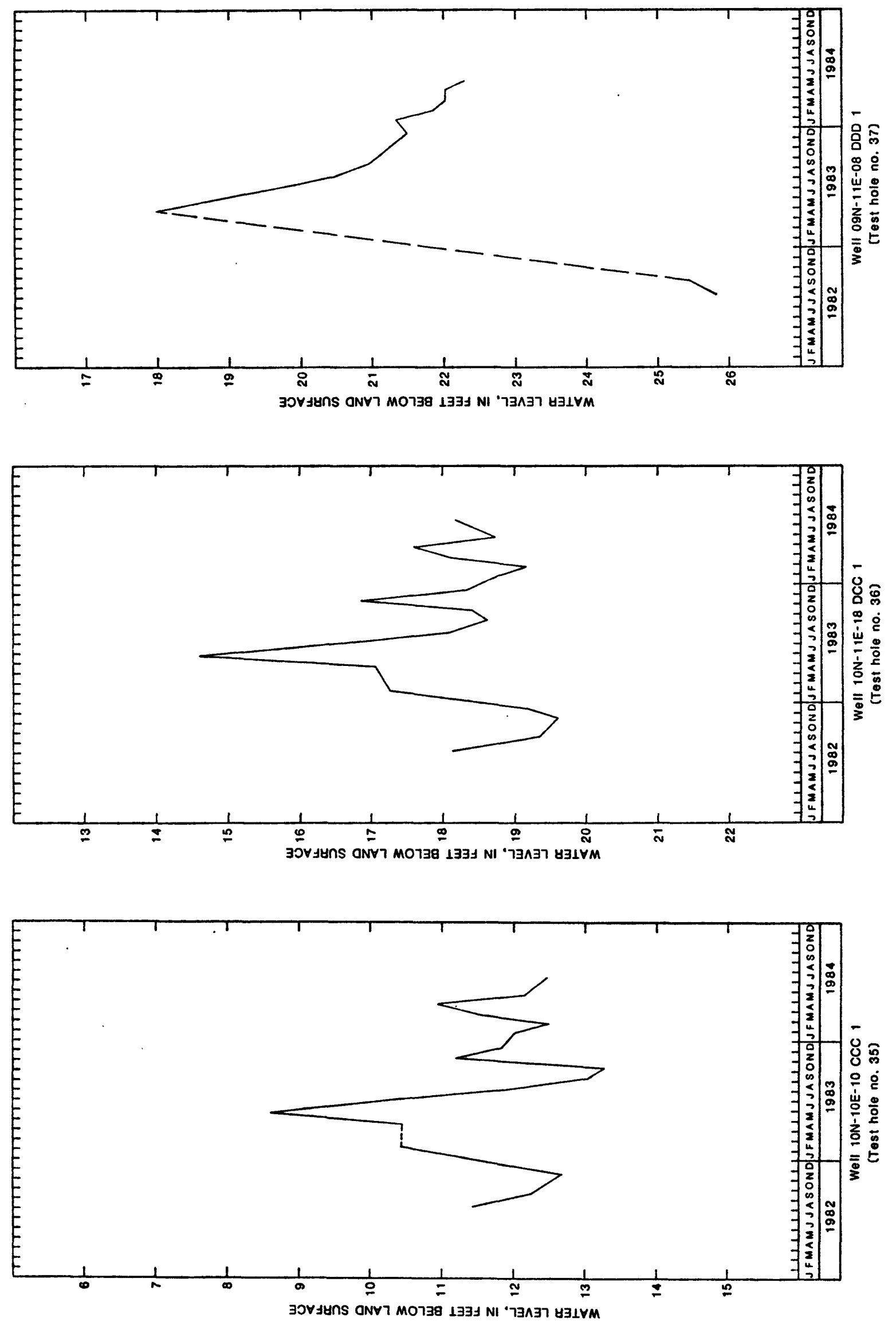

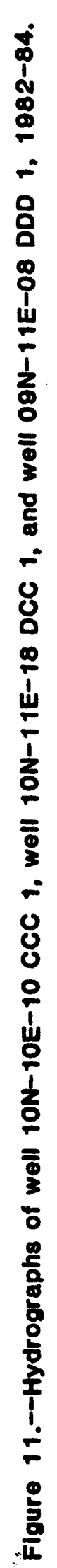




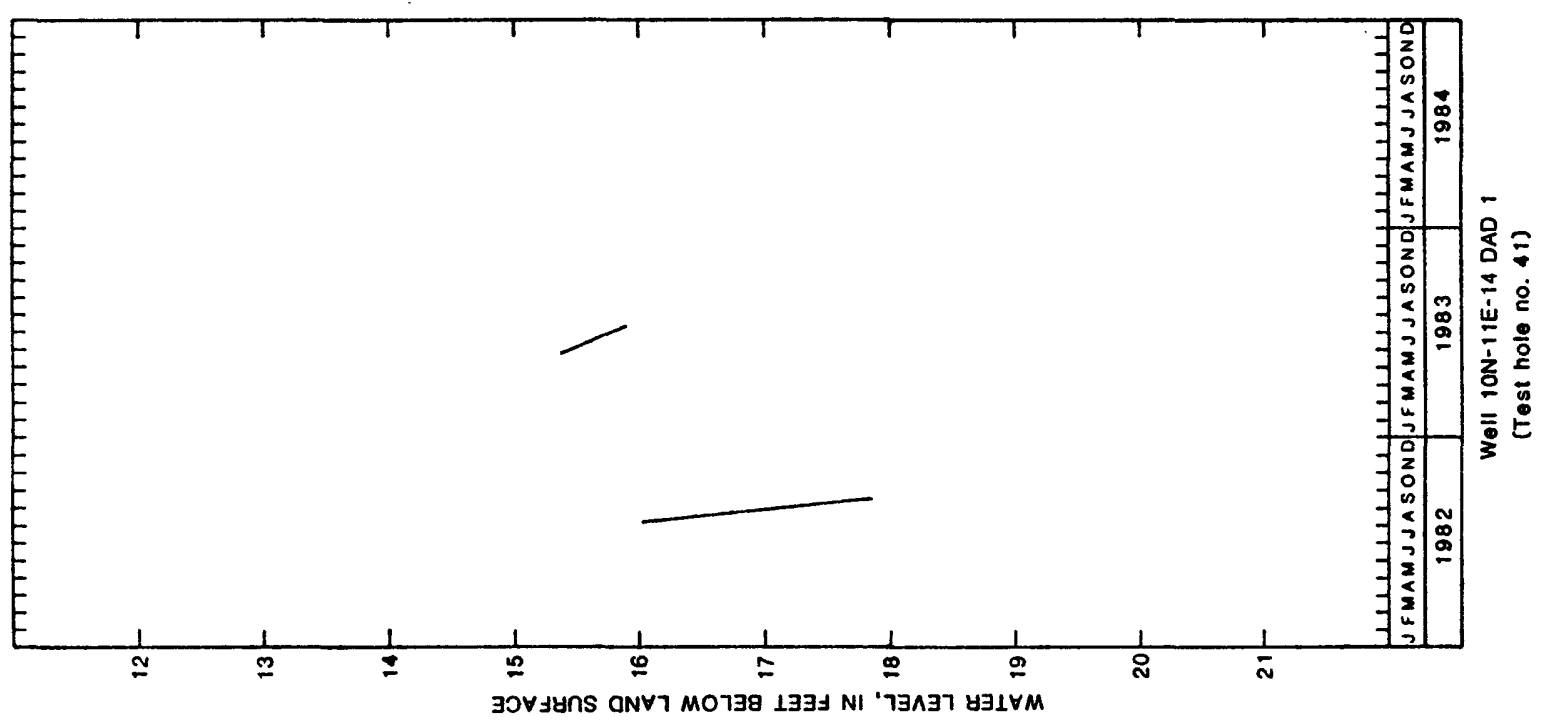

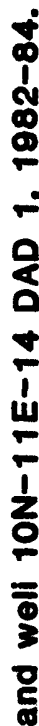
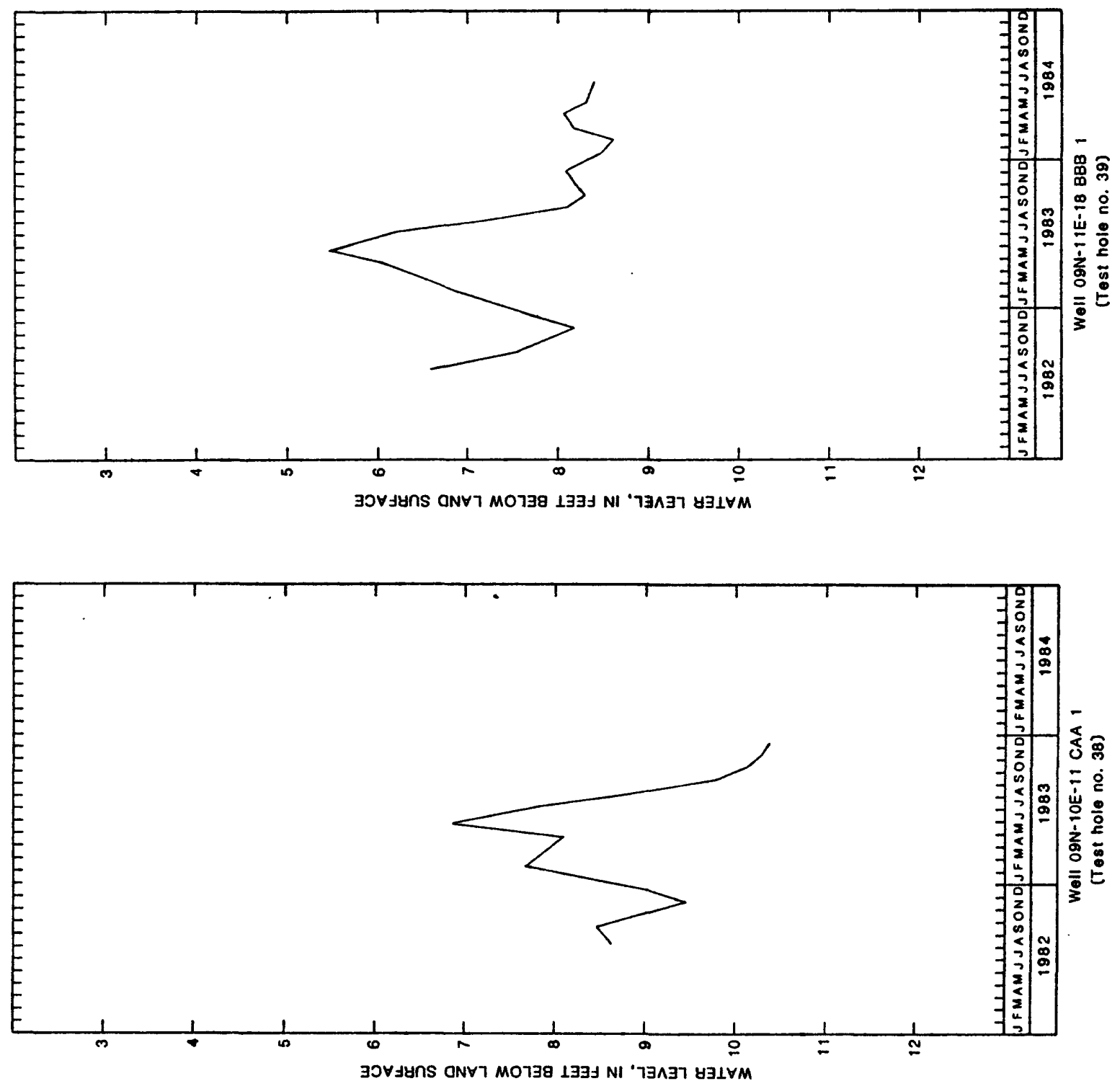

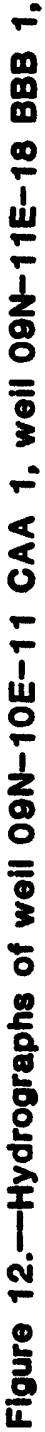



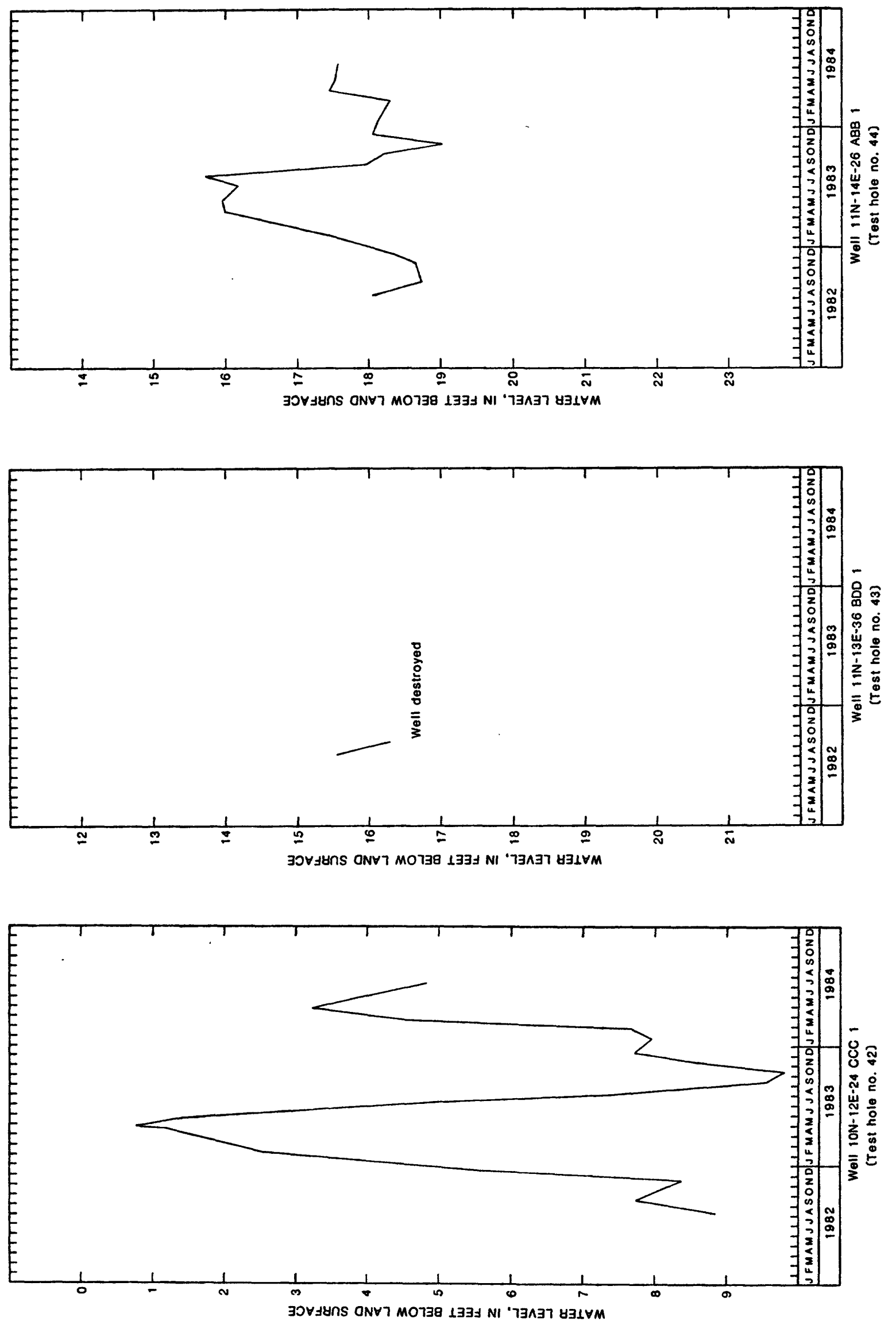

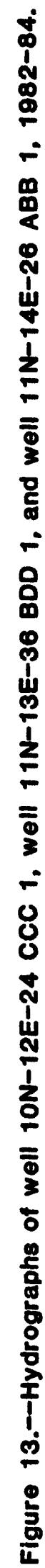




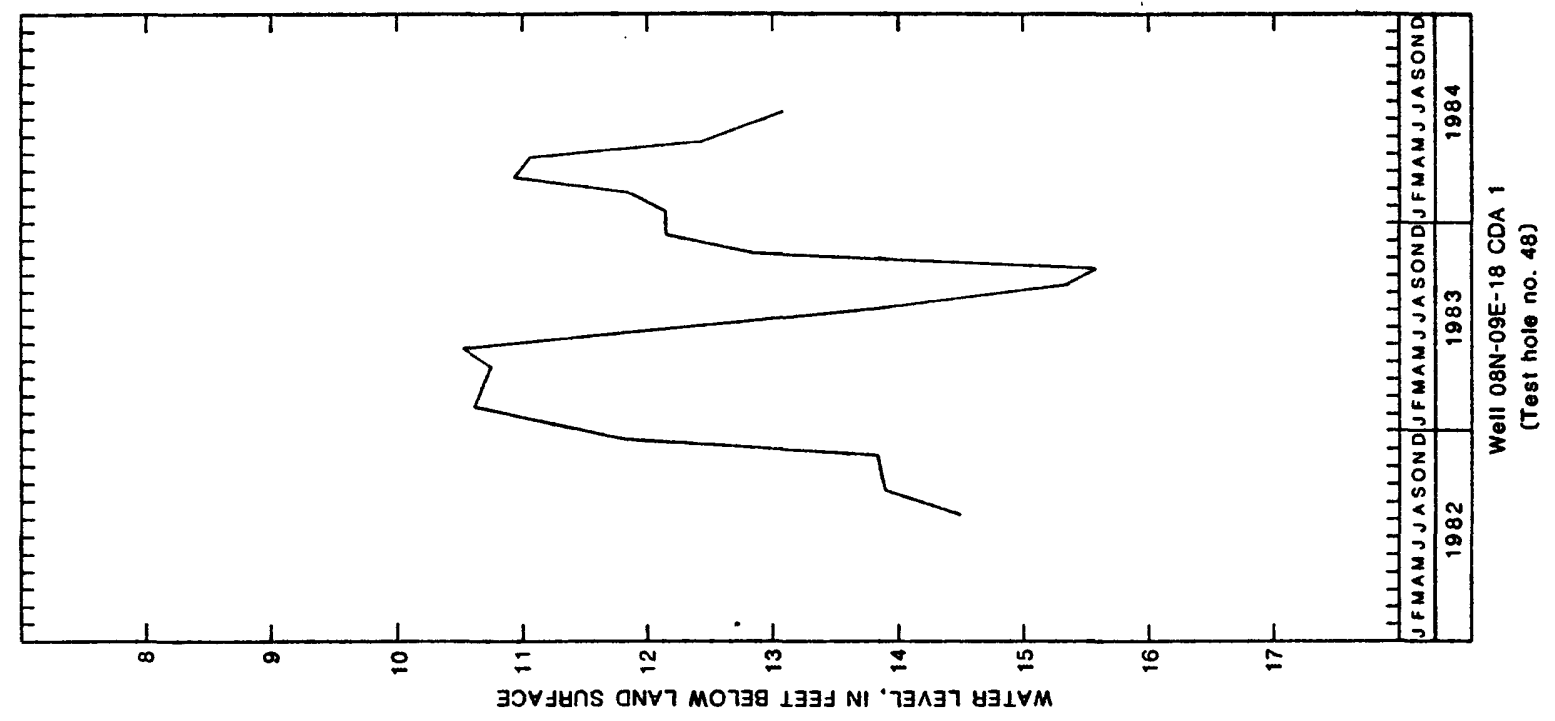

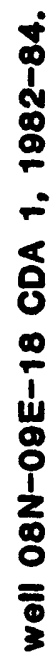
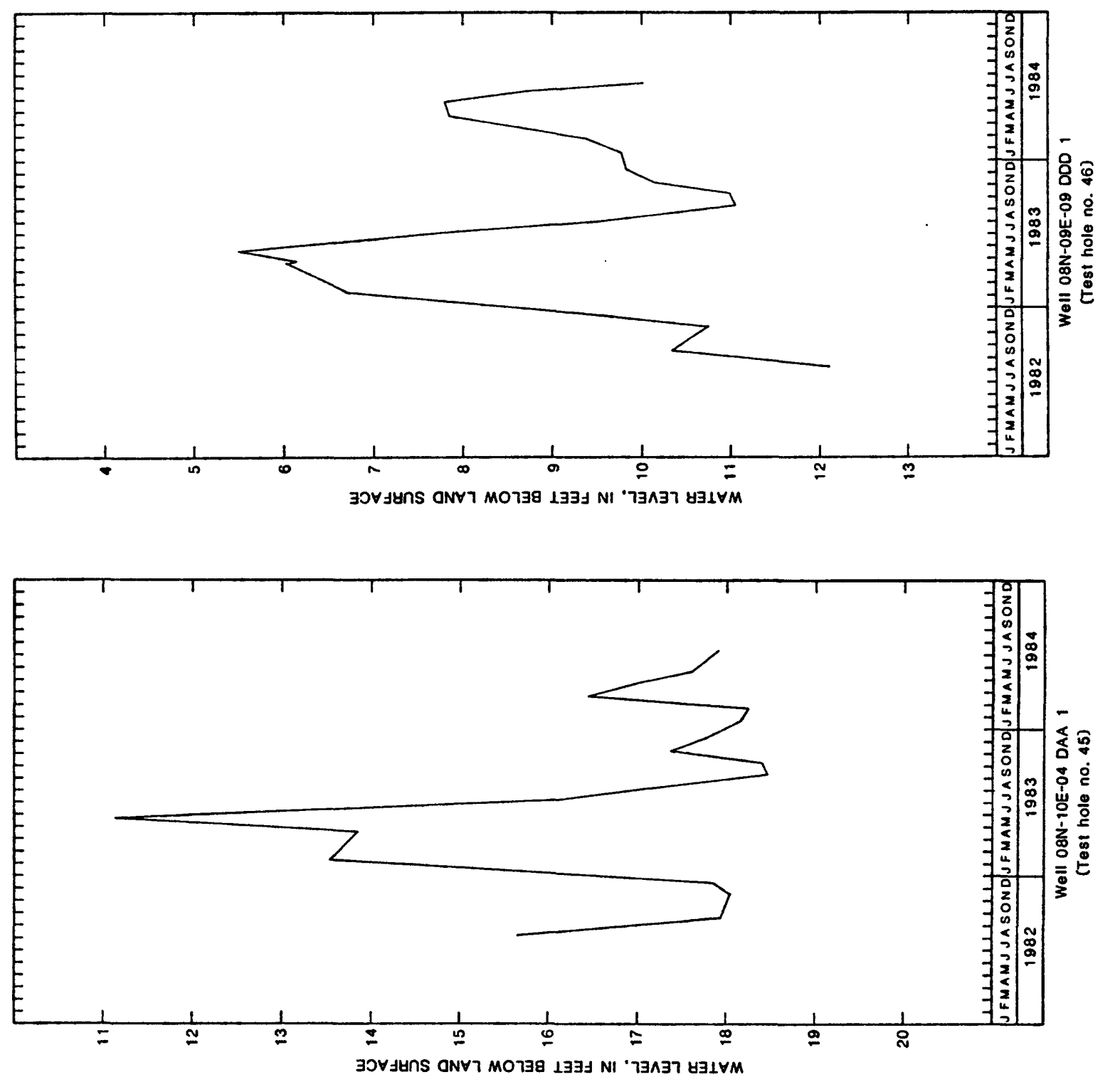

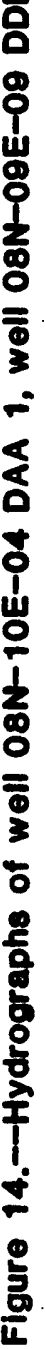




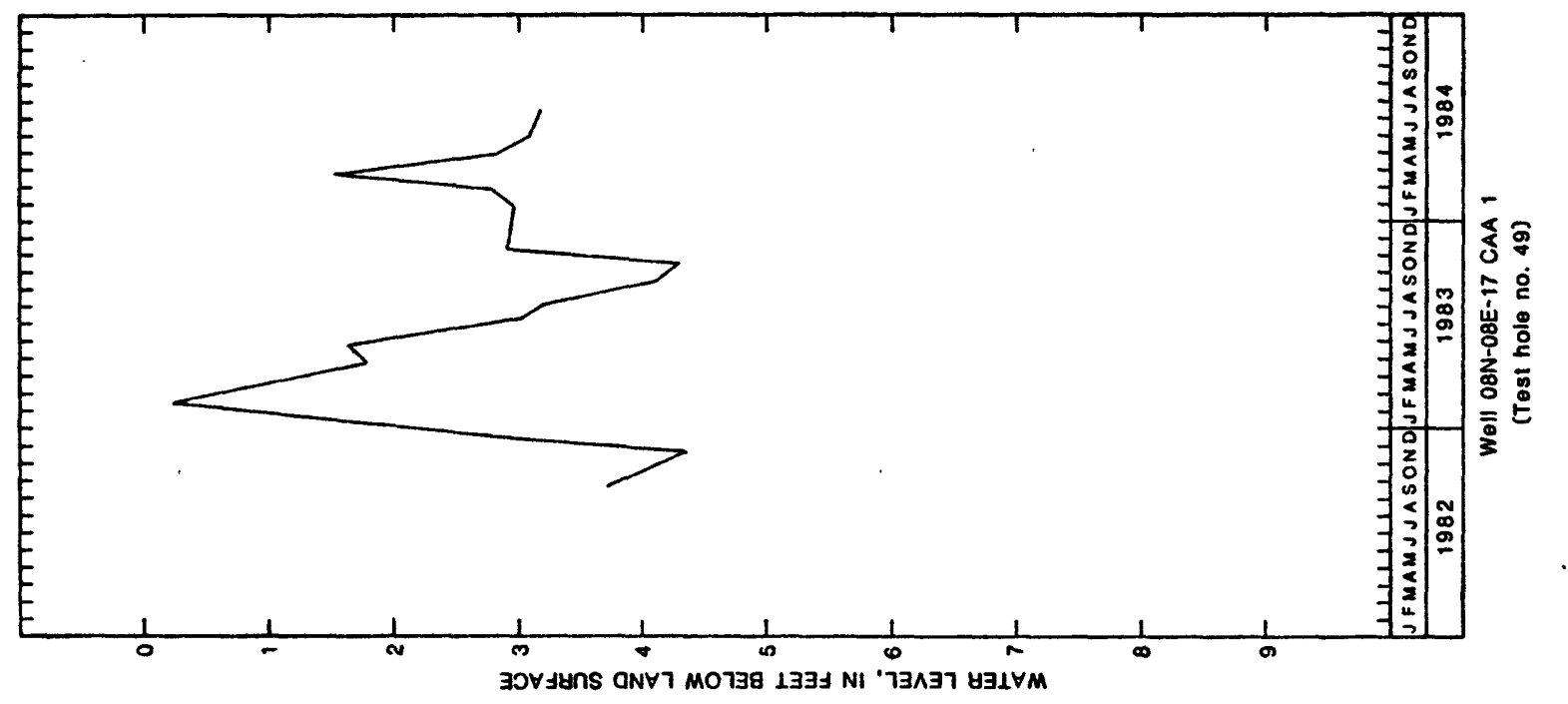

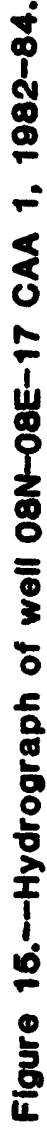




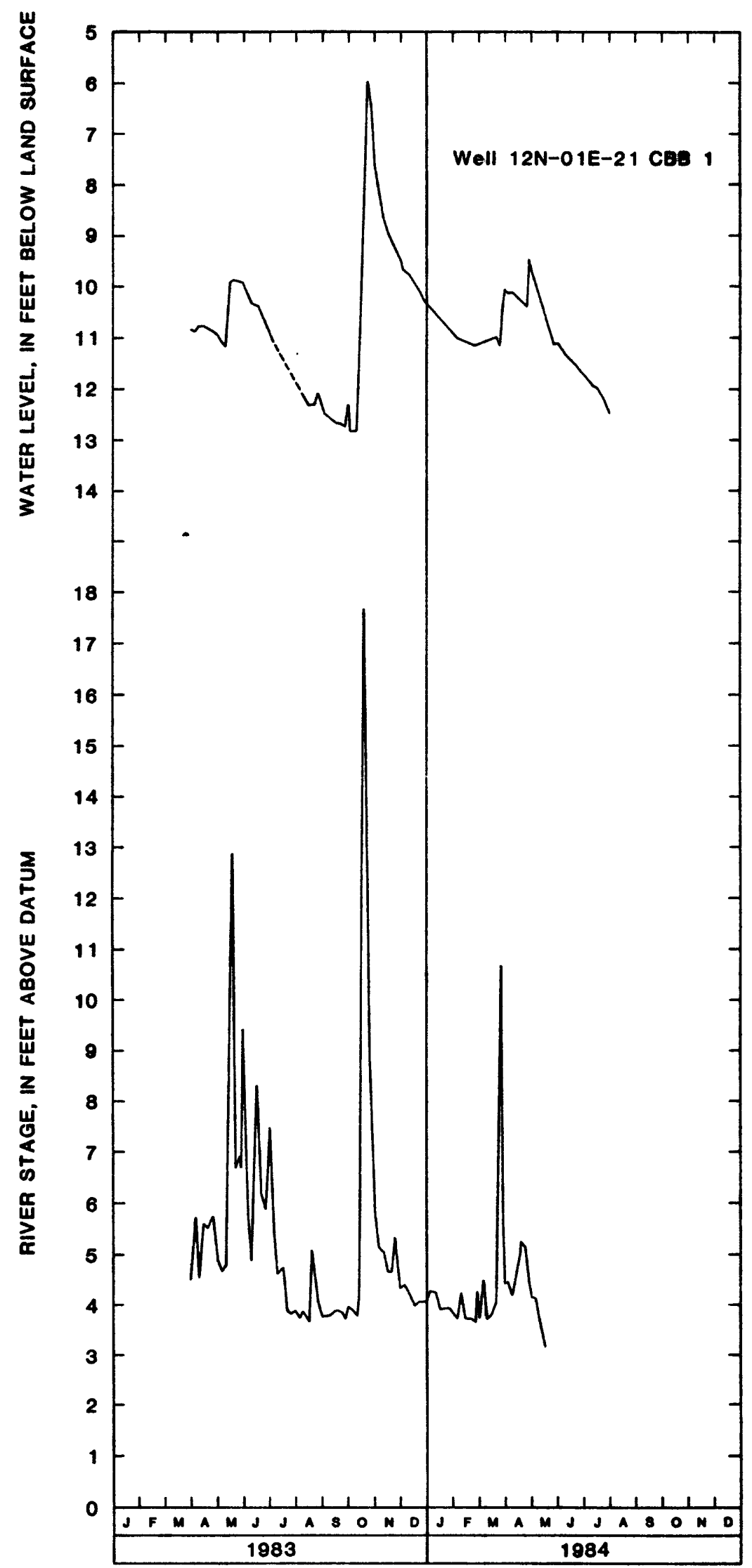

Figure 16.--Hydrographs of well 12N-01E-21 CBB 1 and North Canadian River near Harrah, 1983-84. 


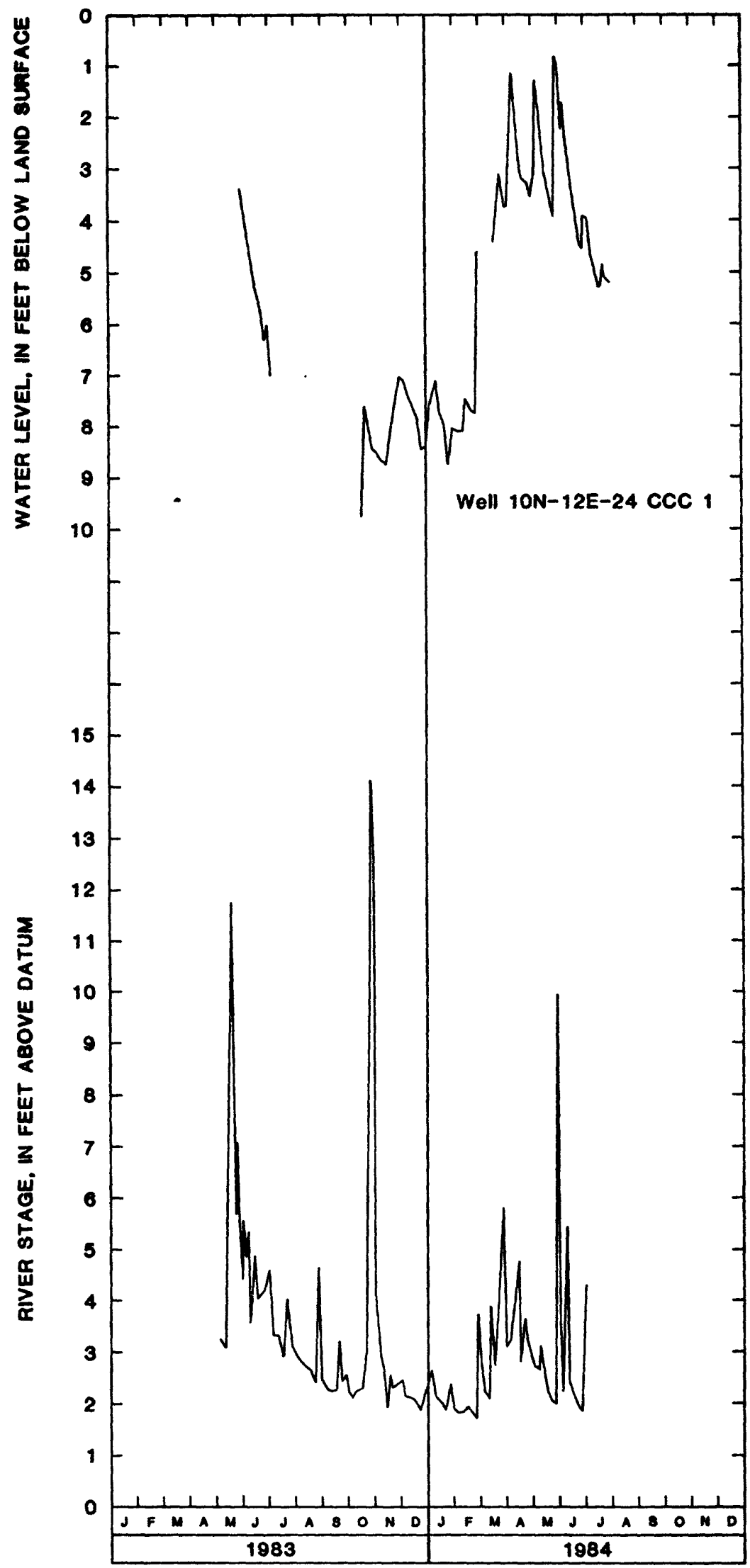

Figure 17.--Hydrographs of well 10N-12E-24 CCC 1 and North Canadian River near Wetumka, 1983-84. 
TABLE 4.--Low-flow measurements of the North Canadian River and tributarles, 1983-84.

8/29-8/31/83
$\begin{gathered}\text { Discharge Difference } \\ \text { (cfs) }\end{gathered}$ (cfs)

North Canadian R. below L. Overholser 9.62

North Canadian R. at I-35 Bridge

Inflow from Oklahoma City sewage

North Canadian R. nr. Harrah

North Canadian R. at Shawnee Bridge

[Squirrel Ck. nr. Shawnee]*

North Canadian R. NE of Shawnee

North Canadian R. nr. Prague

[Turkey Ck. nr. Prague]*

[Gar Ck. nr. Prague]*

[Rock Ck. nr. Paden]*

North Canadian R. W of Okemah

[Flat Rock Ck. nr. Weleetka]*

North Canadian R. nr. Wetumka

[Alabama Ck. at Weleetka]*

[Bad Ck. nr. Graham]*

[Parsley Ck. nr. Graham]*

[Stidham Ck. nr. Graham]*

North Canadian R. above L. Eufaula 18.7

28.3

$2 / 23-2 / 24 / 84$

Discharge Difference (cfs) (cfs)

$$
45.3
$$

18.3

63.6

114

124

$-82$

142

188

$-10$

132

168

2

$$
\text { [0.5]* }
$$

134

$[0.835]^{*}$

191

20

154

194

17

$$
\text { [0.685]* }
$$$$
\text { [2.50]* }
$$$$
\text { [0.665]* }
$$

171

247

$$
-4
$$

167

[0.334]*

314

72

22

$[0.188]^{*}$

$[0.2]^{*}$

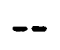

[0.1]* $[0.4]^{*}$

336

Change in flow, Lake Overholser to Lake Eufaula 148

* Tributary which contributes to flow of North Canadian River. Discharge not used in calculation of total flow. 


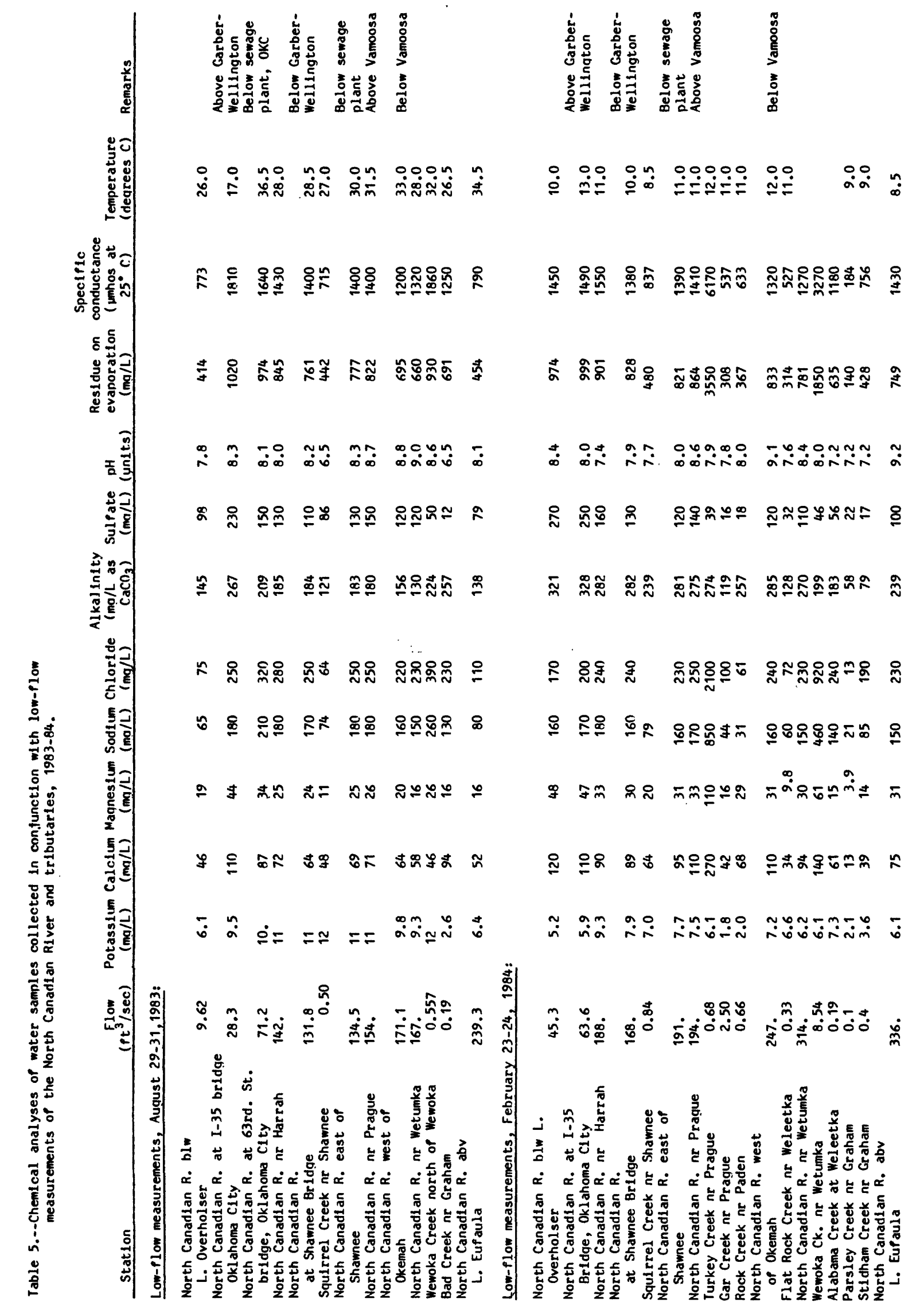

